\title{
PERFORMANCE EVALUATION OF 802.11P VANETS WITH DIFFERENT DUTY CYCLES
}

\author{
by \\ Rishabh Kumar \\ Rajiv Gandhi Technical University \\ Bachelor Of Computer Science and Engineering, 2007 \\ Bhopal, M.P, India

\begin{abstract}
A thesis
presented to Ryerson University

in partial fulfillment of the

requirements for the degree of

Master of Science

In the Program of

Computer Science
\end{abstract}

Toronto, Canada, 2011

(C) Rishabh Kumar 2011 


\section{Author's Declaration}

I hereby declare that I am the sole author of this thesis.

I authorize Ryerson University to lend this thesis to other institutions or individuals for the purpose of scholarly research.

Signed:

I further authorize Ryerson University to reproduce this thesis by photocopying or by other means, in total or in part, at the request of other institutions or individuals for the purpose of scholarly research.

Signed: 


\title{
Performance Evaluation of 802.11P VANETs With Different Duty CyCles
}

\author{
Rishabh Kumar \\ M. Sc. in Computer Science, \\ Ryerson University, Toronto, Canada
}

\begin{abstract}
With the improvement in technology, the number of vehicles on the roads has increased tremendously over the last decade. As large number of vehicles are on the road it has become almost a necessity to improve the driving conditions, provide all the facilities and to make sure that driving is safe for the drivers around the world. To utilize this information the vehicle has to alternately switch between control $(\mathrm{CCH})$ and service channels $(\mathrm{SCH})$ effectively. So, in our work we evaluate the performance of the vehicles on control and service channels with different duty cycle such that no critical message is missed and multimedia applications can be used concurrently. For this purpose we developed 802.11p simulator and the experiments proved that $\mathrm{CCH}$ should use duty cycle of 0.6 as delay tolerant data has to be transmitted over it and SCH should work on 0.3 which is delay insensitive, but generates revenue for the industry by using different multimedia applications as requested by the user.
\end{abstract}




\section{Acknowledgements}

I would like to thank the Almighty Lord for always being with me.

I would like to thank my supervisor Dr. Jelena Misic who supported me throughout my Masters degree and introduced me to the field of vehicular networks. With her guidance and support I could carry on this research. A special thanks for Dr. Vojislav Misic who introduced me to the Artifex and for his patience and guidance during this period.

Thanks for all the support from my family. 


\section{Dedication}

To my family 


\section{Table of Contents}

1 Introduction $\quad 1$

1.1 VANET Characteristics . . . . . . . . . . . . . . . . . . 2

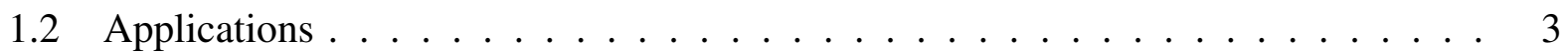

1.3 Problem Statement . . . . . . . . . . . . . . . . . . 5

1.4 Solution Approach . . . . . . . . . . . . . . . . . 5

1.5 Thesis Contribution . . . . . . . . . . . . . . . . . . . 6

1.6 Thesis Organization . . . . . . . . . . . . . . . 6

2 Background and Related Work $\quad 7$

2.1 Distributed Coordination Function . . . . . . . . . . . . . . . . 7

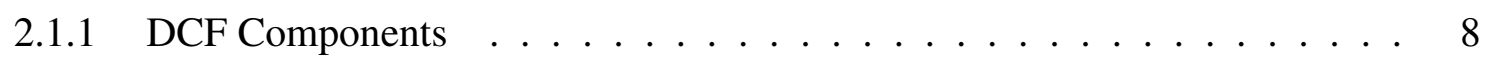

2.1.2 Basic Access Mechanism . . . . . . . . . . . . . . . . . . 9

2.1.3 Hidden Node Problem . . . . . . . . . . . . . . . . . . . . 11

2.1 .4 RTS/CTS Scheme . . . . . . . . . . . . . . . 12

2.1 .5 CSMA/CA for DCF . . . . . . . . . . . . . . 13

2.1 .6 Limitations of DCF . . . . . . . . . . . . . . . . . 14

2.1 .7 Point Coordinated Function . . . . . . . . . . . . . . . . 14

2.1 .8 Limitations of $\mathrm{PCF} \ldots \ldots \ldots \ldots \ldots$

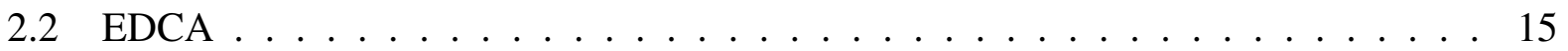

2.2.1 Interframe Space Relationship . . . . . . . . . . . . . . . 16

2.2 .2 EDCA Parameters . . . . . . . . . . . . . . . . 18

2.3 Description of IEEE $802.11 \mathrm{p}$ standard . . . . . . . . . . . . . . . . . . 21

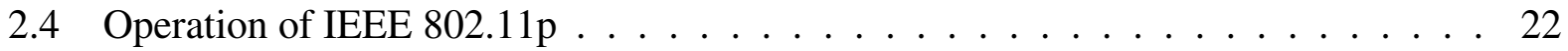

3 Simulator Design $\quad 29$

3.1 Block Diagram of Simulator . . . . . . . . . . . . . . . . . 30

3.2 Top Level . . . . . . . . . . . . . . . . . . . . . . . . . . 31

3.3 Measurement . . . . . . . . . . . . . . . . . 32

3.4 Road Side Unit . . . . . . . . . . . . . . . . . . . . . . . . . . . 34

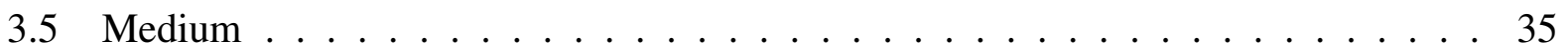

3.6 Device . . . . . . . . . . . . . . . . . . 37

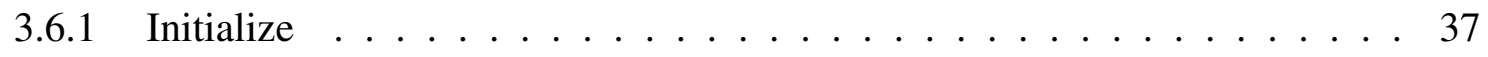

3.6 .2 Source Poisson . . . . . . . . . . . . . . . . . 39

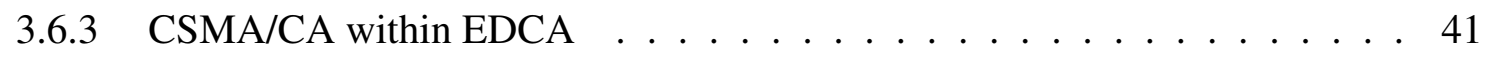

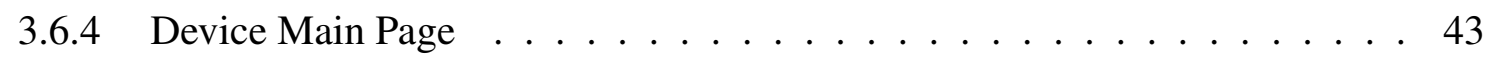

4 Performance Evaluation and Analysis of Results 45

4.1 Simulation Parameters . . . . . . . . . . . . . . . . . 45 
4.2 Simulation Scenarios and Performance Evaluation of 802.11 DCF . . . . . . . . 47

4.3 Performance Evaluation of 802.11e EDCA . . . . . . . . . . . . . . . . 56

4.4 Performance Evaluation and Analysis of Results for 802.11p With Different Duty Cycles . . . . . . . . . . . . . . . . . . . . . 58

4.5 High Data Rate Experiment . . . . . . . . . . . . . . . . . . . . . 74

5 Conclusion 


\section{List of Tables}

2.1 Access Category Used In Our Model. . . . . . . . . . . . . . . . . . . . 16

2.2 Default Values of EDCA Parameters. . . . . . . . . . . . . . . . . . 20

2.3 CCH Parameters. . . . . . . . . . . . . . . . . . . . . . . . . . . . . . . . . . . . . . .

2.4 SCH Parameters. . . . . . . . . . . . . . . . . . . . . 26

4.1 Node Population. . . . . . . . . . . . . . . . . . . . . . . . 45

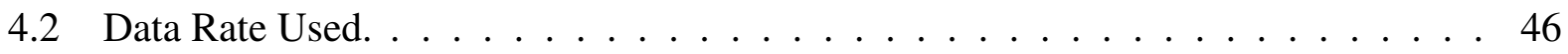

4.3 Legend Notations. . . . . . . . . . . . . . . . . . . . . . 46

4.4 New Data Rate Used . . . . . . . . . . . . . . . . . . . . 75 


\section{List of Figures}

$1.1 \quad$ VANETs Scenario . . . . . . . . . . . . . . . . . . . . . 1

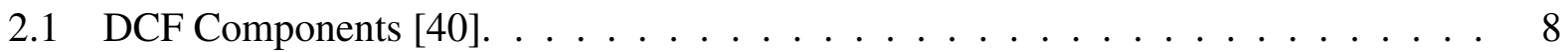

2.2 Basic Access Mechanism [14]. . . . . . . . . . . . . . . . . . . . 9

2.3 Example of Contention Window Growth. . . . . . . . . . . . . . . 10

2.4 Hidden Node Scenario. . . . . . . . . . . . . . . . . . . . . . . . 11

2.5 RTS CTS Scheme [14]. . . . . . . . . . . . . . . . . . . . . . . . 12

2.6 Block Diagram of IEEE 802.11 CSMA/CA within DCF [34] . . . . . . . . . . . 13

2.7 EDCA Mapping [15] . . . . . . . . . . . . . . . . . . . . . . 16

2.8 IFS Relationships [14]. . . . . . . . . . . . . . . . . . . . . . . . . 17

2.9 WAVE Architecture [35]. . . . . . . . . . . . . . . . . . . . 23

2.10 Channel Spectrum [31] . . . . . . . . . . . . . . . . . . . . . . . . . 24

2.11 CCH SCH Timing [33] . . . . . . . . . . . . . . . . . . . . . 25

2.12 Channel Cooordination [33] . . . . . . . . . . . . . . . . . . 27

2.13 Channel Priority for IEEE $802.11 \mathrm{p}[31] \ldots \ldots \ldots \ldots$

3.1 Block diagram of Simulator. . . . . . . . . . . . . . . . . . . 30

3.2 Top Level. . . . . . . . . . . . . . . . . . . . . . . . . . . . . . . . . . . 31

3.3 Measurement. . . . . . . . . . . . . . . . . . . . . 32

3.4 Road Side Unit. . . . . . . . . . . . . . . . . . . . . . . . . . . . . . . . 34

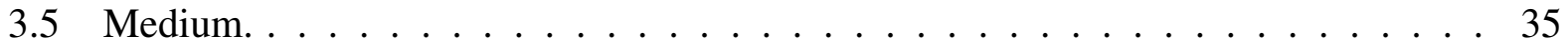

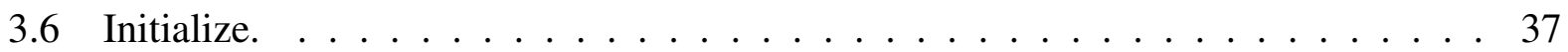

3.7 Source Poisson. . . . . . . . . . . . . . . . . . . . . . . . . . . . . . . 39

3.8 CSMA/CA within EDCA. . . . . . . . . . . . . . . . . . . 41

3.9 Device Main Page. . . . . . . . . . . . . . . . . . . . . . . 43

4.1 Average Access probability, Throughput, Average Collision Probability for different arrival rates and fixed number of devices. . . . . . . . . . . . . . . . 48

4.2 Average Waiting Time and Average Transmission Time for different arrival rates and fixed number of devices. . . . . . . . . . . . . . . . . . . . . . . 49

4.3 Average Access probability, Throughput, Average Collision Probability for different number of devices and fixed arrival rate . . . . . . . . . . . . . 50

4.4 Average Waiting Time and Average Transmission Time for different number of devices and fixed arrival rate. . . . . . . . . . . . . . . 51

4.5 Average Access probability, Throughput, Average Collision Probability different arrival rates and fixed number of devices equal to $7 \ldots \ldots$. . . . . . . . . 52

4.6 Average Waiting Time and Average Transmission Time for different arrival rates and fixed number of devices equal to 7 . 
4.7 Average Access probability, Throughput, Average Collision Probability for different arrival rates and fixed number of devices equal to 19. . . . . . . . . . . 54

4.8 Average Waiting Time and Average Transmission Time for different arrival rates and fixed number of devices equal to 19. . . . . . . . . . . . . . . 55

4.9 Performance Parameters for 802.11e EDCA. . . . . . . . . . . . . . . . . 56

4.10 Access Probability and Throughput for $802.11 \mathrm{p} \mathrm{CCH}$ for Duty Cycle equal to 0.6. . 58

4.11 Average Backoff Time and Average Backoff Phase for 802.11p CCH for Duty Cycle equal to $0.6 . \ldots \ldots \ldots$. . . . . . . . . . . . . . . . . . 59

4.12 AIFSN Restart Probability and No Collision Probability for $802.11 \mathrm{p} \mathrm{CCH}$ for Duty Cycle equal to $0.6 \ldots \ldots \ldots$. . . . . . . . . . . . . . . . . . 59

4.13 Access Probability and Throughput for $802.11 \mathrm{p} \mathrm{SCH}$ for Duty Cycle equal to 0.6. . 60

4.14 Average Backoff Time and Average Backoff Phase for 802.11p SCH for Duty Cy-

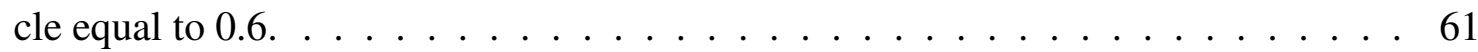

4.15 AIFSN Restart Probability and No Collision Probability for 802.11p SCH for Duty Cycle equal to $0.6 \ldots \ldots \ldots$. . . . . . . . . . . . . . . 61

4.16 Access Probability and Throughput for $802.11 \mathrm{p} \mathrm{CCH}$ for Duty Cycle equal to 0.5. . 62

4.17 Average Backoff Time and Average Backoff Phase for 802.11p CCH for Duty Cycle equal to 0.5 .

4.18 AIFSN Restart Probability and No Collision Probability for $802.11 \mathrm{p} \mathrm{CCH}$ for Duty Cycle equal to 0.5.

4.19 Access Probability and Throughput for $802.11 \mathrm{p} \mathrm{SCH}$ for Duty Cycle equal to 0.5. . 65

4.20 Average Backoff Time and Average Backoff Phase for 802.11p SCH for Duty Cycle equal to $0.5 \ldots \ldots \ldots \ldots \ldots \ldots$

4.21 AIFSN Restart Probability and No Collision Probability for 802.11p SCH for Duty

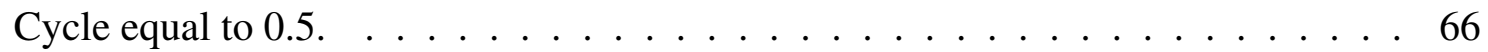

4.22 Access Probability and Throughput for $802.11 \mathrm{p} \mathrm{CCH}$ for Duty Cycle equal to 0.4. . 67

4.23 Average Backoff Time and Average Backoff Phase for 802.11p CCH for Duty Cycle equal to 0.4.

4.24 AIFSN Restart Probability and No Collision Probability for $802.11 \mathrm{p} \mathrm{CCH}$ for Duty Cycle equal to 0.4.

4.25 Access Probability and Throughput for $802.11 \mathrm{p} \mathrm{SCH}$ for Duty Cycle equal to 0.4. . 69

4.26 Average Backoff Time and Average Backoff Phase for 802.11p SCH for Duty Cycle equal to $0.4 \ldots \ldots \ldots \ldots \ldots \ldots \ldots \ldots$

4.27 AIFSN Restart Probability ann No Collision Probability for 802.11p SCH for Duty Cycle equal to $0.4 \ldots \ldots \ldots \ldots \ldots$. . . . . . . . . . . . . 70

4.28 Access Probability and Throughput for $802.11 \mathrm{p} \mathrm{CCH}$ for Duty Cycle equal to 0.3. . 71

4.29 Average Backoff Time and Average Backoff Phase for 802.11p CCH for Duty Cycle equal to $0.3 \ldots \ldots \ldots \ldots \ldots$. . . . . . . . . . . . . . . . . . . . .

4.30 AIFSN Restart Probability and No Collision Probability for $802.11 \mathrm{p} \mathrm{CCH}$ for Duty Cycle equal to $0.3 \ldots \ldots \ldots \ldots$. . . . . . . . . . . . . . 72

4.31 Access Probability and Throughput for $802.11 \mathrm{p} \mathrm{SCH}$ for Duty Cycle equal to 0.3. . 73 
4.32 Average Backoff Time and Average Backoff Phase for 802.11p SCH for Duty Cycle equal to $0.3 \ldots \ldots \ldots$. . . . . . . . . . . . . . . . . 73

4.33 AIFSN Restart Probability and No Collision Probability for 802.11p SCH for Duty

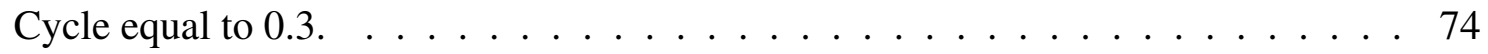

4.34 Access Probability and Throughput for $802.11 \mathrm{p} \mathrm{SCH}$ for Duty Cycle equal to 0.6. . 75

4.35 Average Backoff Time and Average Backoff Phase for 802.11p SCH for Duty Cycle equal to $0.6 \ldots \ldots \ldots \ldots \ldots \ldots$

4.36 AIFSN Restart Probability and No Collision Probability for $802.11 \mathrm{p}$ New Data Rate SCH for Duty Cycle equal to 0.6. . . . . . . . . . . . . . . . . . 76

4.37 Access Probability and Throughput for $802.11 \mathrm{p} \mathrm{SCH}$ for Duty Cycle equal to 0.5. . 78

4.38 Average Backoff Phase and Average Backoff Phase for 802.11p SCH for Duty Cycle equal to $0.5 \ldots \ldots \ldots$. . . . . . . . . . . . . . . . 78

4.39 AIFSN Restart Probability and No Collision Probability for $802.11 \mathrm{p}$ New Data Rate SCH for Duty Cycle equal to 0.5 . . . . . . . . . . . . . . . . . . 79

4.40 Access Probability and Throughput for $802.11 \mathrm{p} \mathrm{SCH}$ for Duty Cycle equal to 0.4. . 80

4.41 Average Backoff Time and Average Backoff Phase for 802.11p SCH for Duty Cycle equal to $0.4 \ldots \ldots \ldots \ldots \ldots$. . . . . . . . . . . . . 80

4.42 AIFSN Restart Probability and No Collision Probability for $802.11 \mathrm{p}$ New Data Rate SCH for Duty Cycle equal to $0.4 \ldots \ldots$. . . . . . . . . . . . . . . 81

4.43 Access Probability and Throughput for $802.11 \mathrm{p} \mathrm{SCH}$ for Duty Cycle equal to 0.3. . 82

4.44 Average Backoff Time and Average Backoff Phase for 802.11p SCH for Duty Cycle equal to $0.3 \ldots \ldots \ldots \ldots \ldots$. . . . . . . . . . . . . . . 82

4.45 AIFSN Restart Probability and No Collision Probability for 802.11p SCH for Duty Cycle equal to $0.3 \ldots \ldots \ldots \ldots \ldots \ldots \ldots$ 


\section{Chapter 1}

\section{Introduction}

According to the recent statistics of Global Issues [4] in May 2011, each day 3500 people are killed and 13700 injured in road accidents around the world. With the increase in the population, the number of vehicles over the road are bound to increase, thereby increasing the probability of more crashes and fatalities. With this main factor along with other factors, extensive research was started in the field of VANETs (Vehicular Adhoc Networks).

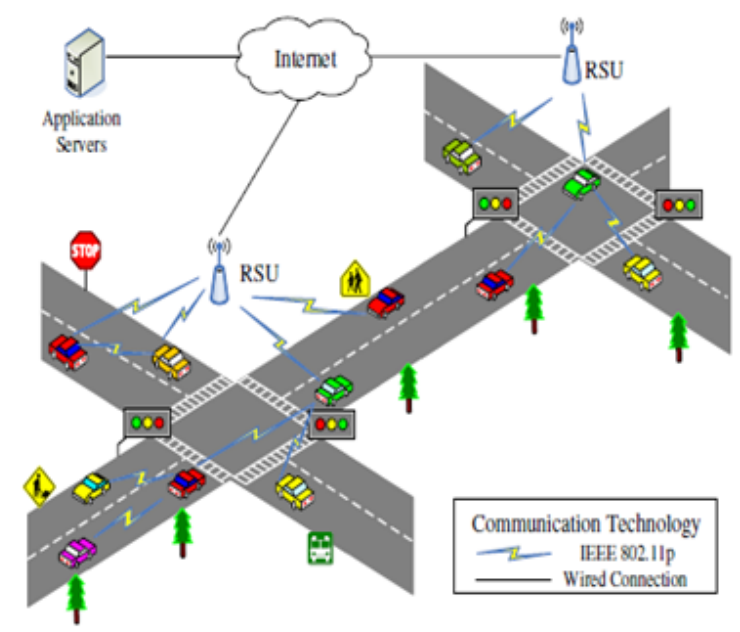

Figure 1.1: VANETs Scenario.

It's still in the early phases of development as it's a very wide field. Moreover, along with providing the safety applications, there was a call for providing multimedia services while driving so 
as to make driving a safe and pleasant experience. Basically, there are two types of communication possible in VANETs, vehicle to infrastructure (V2I) and vehicle to vehicle (V2V). Each vehicle has a On Board Unit (OBU), which helps in communication with other OBUs and with Road Side Units (RSU) installed at road side and sometimes at a busy intersection to help in the communication. Once the communication starts, the OBU to OBU communication can also be used to send messages where the installation of the RSU is not possible due to topographic features or cost issues. However in this work, we deal with the OBU to RSU communication only, with traffic flowing uplink and downlink.

\subsection{VANET Characteristics}

MANETs (Mobile Adhoc Networks) can be used where quick deployment is required. Its importance can be realized when it is deployed during wars or any other natural disaster which otherwise is not possible. VANETs are a subclass of MANETs. They both have a lot of common characteristics. Here we discuss some of the important characteristics of VANETs.

- Mobility: As vehicles keep on moving from one place to another, their topology keeps on changing and it never remains same for a long duration of time. As a result of this, it is very important to create efficient and fast connections with the RSU and other OBU for communication. Moreover issue of handoff becomes very critical when mobility is taken into consideration as the vehicles would continue to move in and out of different RSU's coverage area.

- Latency: As critical messages needs to be send on the $\mathrm{CCH}$, it is the most important characteristic of the VANETS to have low latency otherwise all its applications would be useless. So, if an emergency message of an accident ahead takes more time than the vehichle to reach that place the utility of that message is zero as the driver would have no prior knowledge of 
this scenario and this could lead into a more fatal accident.

- Security: Like all the wireless networks, there is a possibility of attacks on VANETs. As communication has to take place between OBU and OBU and OBU and RSU, any adversary might try to use the data to its advantage. As communication can be V2I or V2V, there is a possibility of attack by adversary who might use the data transmitted on $\mathrm{SCH}$ for taking some valuable private information or on $\mathrm{CCH}$ where it might send some bogus warnings and messages so as to advantage of this situation for his own good.

- Infrastructureless: If the RSU is not installed in a particular area because of cost or any other topology issues, even then the communication can take place as OBU can transmit messages to the other OBU. Due to cost issues, RSU installation is very expensive, so a partial deployment of RSU can be done at busy places and the further communication can be done in V2V mode. Thus, VANETs can survive without a fixed static infrastructure with ease.

- No Power Constraints: Unlike wireless sensor networks there are no power constraint in VANETs as vehicle can draw power from vehicle's battery which in most cases is a reliable source of power.

- Density: Due to the mobility factor in VANETs, the density of the nodes is variable as it keeps on changing. Hence, the density of vehicles is not the same at the same place at a different instant of time.

\subsection{Applications}

The main motivation for research in VANETs is not only the safety aspect of driving, but also various other multimedia applications that can make driving a lot more enjoyable and easier. Due 
to large number of applications, VANETs have attracted a lot of attention from researchers to work in this field.

- Active Security: It is one of the most important application of VANETs as it has a direct consequence on the road safety. The main aim is to warn the vehicles of a danger so that they can take appropriate action or at least reduce the impact of the danger. Steep curves, low bridge warning or road conditions can be termed as low priority dangers as compared to the situation when vehicles transmit the information about an accident which has the highest priority as it may impact the approaching vehicles safety. When this warning is send in advance to the other vehicles, they can take appropriate actions to avoid these type of dangers and ensure the safety of vehicle and its occupants.

- Public Safety and assistance: Emergency vehicles like police car or fire brigade need to reach the destination as soon as possible. Although these vehicles have a siren to inform other vehicles to give free path to it but message passing in advance between vehicles would help the emergency vehicles as other vehicles would clear the lane for it in advance. Also, VANETs provide a very important advantage as it can be used at a toll collection point. When a vehicle passes through a special RSU it scans the Electronic License Plate of the vehicle and gives a automated receipt for the toll money at the counter which helps in reducing the delays at the counters and helps in avoiding big line ups.

- Improved Driving: Information about lane changing and merging traffic can be useful for other vehicles which would eventually help out in easing the traffic on the roads as the approaching vehicles would already know the traffic conditions of an area and if congested would take alternative routes to reach his destination. However, security and privacy issues have to be taken into consideration as an adversary may try to take advantage of this condition for his own benefit.

- Internet Access: Internet access is provided in VANETs. Although right now few issues are 
being resolved so it has not been fully implemented but in the near future this would be one of the most important application of VANETs. The approach that is being used establishes a connection of OBU with RSU which would be connected to the internet and provide access to OBU with sufficient security and privacy.

- Roadside Services Finder: This application can be used to find hospitals, gas stations and restaurants etc. Roadside service database would be installed in the area which would interact with the respective RSU. When the request comes for service request, the query is searched from the database and the result is sent to RSU which propagates the result to the OBU. Service providers may come into collaboration with the RSU and with the help of RSU send advertisement provided by them in the nearby areas. This type of application would help in generating revenue and carry this industry forward.

\subsection{Problem Statement}

The problem that we have addressed in this thesis is that we want to find the best duty cycle on which a device should work with control and service channels with time multiplexing between them so as to obtain the optimum performance of the network. As the device would alternately switch between these channels, no critcal messages should be lost as they as life saving messages and we continue to receive multimedia messages with any disruption in service.

\subsection{Solution Approach}

In order to obtain the solution for the above mentioned problem, first we started with 802.11 DCF. The reason we started with DCF is that the CSMA process that would be used in $802.11 \mathrm{p}$ is similar to the 802.11 DCF. The only difference is that DIFS in DCF is replaced by AIFS in 802.11p and it has different $\mathrm{CW}$ for each $\mathrm{AC}$. We further analysed the saturation condition to observe the 
performance of the network in that condition. The limitations of DCF, mainly due to lack of QoS motivated us to develop 802.11e EDCA. The reason we chose 802.11e EDCA was that it has four $\mathrm{AC}$ along with same AIFS and CW parameters which are also required in $802.11 \mathrm{p}$. Due to lower QoS and lack of multichannel capability we decided to finally modify this to $802.11 \mathrm{p}$. Once we had multichannel capability we could easily perform the channel switching to evaluate the performance on control and service channels with different duty cycles.

\subsection{Thesis Contribution}

To solve the problem mentioned, we developed a simulator as per the IEEE $802.11 \mathrm{p}$ standard that provides support to critical and multimedia messages and applications. We achieve this varying the duty cycle so that the devices are able to adapt to the dynamic environment of VANETs. By varying the duty cycle we can achieve better quality of service and optimized data. Our work builds on the step by step approach as mentioned in the previous section so as to find out the best duty cycle on which the control and service channels should work.

\subsection{Thesis Organization}

In Chapter 2, all the significant related work has been mentioned. In Chapter 3, we discuss the design of the simulator which we developed for measuring the parameters of the network followed by Chapter 4 in which we discuss the results that we obtained and Chapter 5 has the conclusion that we can derive from this work. 


\section{Chapter 2}

\section{Background and Related Work}

\subsection{Distributed Coordination Function}

The IEEE 802.11 is a standard proposed for wireless local area network. Due to low cost and easy deployment it gained a lot of popularity. IEEE 802.11 Medium Access Control (MAC) uses two medium access methods, the Distributed Coordination Function (DCF) and the optional Point Coordination Function (PCF). DCF is an asynchronous data transmission function which suits delay insensitive data, whereas the Point Coordination Function (PCF) is used in time-bounded applications. DCF defines two mechanisms for packet transmission, the basic access and the Request-ToSend/Clear-To-Send (RTS/CTS) reservation scheme.

There have been various attempts to model 802.11 DCF either through simulation or analytical models. The authors in [11] used bursty data to observe the throughput, assuming that there is no hidden node problem, and their emphasis was more on the physical layer rather than the MAC layer. In [12], the performance evaluation was done, but the results obtained showed that their model performed poorly with the small sized packets. The most notable work was done in [8] in which the author modelled the DCF with the assumption of unlimited retry limit i.e the device would keep on making unlimited number of attempts to send the packet until it is successfully transmitted. This work was extended by [9] and it included the retry limit as per the standard [14]. The work of [10] takes into account the exponential backoff details and provided the saturation throughput analysis. So taking all this work into account, we developed our own model to measure the performance parameters of the network and further extended it as per our requirements. The 
components of the architecture of DCF are very important. The various parts of its architecture are explained below.

\subsubsection{DCF Components}

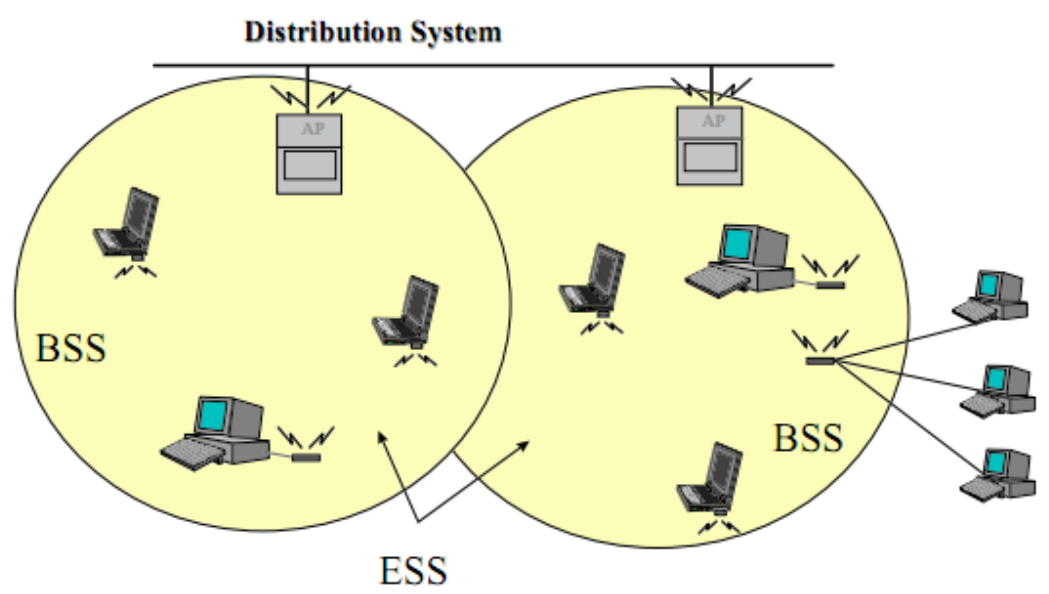

Figure 2.1: DCF Components [40].

- STATION/DEVICE: The most important part of the network is the station. It is responsible for communication as it sends the data through medium. The network may have many stations which work in each other's vicinity so as to access the medium to transmit data.

- BASIC SERVICE SET: This constitutes the stations that communicate with each other. If there is no wired connection and all the stations are mobile the BSS is termed as independent BSS (IBSS) which is an adhoc network created for some specific purpose. If the BSS consists of an access point (AP) then it is known as infrastructure BSS.

- Access Point (AP): The main purpose of access point in the BSS is for communication. If a station has to communicate with another station, the station needs to send the data to the access point which in turn sends it to the specific device. It may be argued that it might be better to perform this transmission without the access point but the main advantage of 
access point storing the data is far more than its disadvantage of using more bandwidth for communication.

- Extended Services Set (ESS): The different BSSs together form the ESS. It main function is to enable the communication of AP in different BSSs with the help of distribution system (DS) so as to enable the mobile stations to move from one BSS to another.

- DISTRIBUTION SYSTEM: It is responsible for communication between APs in different BSS.

\subsubsection{Basic Access Mechanism}

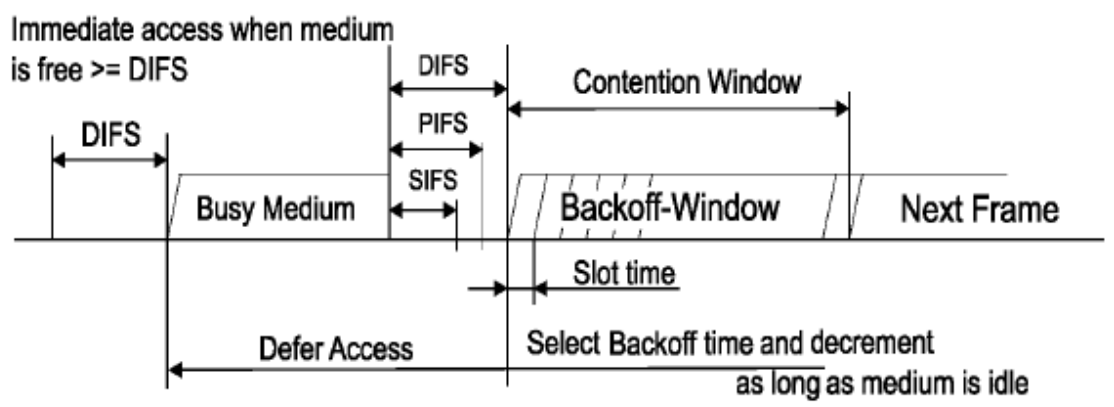

Figure 2.2: Basic Access Mechanism [14].

As shown above in this type of communication just after the successful transmission when ACK is received the device waits for the medium to be idle for DIFS period of time. If this happens, the device transmits the packet. However, if the medium is busy, the device waits for the current transmission to end and then to be idle for DIFS and then performs a backoff process so as to randomize the transmission to avoid the collision of packets while transmitting. The random backoff number is chosen such that it is in the range of $(0, \mathrm{CW}-1)$ according to uniform distribution. 


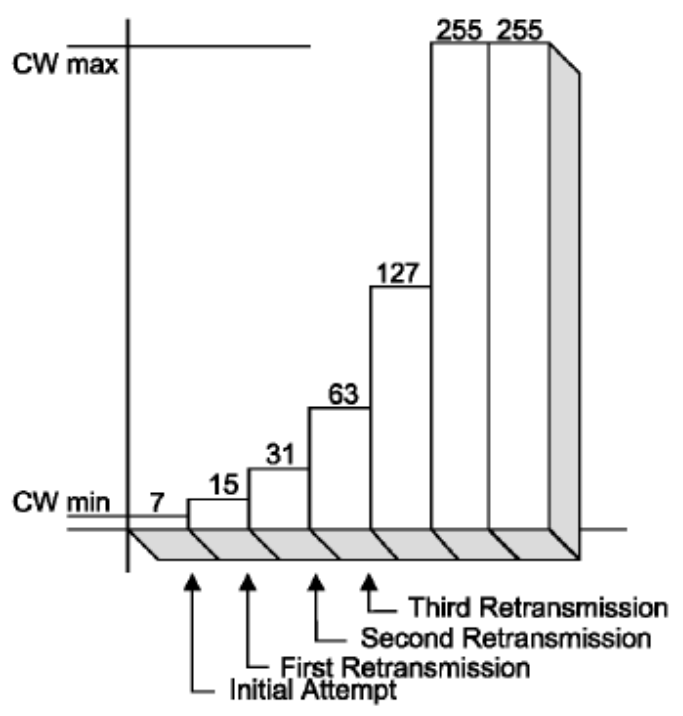

Figure 2.3: Example of Contention Window Growth.

The CW parameter takes the initial value of aCWmin. The CW shall take the next value in the series every time an unsuccessful attempt to transmit the packet is made i.e collision of packets take place, causing either the retry counter to increment or until the $\mathrm{CW}$ reaches the value aCWmax. The maximum retry limit is the number of attempts made to transmit the packet after which the packet is dropped. Once the $\mathrm{CW}$ reaches the CWmax value, it maintains the same value until it is reset. This is very important factor under high load conditions. Once the random backoff count becomes zero the device transmits the packet. However, if during the backoff count the medium becomes busy the random backoff counter is frozen and when the medium becomes IDLE for DIFS, then the backoff counter is decremented again till it becomes 0 and after that the packet is transmitted. In response to this data packet, an ACK is sent after SIFS period of time confirming the successful transmission of the packet. The main advantage of using this technique is when the packet size is small because using RTS/CTS scheme would add overhead even for the short data 
packets resulting in wastage of network bandwidth. However, this mechanism has a flaw known as hidden node problem [5] which is described in the next section.

\subsubsection{Hidden Node Problem}

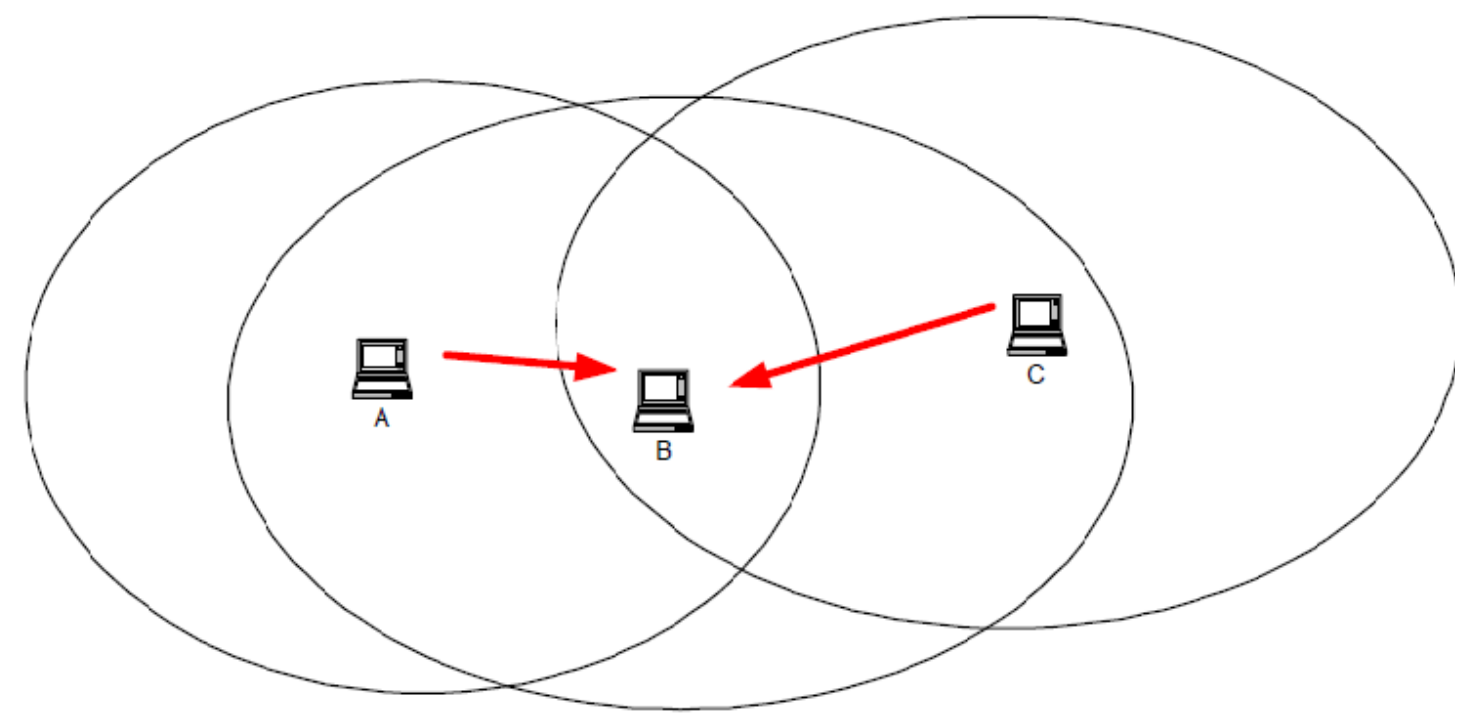

Figure 2.4: Hidden Node Scenario.

In this type of scenario, the problem that arises is that stations $\mathrm{A}$ and $\mathrm{C}$ are not able to hear each others transmission, which increases the chance of collision. This is known as hidden terminal problem [5]. The authors in [6] performed experiments to arrive at the conclusion that when the number of hidden terminals is large or the offered load is high, the RTS/CTS access method provides better performance in comparison to the basic access method. Similarly the analysis in [7] shows that the RTS/CTS scheme effectively improves the hidden node problem. As a solution, the use of management frames like Request-to-Send and Clear-to-Send was started before transmitting the data packets. When a RTS is sent and destination sends the CTS in response, all the other stations defer their transmission till the ACK is received. If RTS collides, the backoff procedure is repeated. 


\subsubsection{RTS/CTS Scheme}

In this scheme, the medium is reserved for a particular device which succeed in sending the RTS and receive CTS in return. Once the CTS comes back, the data packet is send to the destination which sends an ACK to the source to acknowledge that the packet was successfully transmitted.

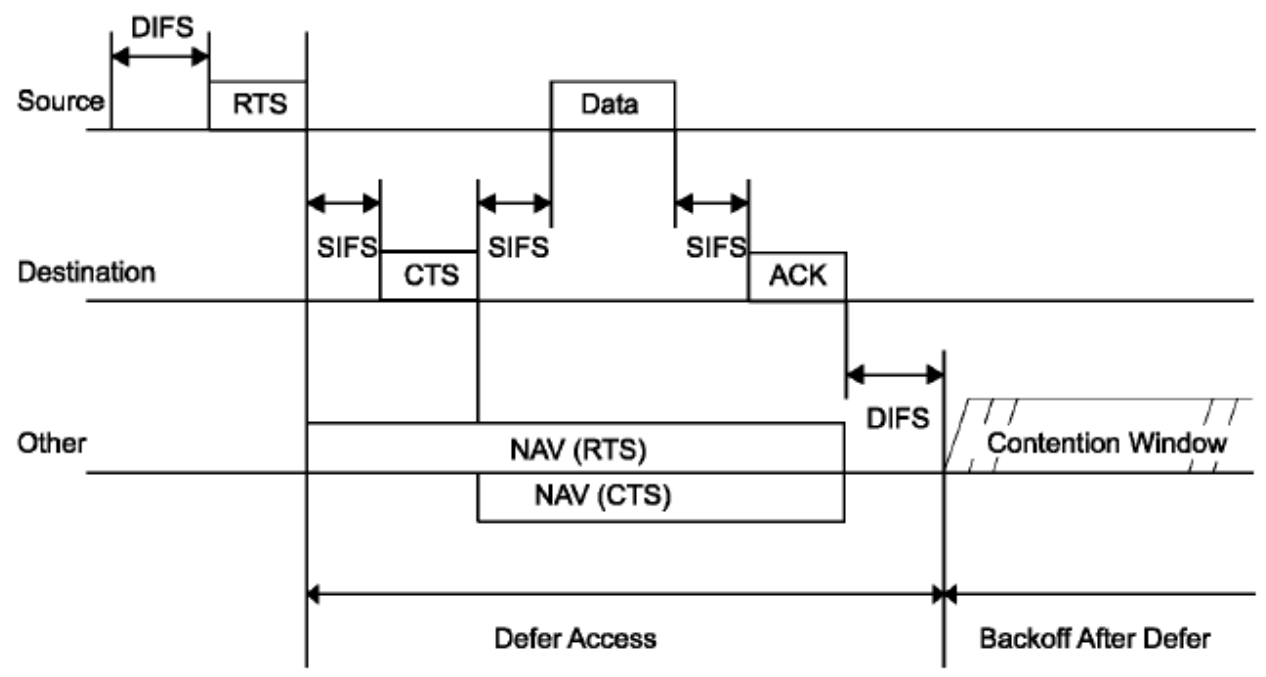

Figure 2.5: RTS CTS Scheme [14].

The main advantage of this technique is that when the packet size is large for transmission then prior to its transmission RTS is sent and collision occurs only on the RTS which is small in size rather than the data packets. 


\subsubsection{CSMA/CA for DCF}

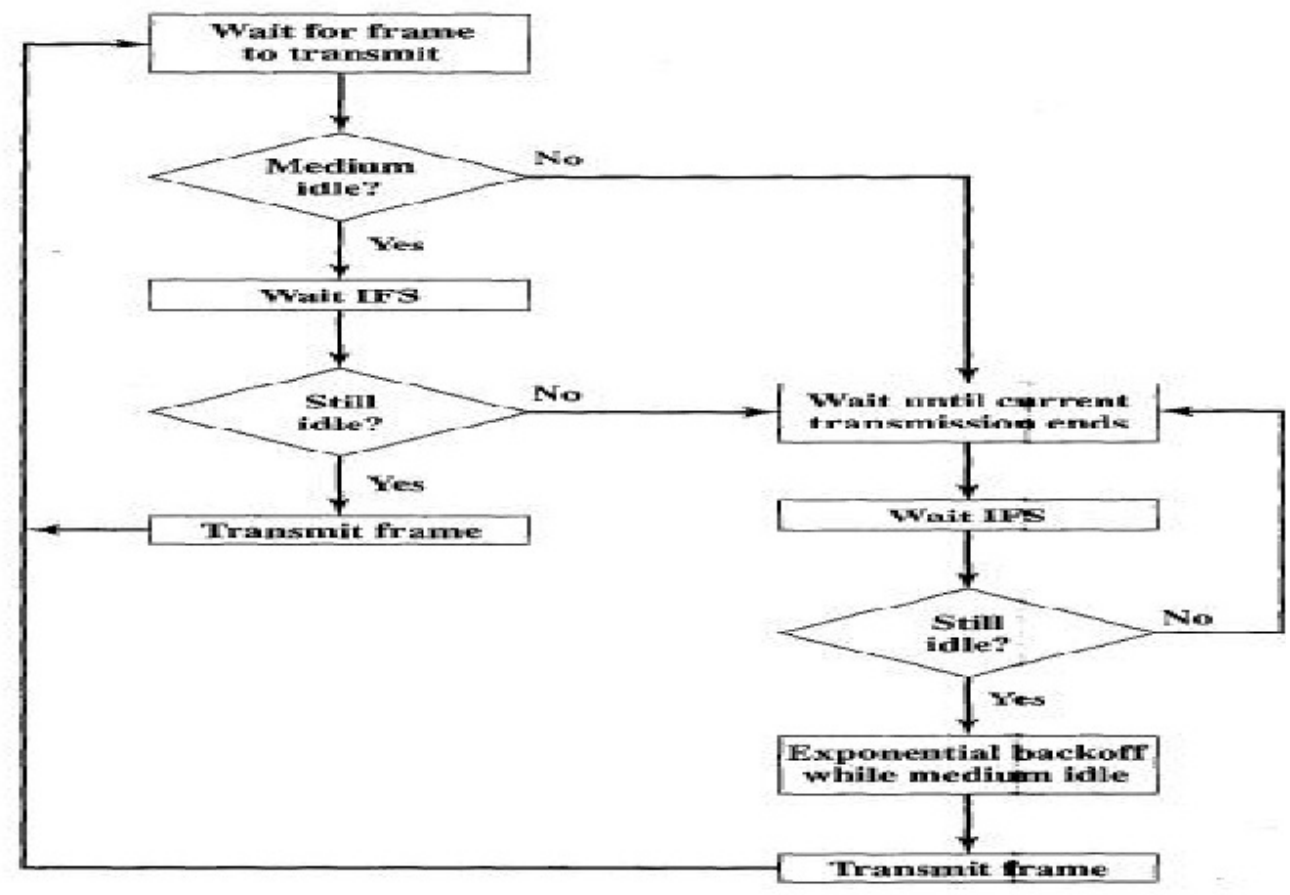

Figure 2.6: Block Diagram of IEEE 802.11 CSMA/CA within DCF [34].

The device uses the CSMA/CA process to capture the medium. If the medium is idle for DIFS and it is idle even after that then the device transmits immediately. However, if the medium is not idle, the device waits for current transmission to end. The device keeps sensing the medium and when it becomes idle for DIFS, it starts its backoff process. The device transmits when the random count becomes 0 . If the random count is interrupted, the countdown is frozen and the same procedure is repeated such that the random countdown resumes from where it was frozen. If the packet collides, then in the next phase the $\mathrm{CW}$ is doubled and this keeps on going until the maximum $\mathrm{CW}$ is reached or the maximum retry limit is achieved. 


\subsubsection{Limitations of DCF}

The 802.11 DCF does not support QoS which is very important for VANETs. This is the primary reason why 802.11 cannot be used in VANETs as we require different types of data for various applications in VANETs which is not possible if we use 802.11 DCF in VANETs. The data that is transmitted has to go through the access point to reach the destination. However the access point has no special priority and it has the same probability of transmission as any other station in the network. This would be problematic in a busy and congested network and so it is not feasible for VANETs.

\subsubsection{Point Coordinated Function}

The optional PCF can support time sensitive applications to a certain extent but it had some flaws. The PCF is split into contention free period (CFP) and contention period (CP). The stations which can transmit during CFP should be polled by point coordinator (PC). PCF tries to provide some sort of priority but it clearly is not the best option to QoS. Due to the limitations of PCF described in [13], a new standard, 802.11e was developed for providing QoS.

\subsubsection{Limitations of PCF}

Superframe length has to be adjusted as it affects the delay experienced by the applications using PCF. The polling mechanism used by PCF is round robin which may not always be feasible depending on the requirements. The transmission in the CP interval affects the time when CFP starts causing delay for the stations to be polled during CP hence introducing unpredictable delay in CFP. The alternating CFP and CP can introduce a lot of overheads if the super frame size becomes small and as we know to provide short delay superframes size should be small in PCF. 


\subsection{EDCA}

Looking at the bigger picture of developing 802.11p simulator we took the approach of developing 802.11 DCF and then modifying it to 802.11e and extending it to 802.11p. According to the 802.11e channel structure, the time is divided into Contention Free Period and Contention Period (CP). The EDCA protocol works in the contention period. In the following sections, we will be discussing about EDCA. The requirements of enhancements to 802.11 for QoS purposes led to the development of 802.11e standard [15]. IEEE 802.11e supports service differentiation by assigning data different priorities depending upon the requirements. Four different Access Categories (AC) have been defined for data traffic, having different priorities which are independent of each other. Access to the medium is then granted based on the priorities of data traffic, such that each frame with a particular priority is mapped to an access category and a different set of contention parameters is used by each AC to contend for the medium.

ACCESS CATEGORIES: EDCA has four different type of access categories, namely voice (VO), video (VO), best effort (BE) and background (BK). The data is mapped to different AC category depending on the data type. The priority as mentioned in the standard is shown in the Table 2.1

The voice access category has the highest priority followed video, best effort and background access category. The priority assigned to these categories depends on various EDCA parameters like AIFS, CWmin and CWmax and TXOP. Each device has four AC which are contending for the medium as per the csma/ca process of 802.11 DCF with EDCA parameters replacing the parameters used in DCF. 


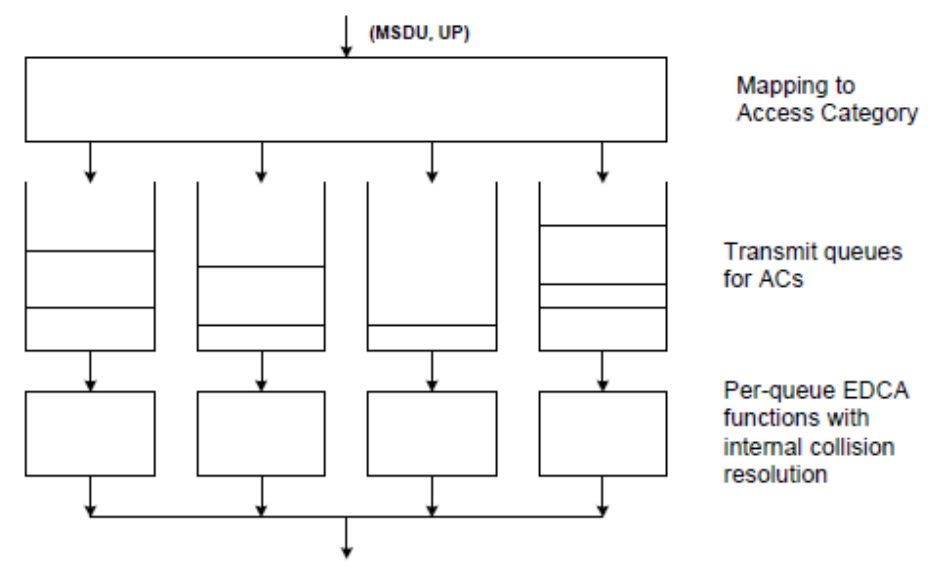

Figure 2.7: EDCA Mapping [15].

Table 2.1: Access Category Used In Our Model.

\begin{tabular}{cccc}
\hline \hline Priority & User Priority & Access Category & Designation \\
\hline Lowest & 1 & AC_BK & Background \\
$\cdot$ & 0 & AC_BE & Best Effort \\
$\cdot$ & 2 & AC_VI & Video \\
Highest & 3 & AC_VO & Voice
\end{tabular}

\subsubsection{Interframe Space Relationship}

Before understanding the priority access in $802.11 \mathrm{p}$ we need to see the relationships between various IFS as described in [14]. The time interval between frames is known is Interframe Space (IFS). The device senses the medium idle by carrier sensing mechanism which is used for a specific interval of time. Here, different IFS are discussed which play a very important role in providing priority to different wireless media. Before, we understand the role of IFS in $802.11 \mathrm{p}$ for priority 
access, we will discuss all the other IFS which are available for wireless media.

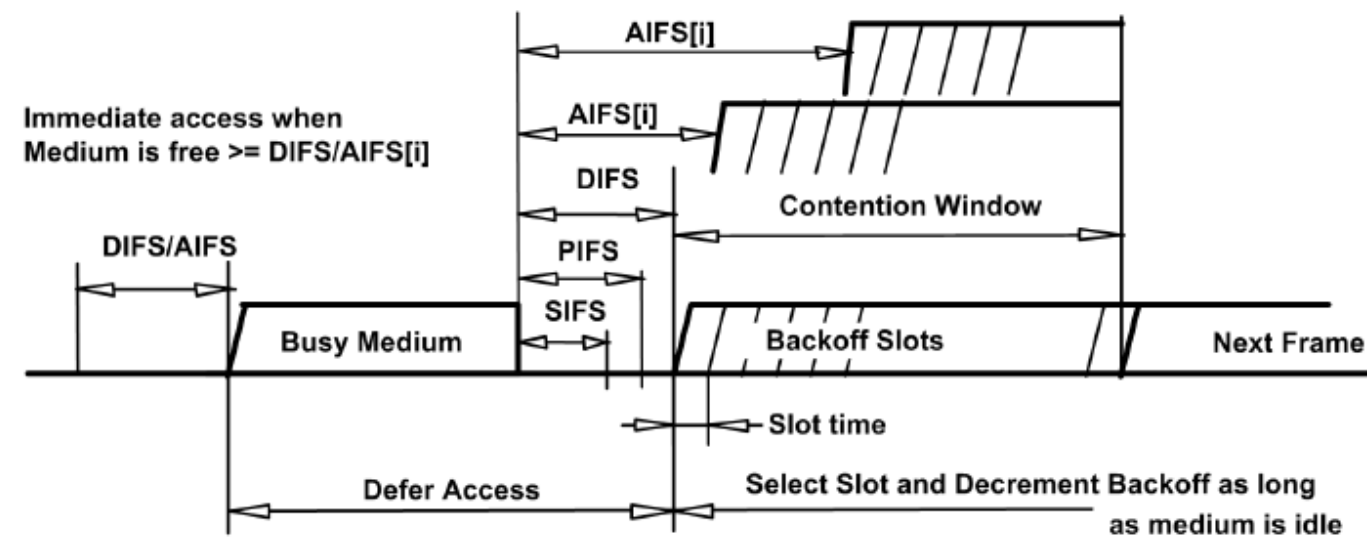

Figure 2.8: IFS Relationships [14].

- SIFS: It is known as Short Interframe Space. This is the shortest of all the IFS. It can be defined as the time from the last slot of the packet transmitted to the beginning of the first slot of the following packet. It plays a very important role as SIFS does not allow the other devices to access the medium when a current transmission is going on. In this way, priority is given to the current frame transmission and does not allow the new transmission to start till the current transmission gets completed.

- PIFS: It is known as Point (Coordinated Function) Interframe Space. The PIFS is used by devices which are operating in PCF, to access the medium at the start of the CFP or for channel switch announcement frame.

- DIFS: It is known as Distributed Interframe Space. This is used by the devices operating in DCF for starting a new transmission. A device has to sense the medium idle for DIFS after 
a previous packet transmission has been completed successfully.

- AIFS: It is known as Arbitration Interframe Space. It is used by devices for QoS to transmit data. The device obtains the TXOP for AC if the medium is sensed idle for AIFS after the previous transmission is successful.

- EIFS: A device in DCF would use EIFS when a previously transmitted frame is corrupted. The device would not start the transmission till the NAV value used in the transmission expires followed by EIFS. This provides the device enough time to resynchronize itself to the actual state of the medium and after that the normal DIFS or AIFS countdown is done for the received frame.

\subsubsection{EDCA Parameters}

- AIFS (Arbitration Interframe Space): This parameter is similar to what we had as DIFS in DCF. This is the minimum time for which a device has to detect medium idle before it can start its transmission or its backoff. The difference from DCF is that DIFS value is fixed whereas AIFS value is different for different ACs, proving to be an important factor in providing priority to ACs. This AIFS value plays a very crucial role as the higher priority ACs have shorter AIFSN value and hence they have to wait for less time to start the transmission or backoff procedure and would have less delay as compared to lower priority ACs.

- CW (Contention Window): The CWmin and CWmax are fixed in the DCF but in this standard different ACs have different values for them. The higher priority ACs have smaller contention window as compared to the lower priority ACs. The lower CW would cause the higher priority AC to choose a smaller random number for backoff which enables the highest priority AC to wait for the shortest period of time when the medium becomes idle as it has the shortest AIFSN value. In the case of collision, the $\mathrm{CW}$ for higher $\mathrm{ACs}$ is selected in such 
a way that its CWmax is less than the CWmin of the lower priority ACs. In this way even after the higher priority $\mathrm{ACs}$ reaches its maximum $\mathrm{CW}$ value it still has the higher priority over the lower category AC. However, in the case of lower category suffering from repeated collision, its performance degrades as it has to select a random backoff number for a higher range as $\mathrm{CW}$ doubles after every retransmission attempt leading to starvation. The process of selecting the pseudo random integer from a uniform distribution over the interval $[0, \mathrm{CW}]$ is done with the following formula.

Backoff Time $=$ Random ()$\cdot \operatorname{aSlotTime~}(2.1)$

where $\mathrm{CW}$ is an integer within the range of aCWmin $\leq \mathrm{CW} \leq \mathrm{aCWmax}$ aSlotTime is the slot time

- TXOP (Transmission Opportunity): It is the duration for which a device would transmit after successfullly winning the medium. As per the standard the lower priority ACs have this value set as 0 whereas higher priority ACs have non zero value, providing them with the opportunity to transmit many frames at a time after winning the medium. However, in our model for 802.11e, we assume that TXOP as 0 for every AC so that a device would send only one packet at a time. When we extend this model to $802.11 \mathrm{p}$, we continue with the same value as in $802.11 \mathrm{p}$, TXOP is defined as 0 .

There is one more factor in the design of this model. It may happen that one or more ACs may finish the backoff process at the same time and try to transmit at the same instant. In this scenario, 
Table 2.2: Default Values of EDCA Parameters.

\begin{tabular}{cccc}
\hline \hline ACCESS CATEGORY & CWmin & CWmax & AIFSN \\
\hline Voice(VO) & 3 & 7 & 2 \\
Video(VI) & 3 & 7 & 3 \\
Best Effort(BE) & 7 & 15 & 6 \\
Background(BK) & 15 & 511 & 9
\end{tabular}

as per the standard, the higher priority $\mathrm{AC}$ sends the packet and the lower priority AC behaves as if it suffered a collision and follows the backoff procedure by doubling its CW. This is known as virtual collision. In our model for $802.11 \mathrm{e}$, we have not taken into account this virtual collision due to implementation issues and we assume that there are no virtual collision in our model and we deal with only external collisions.

The fact is that the performance of the 802.11e network is best in non saturated conditions [16][17]. The work in [18] was an extension of the Bianchi model [8] with different CWs for every AC. The authors of [19] implements the Bianchi model [8] with certain limitations. For instance, it does not consider the backoff freezing alongwith TXOP. The work in [20] tries to model it but it does not take into account the AIFS procedure as stated in the standard. In their model, when the medium becomes busy during AIFS countdown, the counter freezes and then again start decrementing from the same value whereas it should restart from the initial value. In [21], the author considers equal AIFSN values for all ACs. Also in [22], the authors omit many system details and does not invoke the TXOP differentiation, assuming a single AC active in a station. They analyzed only 2 ACs in the network. Virtual collisions and retry limit are also neglected in [23] and they try to introduce TXOP in the model but due to complexity of the model authors could not trace its affect on the model. There are many other models derived from these models, but these are the most significant models which we have discussed. 


\subsection{Description of IEEE 802.11p standard}

This is a new standard and the final $802.11 \mathrm{p}$ amendment was released on 15 July 2010, which is an approved amendment of 802.11 standard defining changes to support Intelligent Transportation Systems involving data exchange between vehicles and road side units. The packet transmission as explained in [24] follows the broadcast transmission which means that there would be no RTS/CTS and whether packet transmission is successful or not would remain unknown causing more collisions. In [24], they discussed to prevent channel saturation and introduced priority for emergency messages. Also in [25] [26], the performance evaluation for 802.11p broadcasts is done showing the effect of node population on the network.

In [27], the SCH are assigned on a reservation based TDMA scheme. The main drawback of this scheme is that it is done at the cost of $\mathrm{CCH}$ as it is fractioned which increases the chances of the safety messages not being transmitted and increases the latency. This could lead to starvation of emergency messages which are bandwidth sensitive while waiting for the $\mathrm{SCH}$ to complete its transmission. The channel access discussed in [28][29] were designed in such a way that a device would stay on the $\mathrm{SCH}$ as long as it does not complete its transmission before switching to $\mathrm{CCH}$. The addition that was made in [29] was that RSU can monitor both $\mathrm{CCH}$ and $\mathrm{SCH}$ simultaneously and broadcast the emergency messages when required which was not done in [28]. In [30], during the $\mathrm{CCH}$ interval on-board units notify the roadside unit about their interest in the advertised services that can be used, which increases the load on the control channel and it affects the transmission of the safety messages. There are many other schemes for switching between the $\mathrm{CCH}$ and $\mathrm{SCH}$, but we have discussed the most important ones here. In our approach we would be using different duty cycles for switching between $\mathrm{CCH}$ and $\mathrm{SCH}$ so as to analyse which is the best duty cycle on which $802.11 \mathrm{p}$ should work so as to have the optimum performance. In [31], the duty cycle was used to switch between channels using an analytical model and in this work we have extended that approach to measure the network parameters using our simulation model. In 
[32], the authors mention the problem of using broadcasting.

- If broadcasting protocol is being used then retransmission of packets is not possible as no management frames are used in this protocol. Even if this protocol is enhanced in such a way that on the successful reception of packet $\mathrm{ACK}$ is sent then the problem of receiving too many ACK would arise as all the devices would send back the ACK when they receive the packet causing collisions of ACKs.

- The hidden node problem that we discussed for the DCF would arise and it can be resolved only if we use RTS/CTS scheme and not broadcast as no channel reservation would be possible in broadcasting before sending the packet.

- The CW size would always remain the same as there is no retransmission and there is no mechanism to detect the failed transmission. The transmission that would take place would always be with CWmin.

\subsection{Operation of IEEE 802.11p}

For applications looking to create a connection, they should announce it on the $\mathrm{CCH}$ in a WBSS advertisement frame. The frame has all the details for connection setup. A device can be a part of only one WBSS at a time, but a WBSS can have many devices. WBSS are of two types, persistent and non persistent. The main advantage of persistent WBSS is that it is announced periodically in every $\mathrm{CCH}$ interval whereas non persistent WBSS is announced only once when it is initiated. 


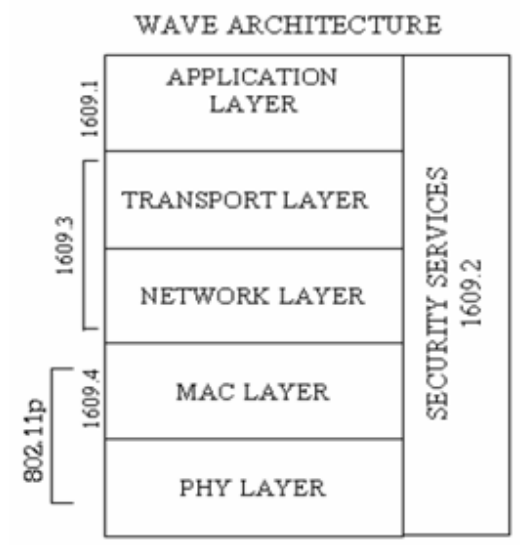

Figure 2.9: WAVE Architecture [35].

Upper layer protocols and services requirements of WAVE are described by IEEE 1609 standards. The IEEE 802.11p provides foundation for WAVE signaling technique and interface functions that are controlled by the IEEE 802.11 MAC. The WAVE architecture uses standard IEEE 802.11p and 1609. The 1609.1 [36] define the message data format and define the services and interfaces of the resource manager. Protocol 1609.2 [37] defines secure message formats and message exchange. Protocol 1609.3 [38] defines the routing and transport services. When fully functional it can be an alternative to IPV6 packets. Protocol 1609.4 [39] enables the operation of upper layers across multiple channels. CSMA/CA is used by 802.11p as a medium access scheme for link sharing and uses control and service channels to achieve that. 


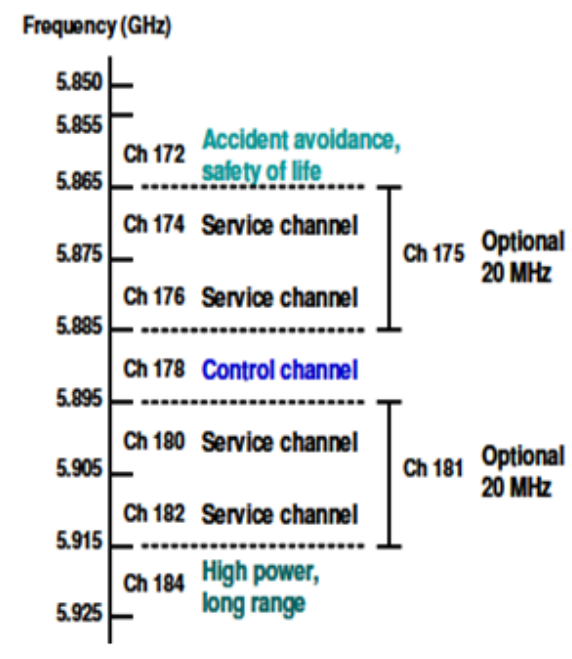

Figure 2.10: Channel Spectrum [31].

As vehicular safety applications cannot establish long connections and efficient connection, the setup is difficult to establish the Wireless Access in Vehicular Networks(WAVE) consisting of 802.11p MAC protocol was proposed. The WAVE operates in 5.850-5.925 GHz which is divided into one Control Channel $(\mathrm{CCH})$ and six Service Channels $(\mathrm{SCH})$. Ch 178 is the $\mathrm{CCH}$ channel whereas all the other six channels are the SCH. Ch 172 and $\mathrm{Ch} 184$ are reserved for future use. The $\mathrm{CCH}$ is used for transmission of safety and emergency messages whereas $\mathrm{SCH}$ is used for sending infotainment data which is not application specific. Each node has four traffic classes namely Voice (VO), Video (VI), Best Effort (BE), Background (BK). In our work we would evaluate multi channel devices. When the device would work as shown in sync interval, it would be switching between the control and service channel. 


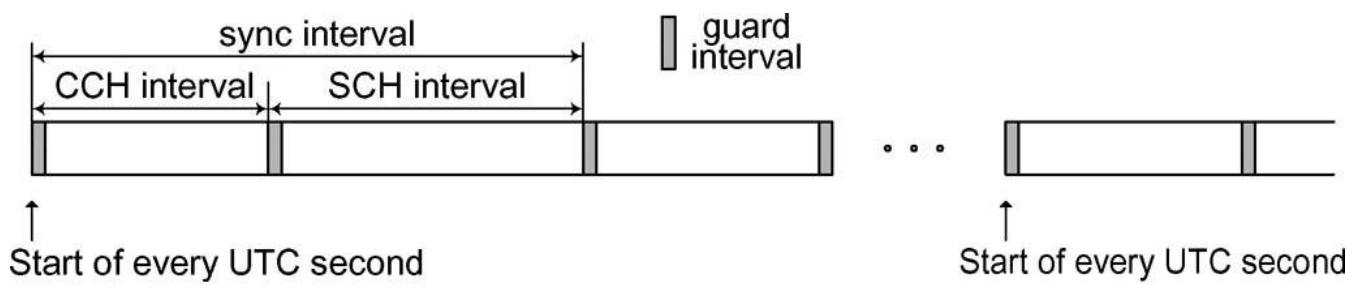

Figure 2.11: CCH SCH Timing [33].

The channel coordination that is explained in the following part is dependent on the channel synchronization. The Fig 3.3 shows the timing relationship between $\mathrm{CCH}$ and $\mathrm{SCH}$. As the devices have to synchronize to a common time UTC (Coordinated Universal Time) is used as a common reference time. At the very beginning of the interval, we have a guard period which takes into account all the inaccuracies and variations at the beginning. The other important function of the guard period is that if any transmission is not completed in the given interval then it can be used to complete the transmission if possible. Also, no new transmission can take place in this period and this helps in randomizing the backoff process of the devices so that they do not complete at same time once the channel switching takes place. During the $\mathrm{CCH}$ interval short, high-priority application and system control messages are send. WAVE announcement frames are only allowed 
to be transmitted over the $\mathrm{CCH}$, while other management frames may use the $\mathrm{SCH}$. Both $\mathrm{CCH}$ and $\mathrm{SCH}$ can be utilized for regular data transfer. By default the device operates in $\mathrm{CCH}$. After the $\mathrm{CCH}$ interval expires, the $\mathrm{SCH}$ interval is activated and multimedia data is send during that interval followed by guard period and then the same cycle continues again. The total sync interval of $100 \mathrm{~ms}$ is allotted with $45 \mathrm{~ms}$ for each interval and $10 \mathrm{~ms}$ as two guard periods for one single synchronization cycle as shown above. The parameters of the traffic classes for each channel are shown in Table 2.3 and Table 2.4

Table 2.3: CCH Parameters.

\begin{tabular}{cccc}
\hline \hline ACCESS CATEGORY & CWmin & CWmax & AIFSN \\
\hline Voice(VO) & 3 & 7 & 2 \\
Video(VI) & 3 & 7 & 3 \\
Best Effort(BE) & 7 & 15 & 6 \\
Background(BK) & 15 & 511 & 9 \\
& & & \\
\hline
\end{tabular}

Table 2.4: SCH Parameters.

\begin{tabular}{cccc}
\hline \hline ACCESS CATEGORY & CWmin & CWmax & AIFSN \\
\hline Voice(VO) & 3 & 7 & 2 \\
Video(VI) & 7 & 15 & 2 \\
Best Effort(BE) & 15 & 511 & 3 \\
Background(BK) & 15 & 511 & 7
\end{tabular}

The above mentioned EDCA parameters that were used in $802.11 \mathrm{p}$ were carefully chosen. The logic behind chosing the smaller $\mathrm{CW}$ and AIFS for VO and VI was to give them priority on $\mathrm{CCH}$ as compared to $\mathrm{BE}$ and $\mathrm{BK}$ as these are life saving critical messages. The interesting thing to note here is that suppose VO and VI packets suffer from collision, then their CW would be doubled. Even after their $\mathrm{CW}$ have been doubled to $\mathrm{CW}$ max they would still have $\mathrm{CW}$ either equal to less than $\mathrm{BE}$ and $\mathrm{BK}$ which would still provide them priority over the lower AC. Moreover the data rates mentioned in Table 4.2 shows that VO and VI have higher data rate giving them supreme priority over other ACs. However on SCH if we compare the AIFS value for BE and BK its little 
smaller than AIFS value of $\mathrm{BE}$ and $\mathrm{BK}$ on $\mathrm{CCH}$, so we can say that $\mathrm{SCH}$ have milder EDCA parameters as compared to $\mathrm{CCH}$. As $\mathrm{BE}$ and $\mathrm{BK}$ needs to have priority on this channel we have provided them with higher data rate as mentioned in Table 4.2 to diminish the effect of EDCA parameters.

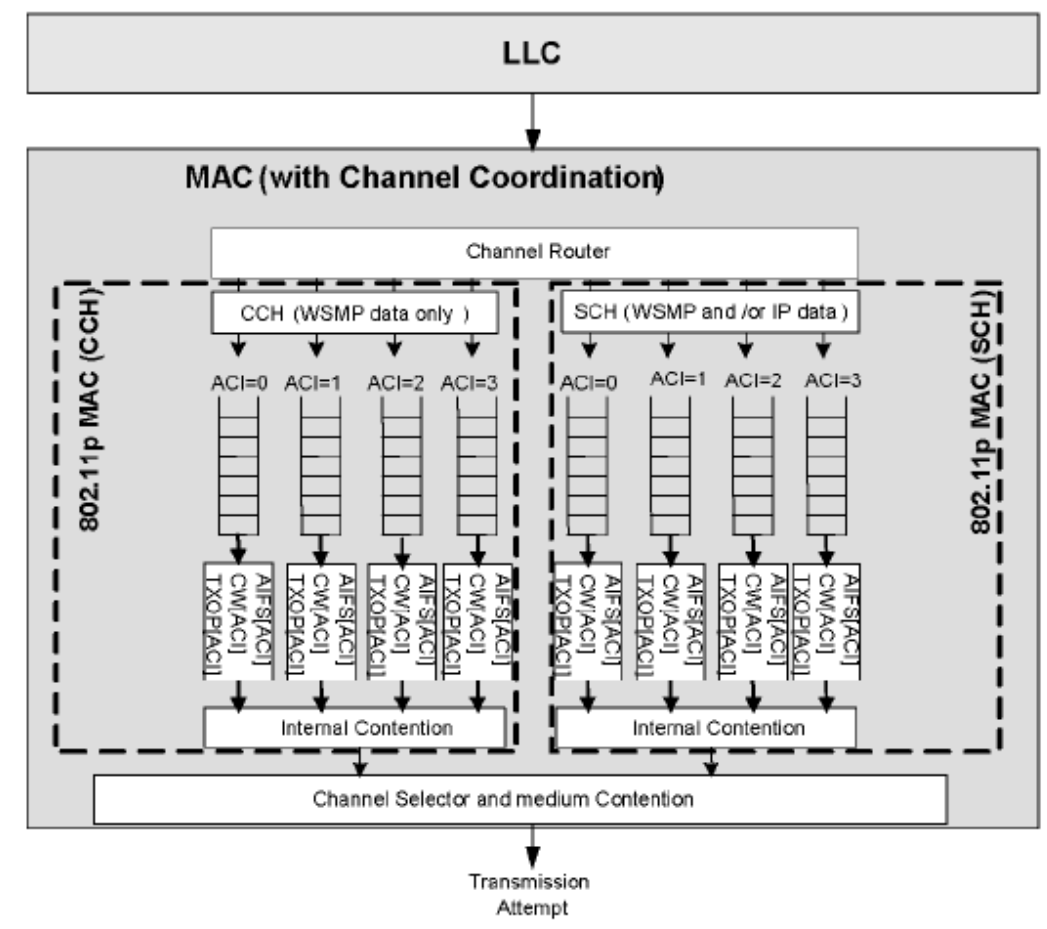

Figure 2.12: Channel Cooordination [33].

When the device works, either of these channels works and alternate switching takes place between them. Each channel has four independent queues, which have their own backoff process and different AIFS values and CW depending on the class they belong to. As each node has independent queues there might be a possibility that two queues might finish their backoff process at the same time. This unique case, the higher priority traffic goes through and the lower traffic priority behaves as if it suffered a collision and it performs a backoff process for the next backoff phase with its contention window doubled this is known as virtual collision. In our model, we do not consider virtual collision as we have only one type of data flowing in a particular device i.e if a 
device is wroking for $\mathrm{VO}$ it would only have $\mathrm{VO}$ data on $\mathrm{CCH}$ and $\mathrm{SCH}$ so there is no possibility of virtual collisions and hence we deal with external collision only.

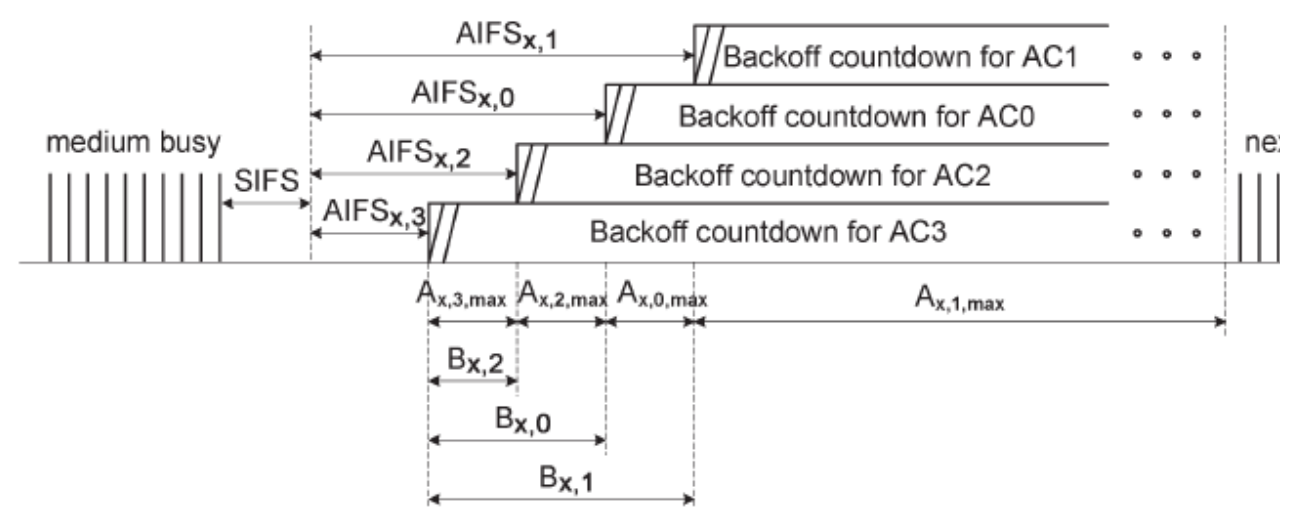

Figure 2.13: Channel Priority for IEEE 802.11p [31].

The Fig 3.5 above explains the priority mechanism in 802.11 p. If during the random backoff count, the medium becomes busy, then the counter would freeze and would start to decrement again only when the medium is found idle for SIFS + AIFS period of time. The counter that is used to count down the aifs value needs to be restarted everytime it is interrupted. Due to the synchronization with the end of the previous transmission, no access in possible for the highest priority AC i.e VO which has the lowest AIFS value. Also it can be concluded that the lower the priority of the $\mathrm{AC}$, highest the probability of it getting interrupted during the countdown as other ACs can start their countdown even when the lower priority ACs is still counting down. 


\section{Chapter 3}

\section{Simulator Design}

Artifex is a tool set for discrete event systems modeling, analysis and design. Artifex exploits a graphical language, which is based on Extended Petri Nets, to define detailed models of a system or process. Models can be executed on a simulator to obtain insight over the system under development, or to validate the model itself. At any stage of development, models can be automatically translated into $\mathrm{C}$ or $\mathrm{C}++$ code, which is then compiled into a single-process or multi-processes application, this allows quick development of prototypes, distributed real-time simulators, and distributed applications. The rectangular boxes that we have used are known as TRANSITIONS in which we have written the code for it to perform an action when an event occurs. The circular things are known as PLACE which passes the incoming TOKEN which carry the information and are used as storage to hold data units in the form of tokens. The fields of tokens are defined in the CMN units and values of variables can also be assigned to the fields of token and send it to measurements. We also have links whose main function is to connect the transitions and places. The important thing to note about transitions is that it can only fire when it has token to fetch from all the input places connected to it. The data can be transmitted between different classes with the help of interfaces as we can define the input and output places under an interface. The tokens are transmitted from one output palce of a class to another input place of the other class in the form of tokens which carry the useful simulation data. We can have multiple pages in a single class and also we can split a page into more pages. There are also a intial and final actions section in Artifex. In initial action we initialize the variables, which needs initial value at the start of the simulation. In final actions we can take measurement of the parameters. For our simulation model we have 
four main classes namely: Top Level Class which gives us the overview of the whole network in which devices are connected through medium to the RSU, and is used for taking the output values, Access Point which acts as a Road Side Unit(RSU), Medium which is used for transmitting data between devices and RSU and Device which implements the working of a On Board Unit(OBU).

\subsection{Block Diagram of Simulator}

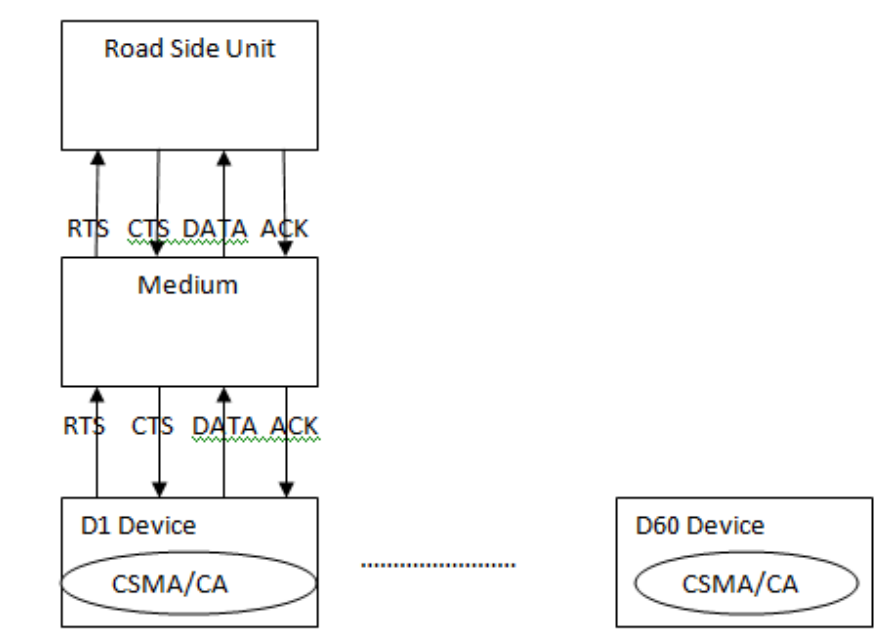

BLOCK DIAGRAM OF SIMULATOR

Figure 3.1: Block diagram of Simulator.

The diagramatic representation shown above gives the general view of how the simulator that we developed works. When the device completes its CSMA process and has RTS packet to send it goes through the medium to road side unit. In response to this RTS, the RSU sends a CTS to the same device from which it received the RTS. Once the device gets the CTS, data packet is send to the RSU by the same device and the RSU sends a ACK in response to complete the data transmission cycle. The following sections explain the design of the simulator which follow this 
flow of data transmission along with the other process associated with it.

\subsection{Top Level}

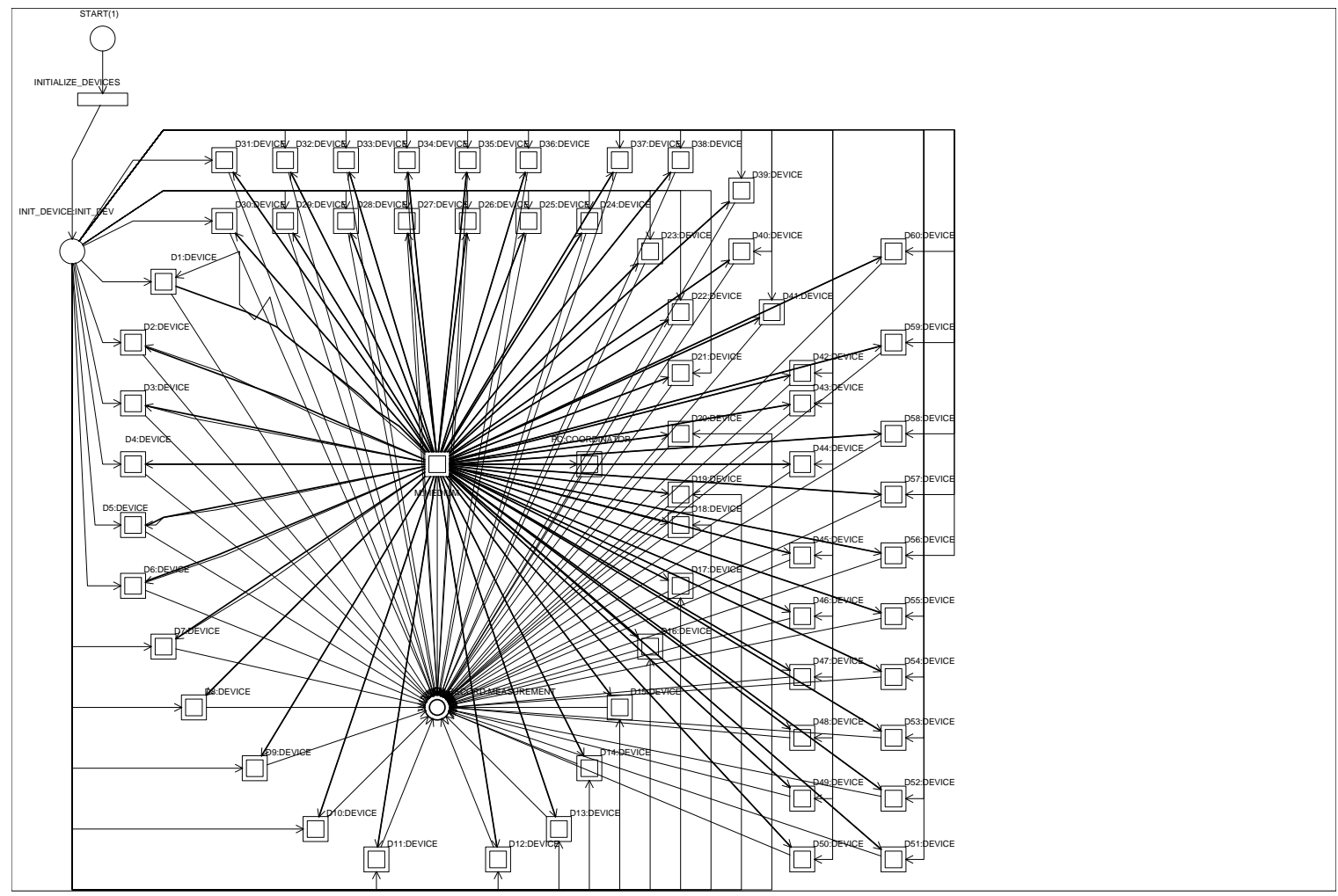

Figure 3.2: Top Level.

The simulator is initialized by the token from START place to INITIALIZE transition. This token is sent to all the devices which uses it to check the number of devices to be used and initializes them. TOP LEVEL is responsible to start the cycle of data transmission. In this model, the devices (D1, D2 till D60) transmits data to the RSU through the medium. The three entities: Devices, Medium, RSU are connected to the measurement page which contains all the counters and accumulators 
which are used for measuring the network parameters.

\subsection{Measurement}

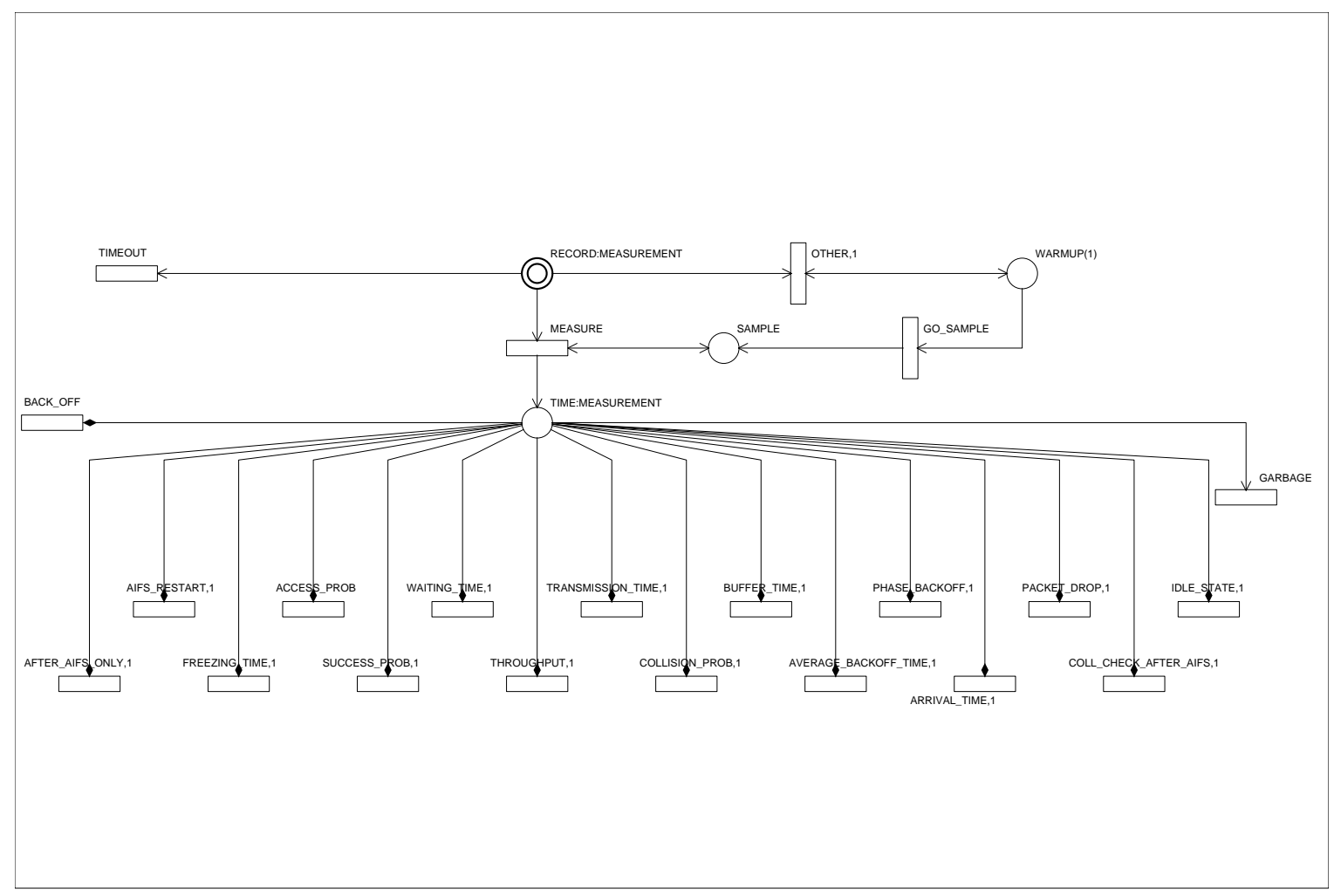

Figure 3.3: Measurement.

Each page in the device has a RECORD output place which is used to send the required values to the measurement page. Each device has an id which uniquely identifies itself in the network and thus the packet is send to the source and destination using this id. The RSU id is fixed as 0 . The traffic that flows is in both UPLINK and DOWNLINK direction. We have defined the complete cycle of the DATA packet flow from device to the RSU and back to the device through the medium for one packet. This is the pattern which is followed in this simulator for the DATA transfer. The 
transitions in the measurement page work by differentiating the type of measurement that is coming with its predicate and if the condition is satisfied, the counters are defined to measure the data for each specific class. Moreover, In the measurement page which used a delay so as to provide a sufficient amount of warmup time for the simulator before we start taking the actual simulation results. The figures above shows the two pages of Top Level. Fig 3.1 shows the overview of the network and how the devices are connected. Fig 3.2 represents the measurement page which is used to collect the output values from the simulator. 


\subsection{Road Side Unit}

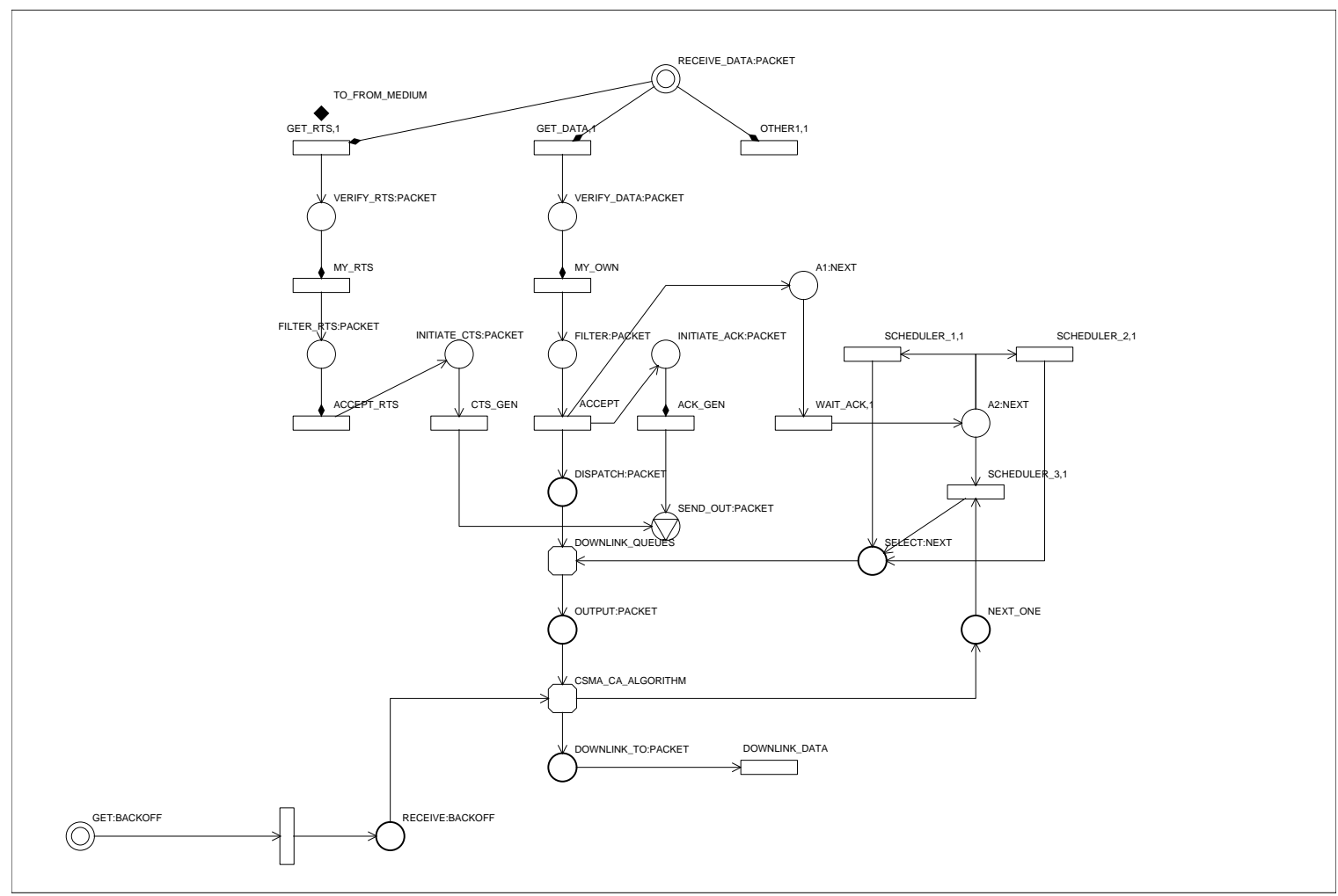

Figure 3.4: Road Side Unit.

When the RTS comes from the device through medium, the RSU checks the source, destination id and packet type $(\mathrm{CCH}$ or $\mathrm{SCH})$. The destination id should be 0 when the packet comes from the device because the RSU is assigned this unique id and the source id is the device id which is unique for every device. As RTS ( $\mathrm{CCH}$ or $\mathrm{SCH})$ depending on which channel is working is received by the RSU the CTS (CCH or SCH) is generated and sent to the device using the source id which was obtained when RTS was received to send it to the specific device. When the packet is send from the RSU the source id is 0 and destination is the device id, this information is embedded in the 
tokens which are transmitted. Similarly when data packet $(\mathrm{CCH}$ or $\mathrm{SCH})$ is received by the RSU, it sends $\mathrm{ACK}(\mathrm{CCH}$ or $\mathrm{SCH})$ back to the device using the same procedure for obtaining the source and destination ids to complete the data transfer cycle.

\subsection{Medium}

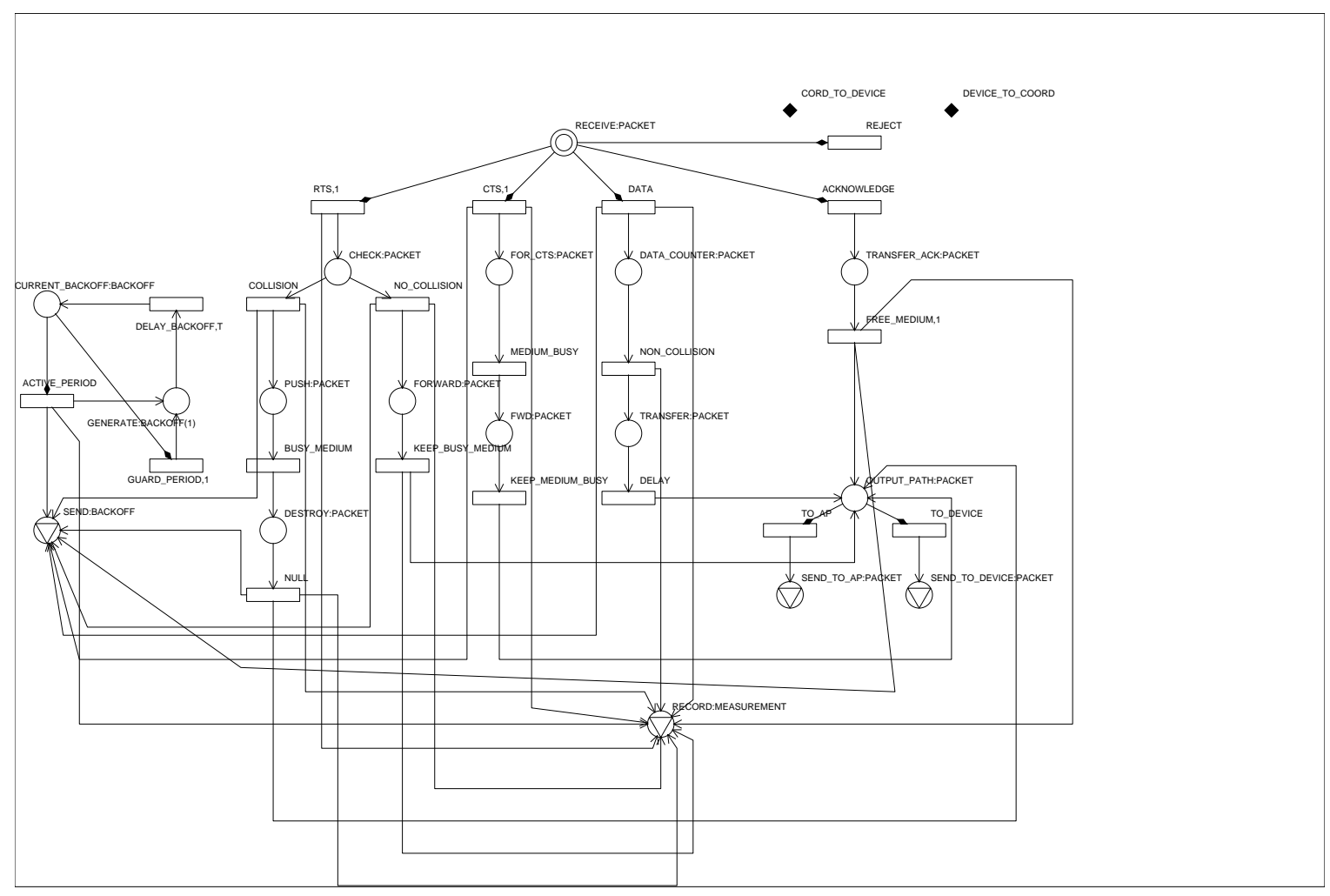

Figure 3.5: Medium.

UPLINK FLOW: The RTS (CCH or SCH) that comes from the device passes through the medium where it is checked for collision. If the medium receives more than one RTS at the same time from different devices, the transition COLLISION is triggered and the medium is kept busy for 
RTS_NAV period of time. After the collision with the help of the source id the packet is send back to the device to perform the next phase backoff with $\mathrm{CW}$ doubled as per the standard. In the other case, if there is no collision the RTS packet passes through the medium and at this moment the medium is kept busy for the RTS packet size period (in slots). The CTS (CCH or SCH) passes the medium in response to the $\mathrm{RTS}(\mathrm{CCH}$ or $\mathrm{SCH})$ followed by the data packet $(\mathrm{CCH}$ or $\mathrm{SCH})$ that comes in the medium, the medium stays busy for the period of data packet size and it is transmitted to the RSU.

DOWNLINK FLOW: When the required CTS $(\mathrm{CCH}$ or $\mathrm{SCH})$ comes in response to the RTS, it is forwarded to the device which is determined from the destination id field in the token and the medium is kept busy for the period of packet size of $\mathrm{CTS}(\mathrm{CCH}$ or $\mathrm{SCH})$ in slots. When the RSU gets the data packets $(\mathrm{CCH}$ or $\mathrm{SCH})$ it send the $\mathrm{ACK}(\mathrm{CCH}$ or $\mathrm{SCH})$ through the medium to the device. When the ACK $(\mathrm{CCH}$ or $\mathrm{SCH})$ is passing through the medium it stays busy for the ACK packet size but soon after it becomes IDLE after the ACK has been received for the next transmission to start. The other main function that the medium performs is to send the updated medium status. Whenever any packet is passing through the medium, it sends the BUSY status to the devices. If the medium is IDLE i.e no packet is being transmitted through the medium, the medium status is send as IDLE to the devices which results in the working of the processes (like AIFS or Random count decrement) in the devices. As the switching between the channels has to be done, the medium is used to perform this switching. When it is sending the medium status to the devices, we have information in our token as channel type which tells the device whether medium status is for $\mathrm{CCH}$ or $\mathrm{SCH}$. Once the duration of a particular channel is completed depending upon the duty cycle guard period follows as shown by the GUARD_PERIOD transition. This transition is triggered when one of the channels ends its cycle. As per the standard, no transmission should take place in this guard period but if a transmission is about to be completed it can use this guard period to complete this transmission and then the activity for the other channel starts. 


\subsection{Device}

\subsubsection{Initialize}

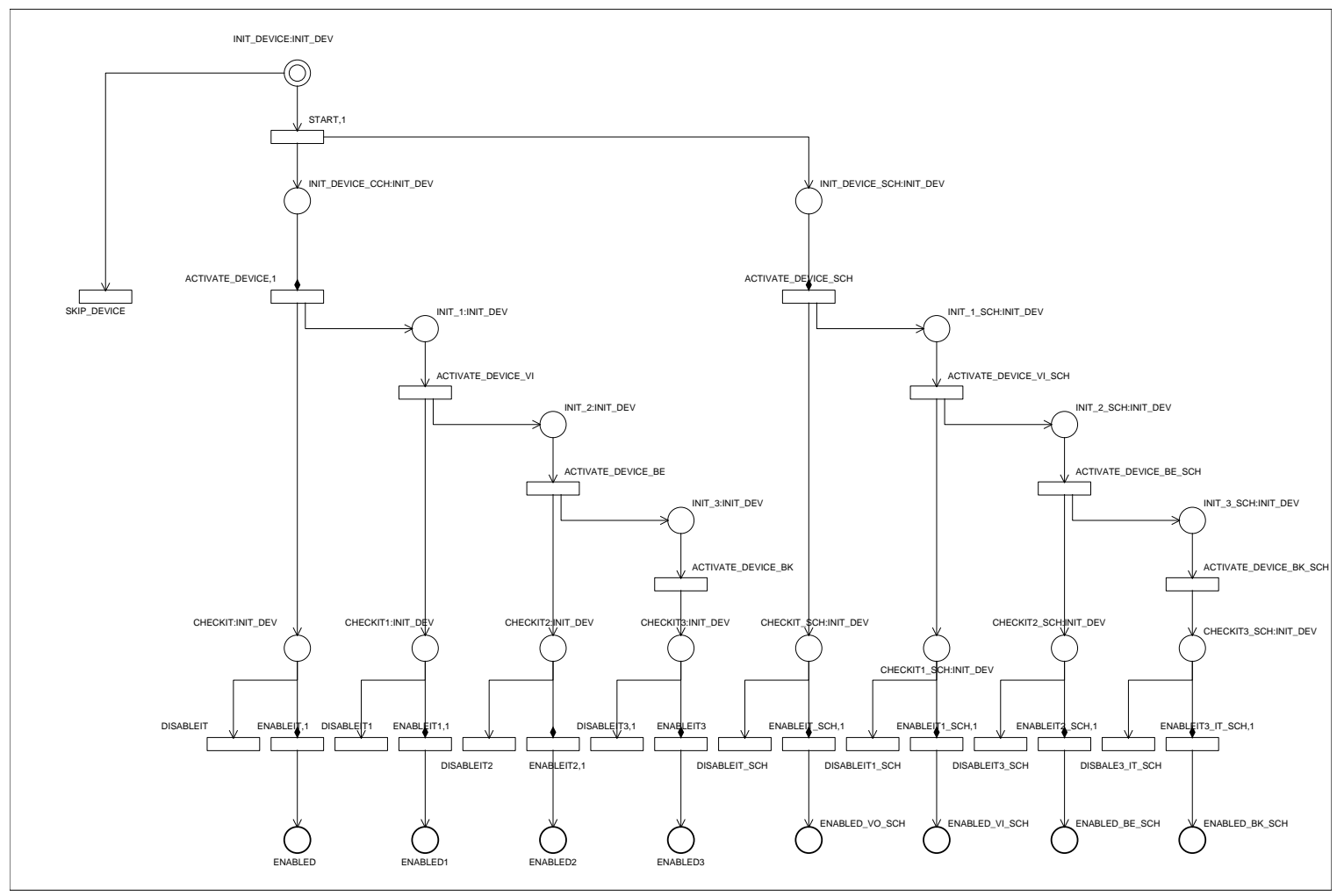

Figure 3.6: Initialize.

As the token comes in the INITIALIZE page, the transition START is used to copy the initial token to the INIT_DEVICE_CCH and INIT_DEVICE_SCH which enables the ACTIVATE_DEVICE and ACTIVATE_DEVICE_SCH transitions. Basically, these two transitions are used to initialize the devices. These two transitions checks the number of devices value contained in INIT DEV token send from the top level. If the number of devices assigned is not the same as assigned token goes into the SKIP_DEVICE which is checked by START transition. This page plays an important role 
as it assigns the arrival rate for each traffic class. If the device is assigned to work for any specific class it is assigned that arrival rate. Each device gets only single type of data ( $\mathrm{CCH}$ and $\mathrm{SCH})$ i.e if a device is working for Voice (VO) it would be activated only for Voice (VO CCH) and Voice (VO SCH). So with the help of code embedded in the transition VO is assigned 12kbps arrival rate and then this token is forwarded to the transition ACTIVATE_DEVICE_VI which also gets 12kbps. Further this token is transmitted to ACTIVATE_DEVICE_BE and ACTIVATE_DEVICE_BK which are assigned arrival rate $4 \mathrm{kbps}$ each. Also the population of the devices and for which class they would be working is done in these two transitions. The token is discarded if it does not belong to a specific class in DISABLE, DISABLEIT1, DISABLEIT2 and DISABLEIT3. The successful class sends the token to the place ENABLED, ENABLED1, ENABLED2, ENABLED3 which are merged in source poisson page to provide the inter-arrival delay for the generated packets using poisson arrival. The other part of this page also has the same working but it is for $\mathrm{SCH}$. It is used to perform the same funtion for $\mathrm{SCH}$ channel of the devices with the same working as explained above for $\mathrm{CCH}$. 


\subsubsection{Source Poisson}

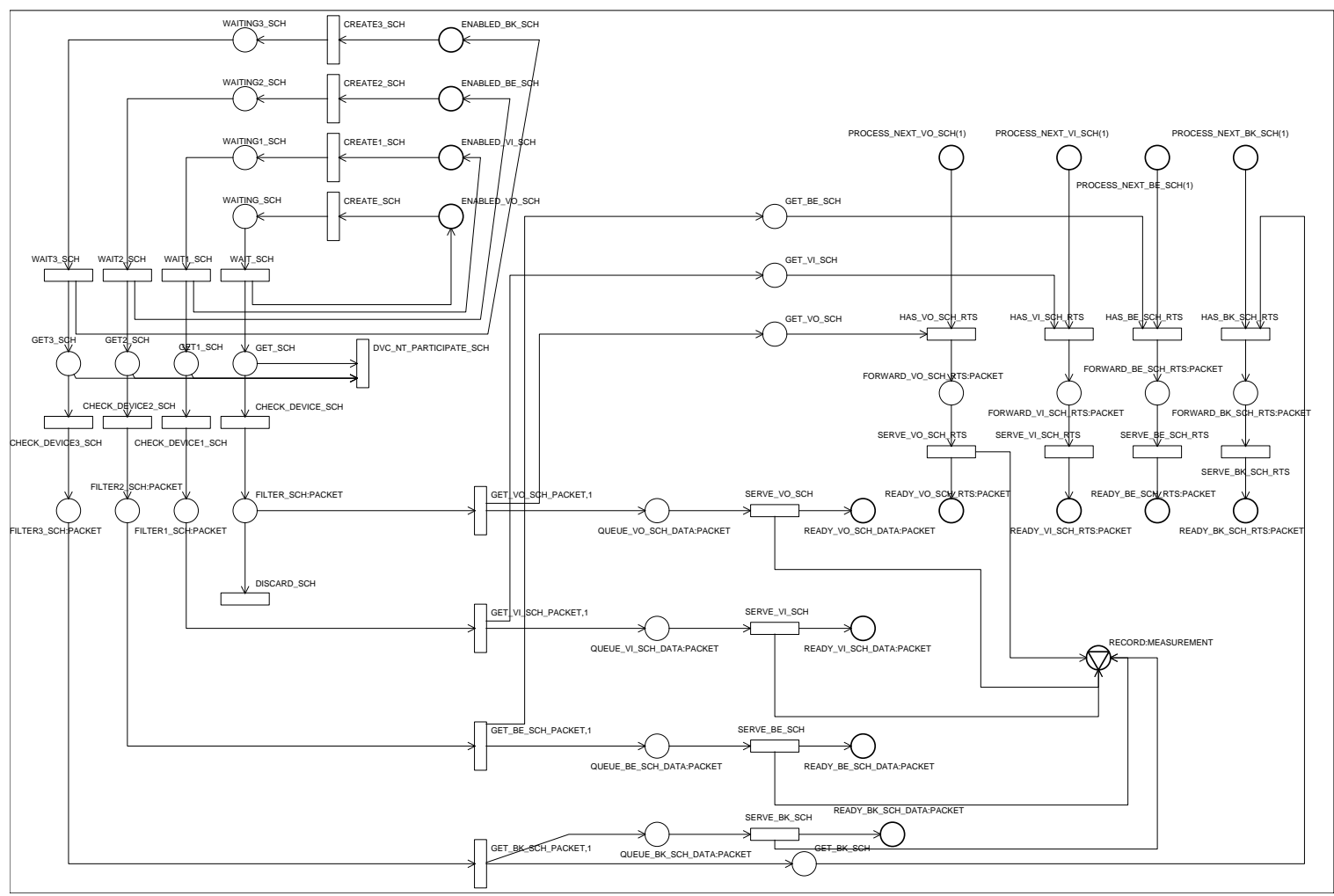

Figure 3.7: Source Poisson.

SOURCE POISSON PAGE: When the token comes to the ENABLED and other transitions depending on the traffic class and channel from the INITIALIZE page, the transition CREATE and other similar named transitions to produce a delay using the arrival rates assigned from top level leading to the interarrival time for the generated packets. As the token moves forward through various transitions and reaches GET_VO_PACKET and others the DATA packet is created. Along with the packet size, source id and other parameters that are to be used are set in GET_VO_PACKET transition. This token is stored in READY_VO_DATA and others which would fire only when CTS 
comes for it. Moreover these transitions which were used to generate DATA packets are send to HAS_VO_RTS and others to generate the required RTS packets along with packet size and source id. However this RTS packet would only be generated when the ACK for the previous transmission has been completed or ACK_TIMEOUT has occurred. Inorder to start the transmission for the first time initial tokens are provided to PROCESS_NEXT_VO and others which are also merged in the MAIN page of the device. The source and destination ids are also assigned at the time of RTS packet creation with source id being the station id which is used as a global parameter in the simulator and destination id being 0 as its being send to the RSU. For SCH also we have a similar page in design and code which has the same functionality as explained above. 


\subsubsection{CSMA/CA within EDCA}

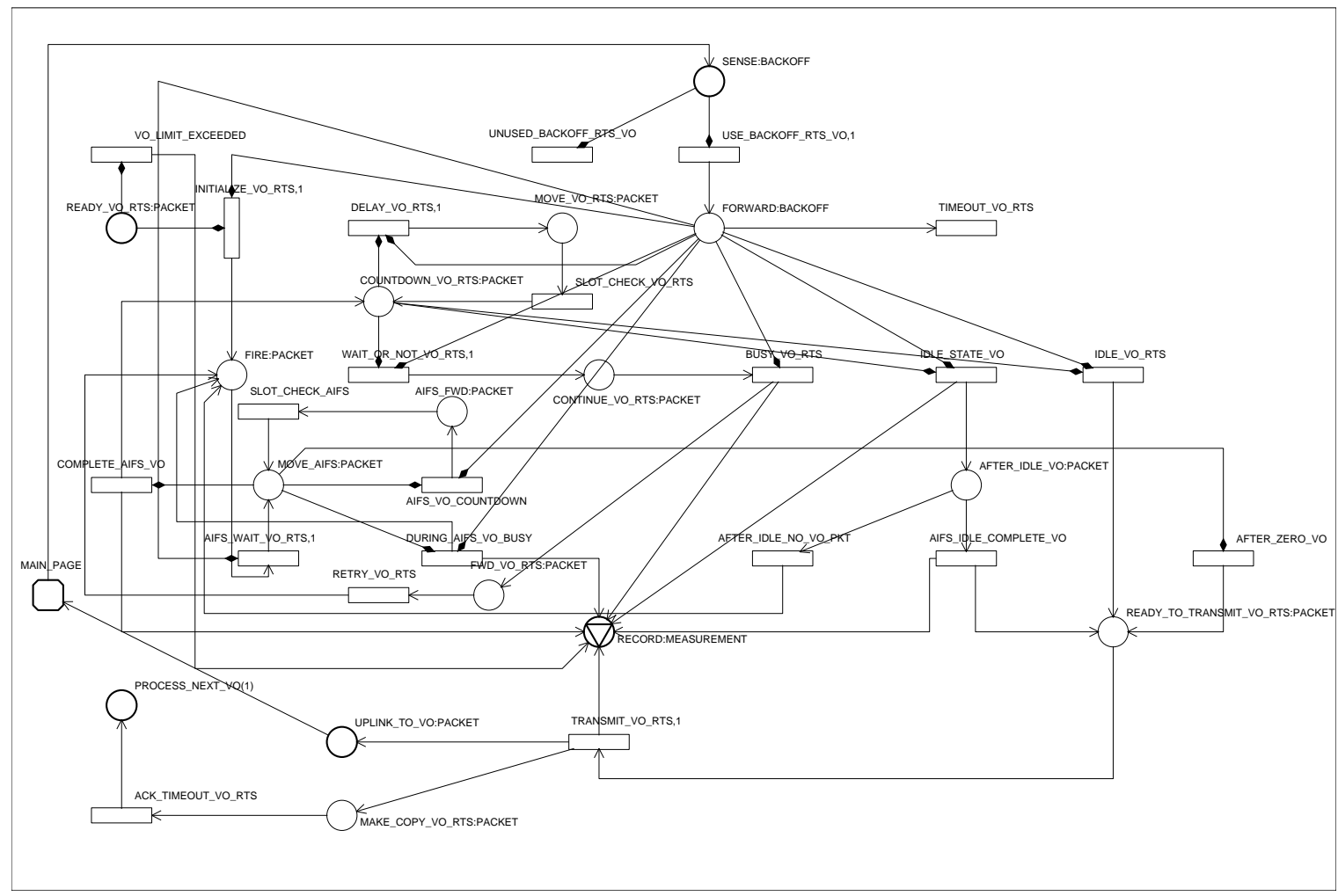

Figure 3.8: CSMA/CA within EDCA.

CSMA/CA: As the medium status is continuously coming to the device, the first thing that is checked is the channel type. If the $\mathrm{CCH}$ channel is working then the $\mathrm{CCH}$ backoff would be coming in the csma page. However when $\mathrm{SCH}$ channel is working the specific class csma would work and the other cch csma won't work and transition UNUSED BACKOFF consumes the tokens during that period leaving the csma page inactive for this period of time. When the token goes to the READY_VO_RTS and other similar named transitions for other data, the CSMA/CA process starts for each specific class. Here we would be explaining the working of VO CSMA/CA 
process because all the other csma would be working in the similar manner except the AIFS and $\mathrm{CW}$ value which are different for each class $(\mathrm{CCH}$ or $\mathrm{SCH})$ and have been specified in the later sections. The token goes to INITIALIZE_VO_RTS where it is assigned the contention window depending upon the phase. If it is the initial phase i.e phase 0 minimum contention window is assigned. If the phase number is greater than 0 then $\mathrm{CW}$ is assigned accordingly i.e it doubles till it reaches the maximum retry limit or maximum contention window limit. Also the token checks whether collision occurred or the previous transmission was successful. If the buffer is empty after successful transmission the zeroth backoff is performed. The token goes to AIFS_WAIT_VO_RTS where it is assigned the AIFS + SIFS value. The loop represents the AIFS countdown with every IDLE slot the counter decreases until it becomes 0. As during this countdown the updated medium status is received the counter only decreases when medium is IDLE. If AIFS becomes zero the packet goes to COUNTDOWN_VO_RTS for decrementing the random count. The random count is calculated with the help of CW which is assigned in the INITIALIZE_VO_RTS transition itself. If random count becomes 0, IDLE STATE is reached. If at this moment a packet is there in the buffer transmission takes place straightaway through AIFS_IDLE_COMPLETE_VO otherwise the packet waits for the other packet to come in the buffer and then it goes to this AIFS loop again and sends it after that through AFTER_ZERO_VO. After successful completion of csma process the packet goes to the MAIN page through UPLINK_VO from where it goes to the medium. All the other CSMA page for $\mathrm{CCH}$ and $\mathrm{SCH}$ works in similar fashion with different AIFS and $\mathrm{CW}$ values. 


\subsubsection{Device Main Page}

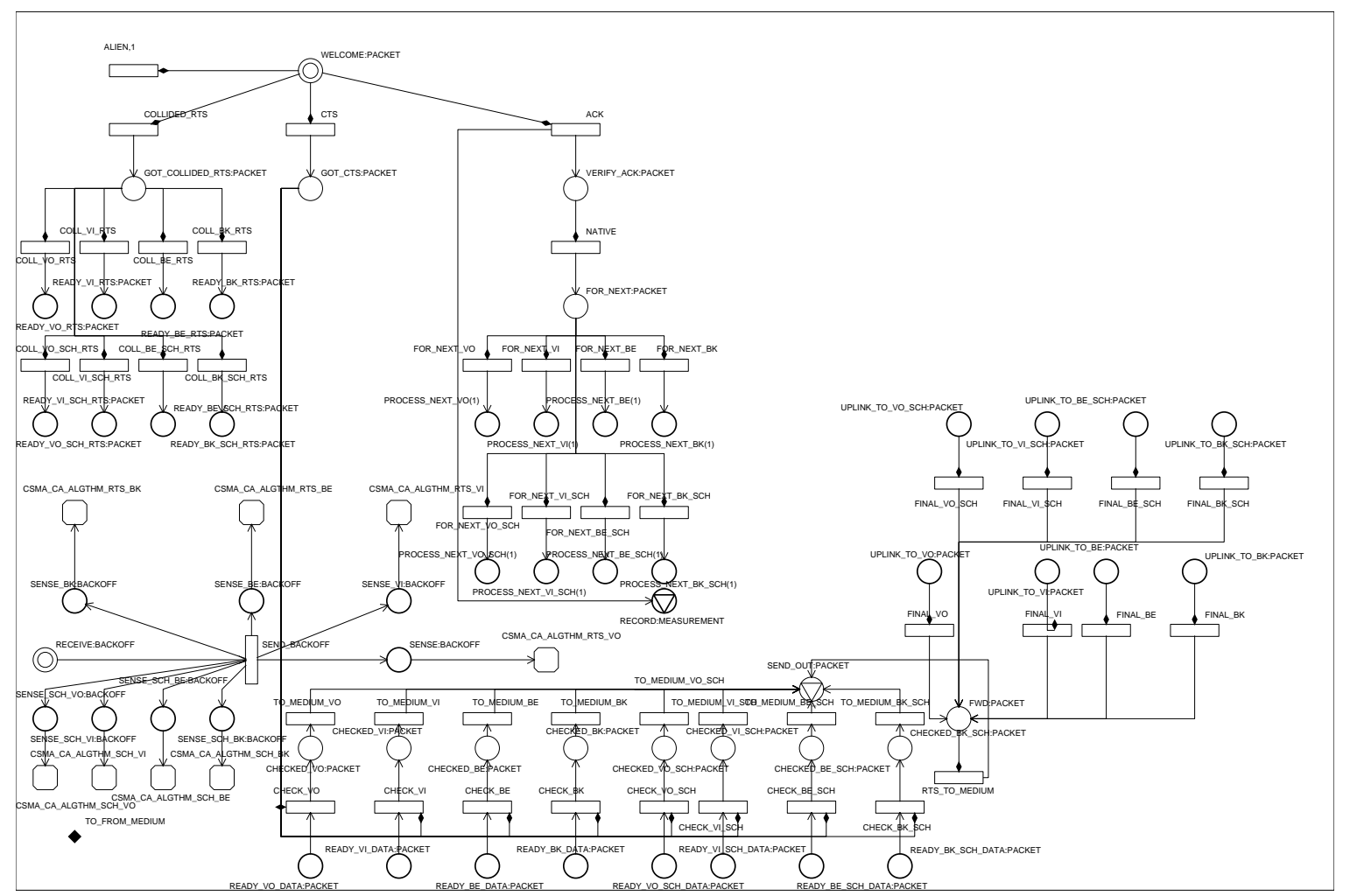

Figure 3.9: Device Main Page.

The main functionality of this main page is that it is used to send all the data that a device needs to send whether it is any management frame or any data frame. It accepts all the packets that a device receives from the access point and classify it according to the class it belongs to after which the next event starts. When RTS packet completes the csma process it goes to the main page of the device class depending on the specific class of RTS being send like VO would go to the transition FINAL_VO and then to RTS_TO_MEDIUM transition which is commom for all the traffic classes to forward it to place SEND_OUT which sends it out to the medium class for transmis- 
sion. However if collision occurs in the medium the packet comes back to the device main page as we change the source and destination id in the medium of the packet in case of collision so that the packet comes back to the device which had send it to the medium. When the packet comes back, the phase number is increased in COLL_VO_RTS transition and likewise for all the other traffic classes in the respective transitions and then again send to READY_VO_RTS for the csma process to start again with double contention window. However if the RTS is transmitted successfully through the medium, the device waits for the specific CTS in return from the RSU. When the CTS comes back to the device main page it goes to CTS transition whose predicate checks whether it CTS is of one the the class. After that it goes to CHECK_VO or any other similar named transition depending on the class of data being transmitted. The DATA packet which was stored at READY_VO_DATA or other similar transitions goes through to the medium through CHECK_VO annd after that TO_MEDIUM_VO. The noticeable thingis that transition CHECK_VO would only be triggered only when the CTS and DATA both are available for that specific class. After this ACK comes for the specific class which is differentiated by the transitions FOR_NEXT_VO and others using the predicate and sends it to PROCESS_NEXT_VO and others to trigger the next cycle for the transmission as the next RTS would only go to the transmission is complete or the packet is dropped. This main page has the same working for all the other classes and the SCH classes which is done with the help of information contained in tokens being transmitted as packets. the other important functionality of this page is that it is used to transfer the updated medium status which it gets from medium to the CSMA pages for different traffic classes as RECEIVE palce gets the token of BACKOFF which has updated medium status and the transition SEND copies this token to SENSE_VO and other similar named transition to make sure that all updated information is send to the CSMA page which has a reference on this page as CSMA_CA_ALGTHM_RTS_VO and others. 


\section{Chapter 4}

\section{Performance Evaluation and Analysis of}

\section{Results}

\subsection{Simulation Parameters}

IEEE 802.11p MAC layer is similar to 802.11e enhanced distributed channel access(EDCA) without the TXOP option. To investigate what happens to the performance parameters of the network, we would increase the population of the devices by 5 . As we increase the devices we observe that for $\mathrm{VO}$ and VI the increase in device is by 1 for every interval. However when we increase it for $\mathrm{BE}$ and $\mathrm{BK}$ the number of device that increases for BE also 1 but for BK it increases by 2 . The specific details for the population are given below in table 4.1. The legend notification is shown in table 4.2 and the data rate used by different $\mathrm{AC}$ in $\mathrm{CCH}$ and $\mathrm{SCH}$ in mentioned in table 4.3.

Table 4.1: Node Population.

\begin{tabular}{cc}
\hline \hline ACCESS CATEGORY & POPULATION \\
\hline Voice $(\mathrm{VO})$ & $0.2 *$ Population \\
Video(VI) & $0.2 *$ Population \\
Best Effort $(\mathrm{BE})$ & $0.3 *$ Population \\
Background $(\mathrm{BK})$ & $0.3 *$ Population
\end{tabular}


Table 4.2: Data Rate Used.

\begin{tabular}{ccc}
\hline \hline ACCESS CATEGORY & CCH DATA RATE & SCH DATA RATE \\
\hline Voice(VO) & $12 \mathrm{Kbps}$ & $12 \mathrm{Kbps}$ \\
Video(VI) & $12 \mathrm{Kbps}$ & $12 \mathrm{Kbps}$ \\
Best Effort(BE) & $4 \mathrm{Kbps}$ & $20 \mathrm{Kbps}$ \\
Background(BK) & $4 \mathrm{Kbps}$ & $20 \mathrm{Kbps}$ \\
& & \\
\hline
\end{tabular}

\begin{tabular}{cc}
\multicolumn{2}{c}{ Table 4.3: Legend Notations. } \\
\hline \hline LEGEND & NOTATION \\
\hline Voice(VO) & box \\
Video(VI) & asterisk \\
Best Effort(BE) & circle \\
Background(BK) & cross \\
& \\
\hline
\end{tabular}

The slot duration is 16 us and the packet size is 500 bytes. The size of the RTS packet is 6 slots, CTS is 5 slots, ACK is 5 slots. The duration of SIFS is 2 slots and the AIFS values are mentioned below for each specific class. The data rate is $3 \mathrm{Mbps}$ and the warmup time is 100000 slots. Moreover we would observe the effect of switching between $\mathrm{CCH}$ and $\mathrm{SCH}$ on the performance parameters with a specified duty cycle. The results obtained were plotted with the help of Maple[3]. The main parameters that we would be measuring during our simulation are:

- Access Probability: It is the ratio of the number of time a device accesses the medium to transmit a packet over the total idle time of the network.

- Normalized Throughput: It is defined as the average rate of successful data packet transmitted over the network. The throughput can be measured in terms of bits per second (bit/s or bps), or data packets per second or data packets per time slot. In our model we measure it in terms of percentage from total raw data rate.

- Average Backoff Time: It is the average time taken to complete the backoff process by the device so as to succeed in gaining the access to the medium following the backoff process. 
- Average Backoff Phase: It is defined as the phase number which successfully transmits the packet over the medium.

- AIFSN Restart Probability: It is the ratio of the number of times the AIFSN counter restarts for a specific class over the total number of restart of AIFSN counter for all the classes in the network of a particular channel.

- No Collision Probability: It is probability of the data packet not colliding with any other packet in the network while undergoing transmission.

\subsection{Simulation Scenarios and Performance Evaluation of 802.11 DCF}

For DCF we performed the simulation as this was the first phase of my thesis and later on I extended this simulator to $802.11 \mathrm{p}$. In this I examined two scenarios to see the performance of the network.

- Simulated with different arrival rates and fixed number of devices.

- Simulated with different number of devices and fixed data rate. 


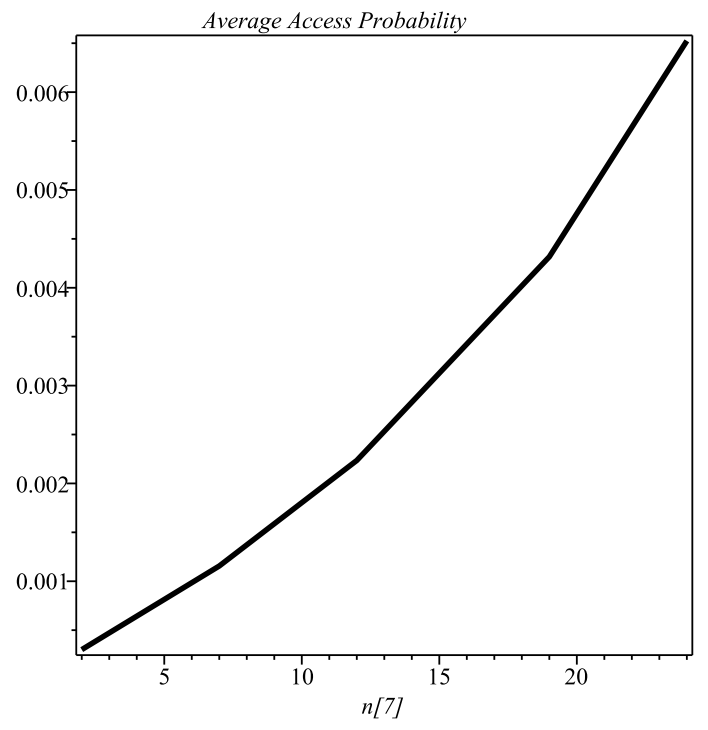

(a) Average Access Probability

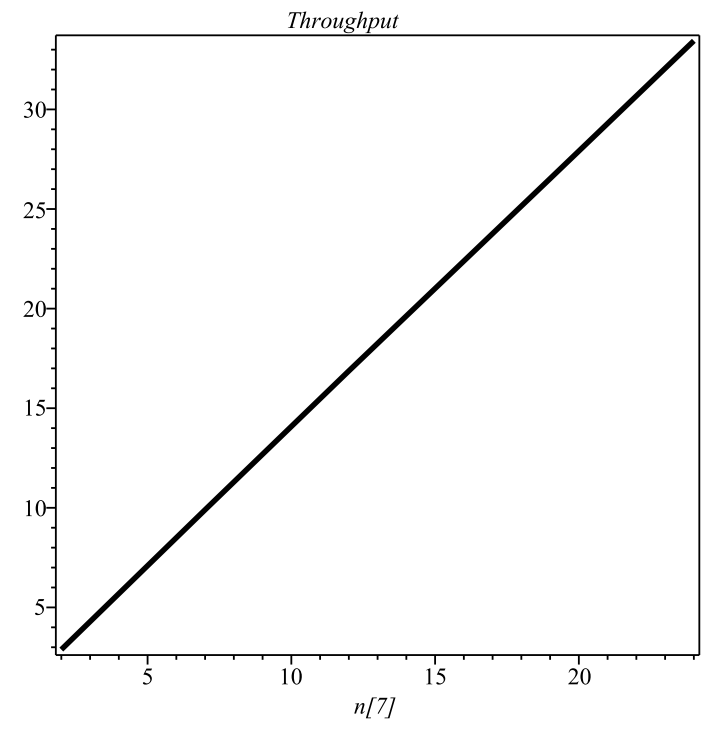

(b) Throughput

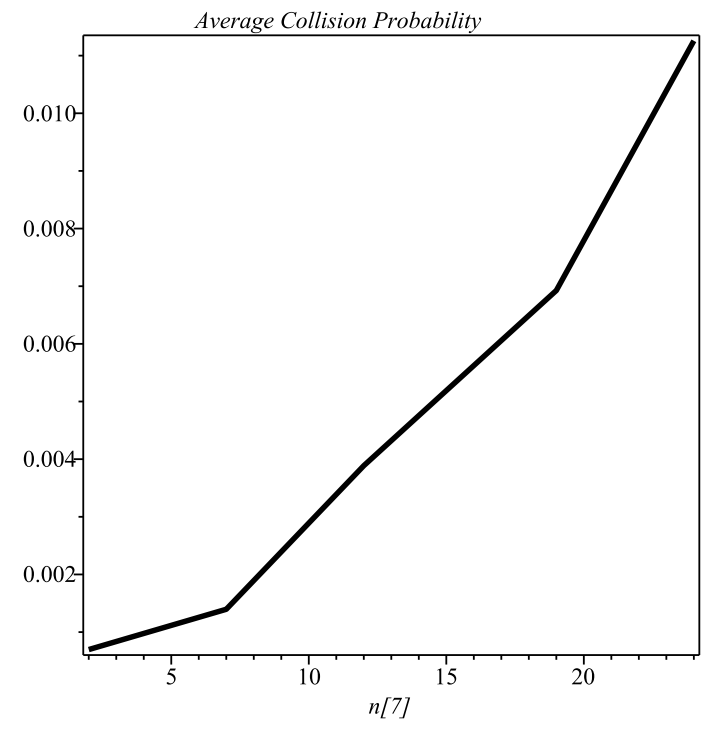

(c) Average Collision Probability

Figure 4.1: Average Access probability, Throughput, Average Collision Probability for different arrival rates and fixed number of devices. 


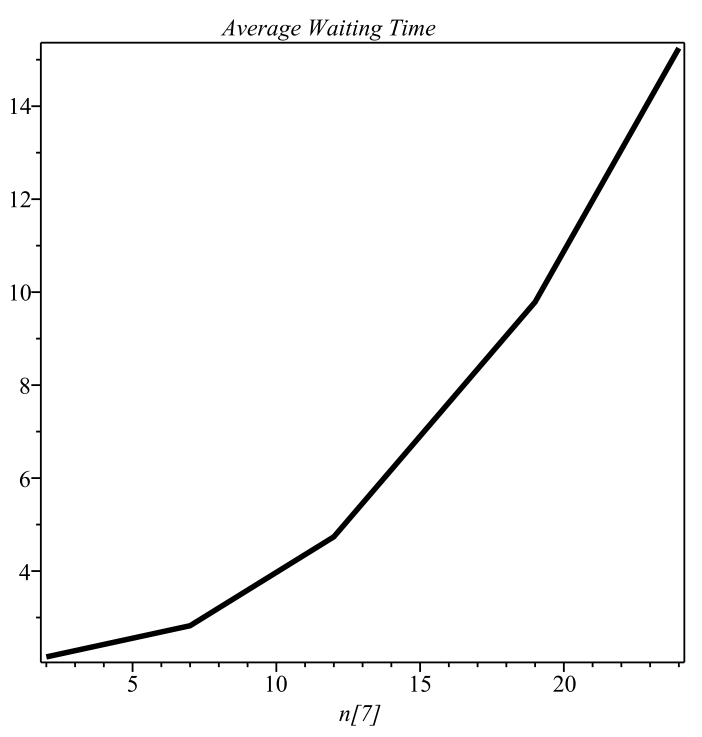

(a) Average Waiting Time

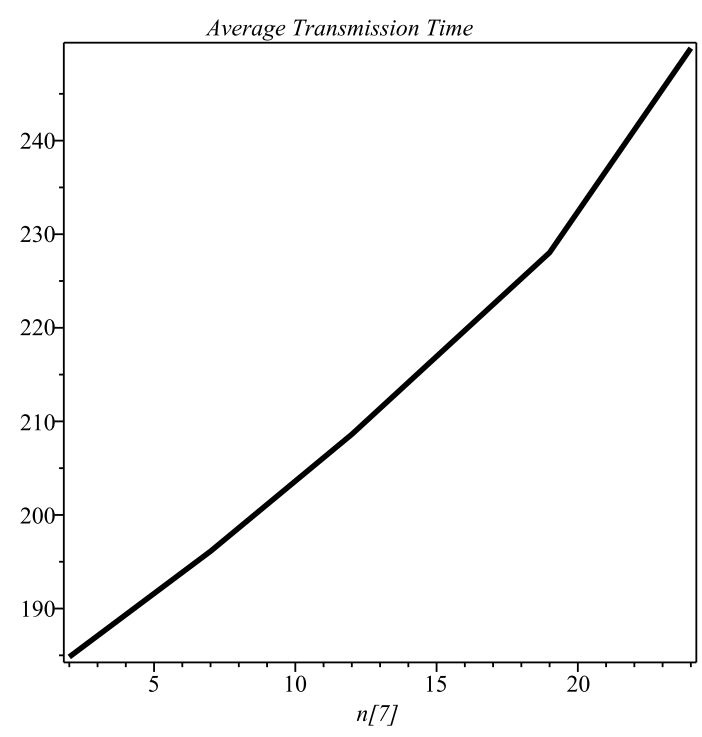

(b) Average Transmission Time

Figure 4.2: Average Waiting Time and Average Transmission Time for different arrival rates and fixed number of devices.

The scenario that we have examined above was that we had variable arrival rates and a fixed number of devices. We started by examining the throughput of the network which kept increasing linearly with the increase in the load as expected. Later, we increased the load further to observe its affect on the throughput. With increasing load the average access probability of the network also increases as the data would be transmitted faster because the devices would be accessing the medium more often. The average collision probability increases with the load as devices would be sending more data to the medium. As a result of increase in collision probability, the waiting time and transmission time of packet also increases as more attempts are made to send the packet. 


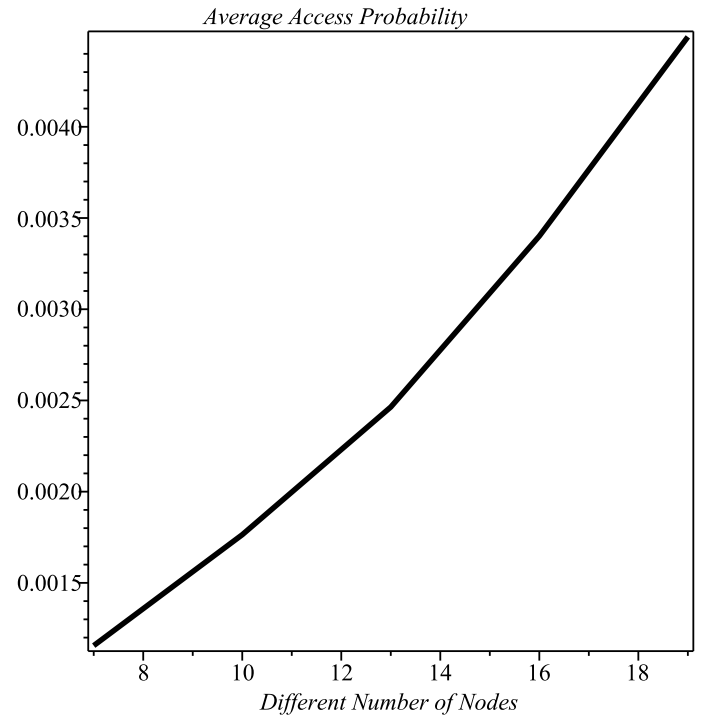

(a) Average Access Probability

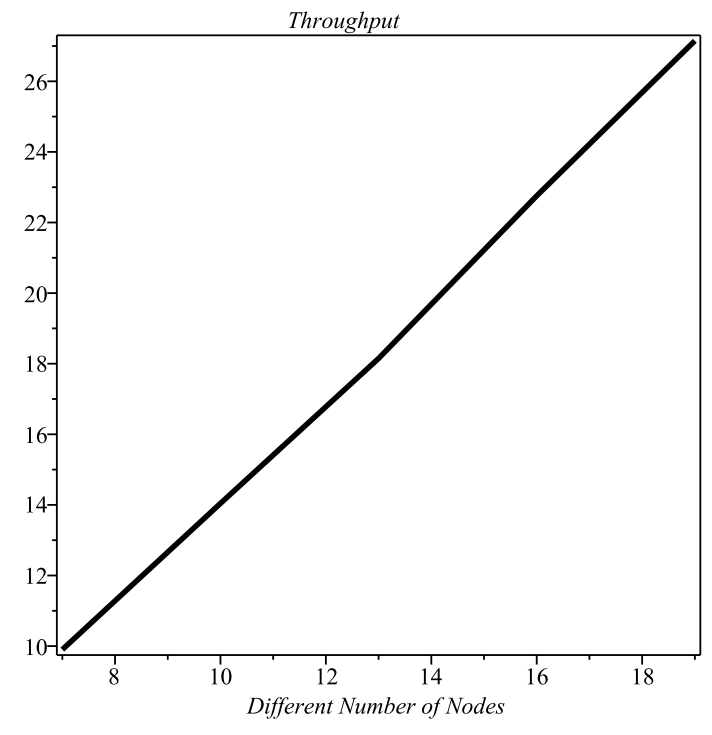

(b) Throughput

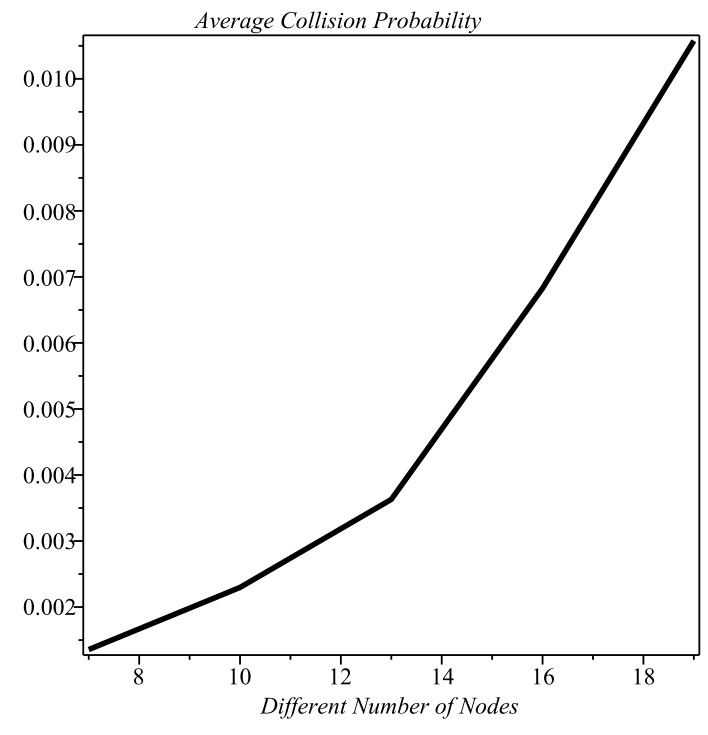

(c) Average Collision Probability

Figure 4.3: Average Access probability, Throughput, Average Collision Probability for different number of devices and fixed arrival rate. 


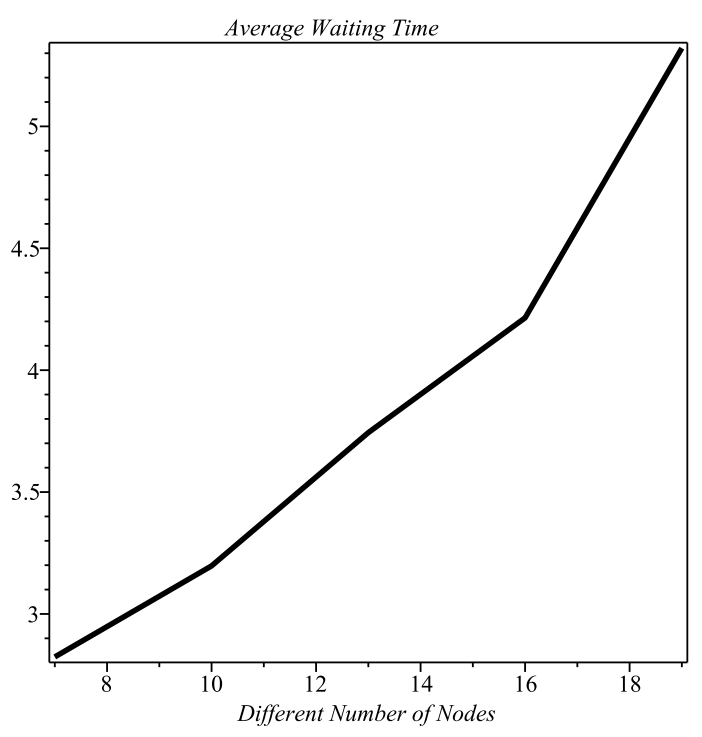

(a) Average Waiting Time

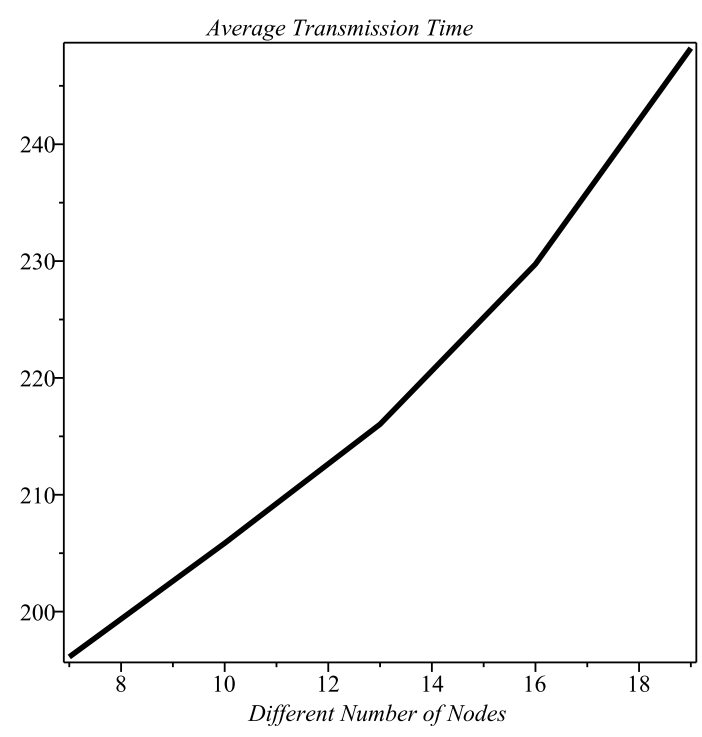

(b) Average Transmission Time

Figure 4.4: Average Waiting Time and Average Transmission Time for different number of devices and fixed arrival rate.

On examining this scenario where we are increasing the number of devices and a constant arrival rate, we observe that the throughput increased linearly with the increase in the number of devices. As the number of devices increased, they transmitted more data which increased the throughput. As throughput is increasing the average access probability would also increase because more devices would access the medium. With more devices, the probability of packet collision would increase as all of them would try to transmit and because of this the waiting time and the transmission time of the packets also increases as more attempts are made to send the same packet because of collisions. 


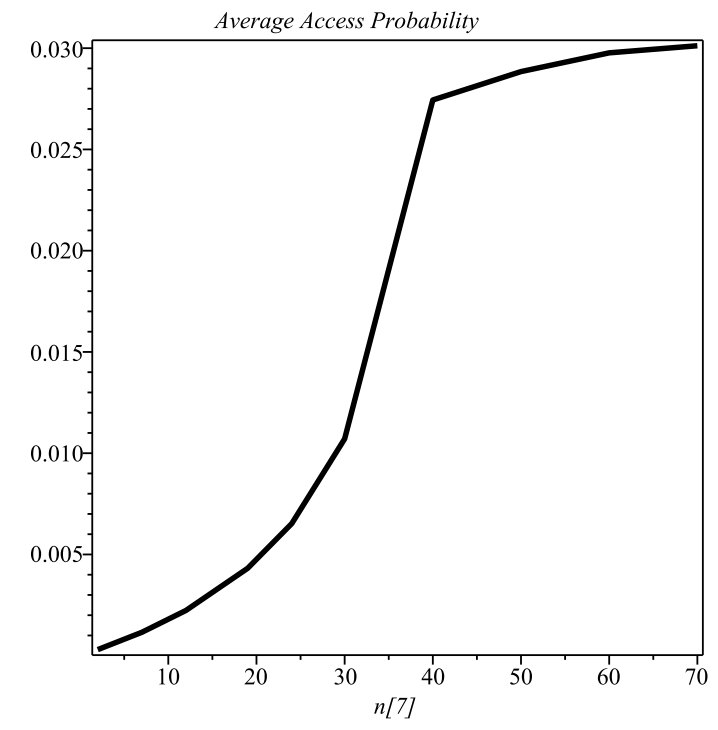

(a) Average Access Probability

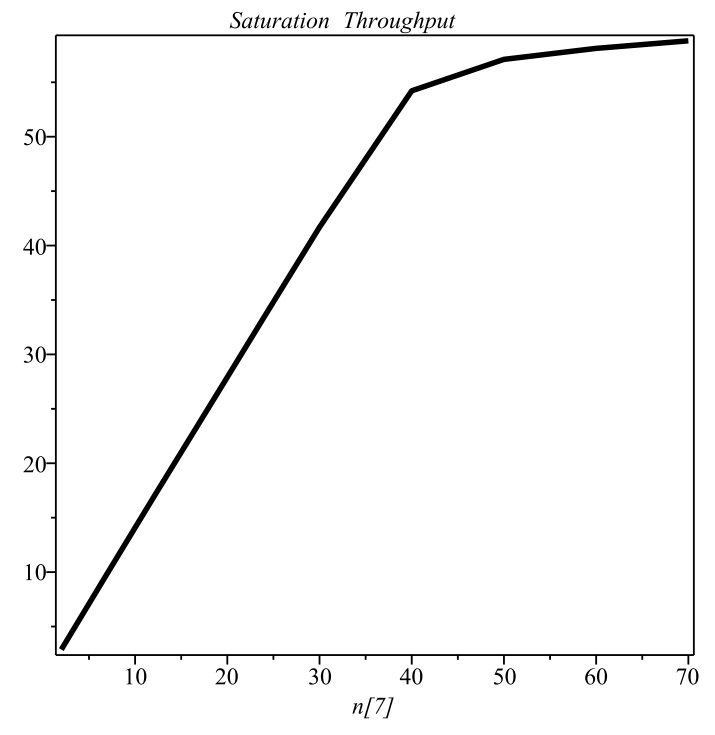

(b) Throughput

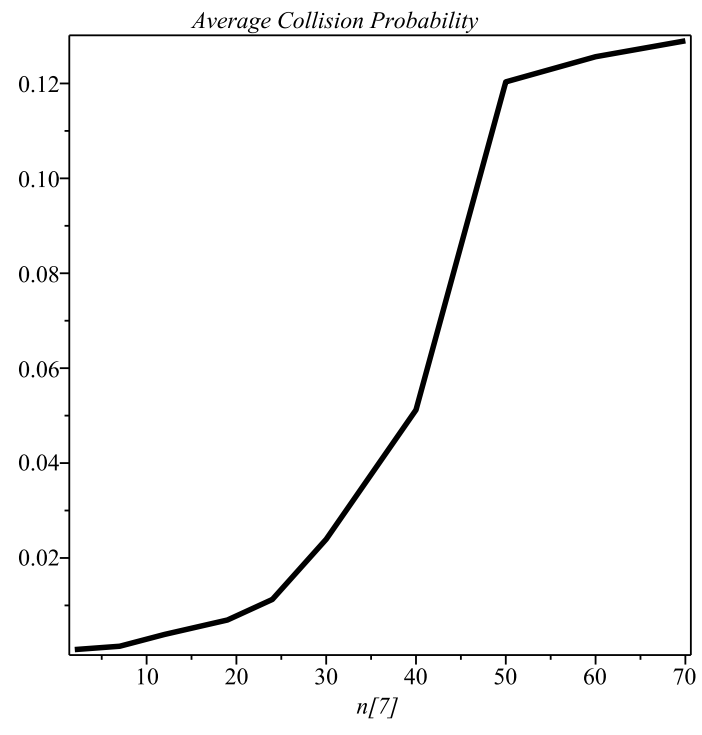

(c) Average Collision Probability

Figure 4.5: Average Access probability, Throughput, Average Collision Probability different arrival rates and fixed number of devices equal to 7 . 


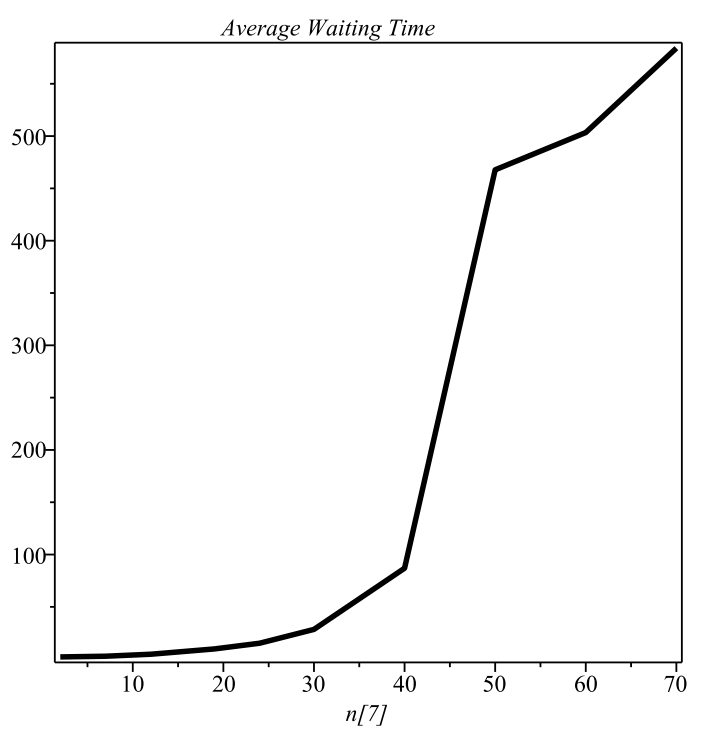

(a) Average Waiting Time

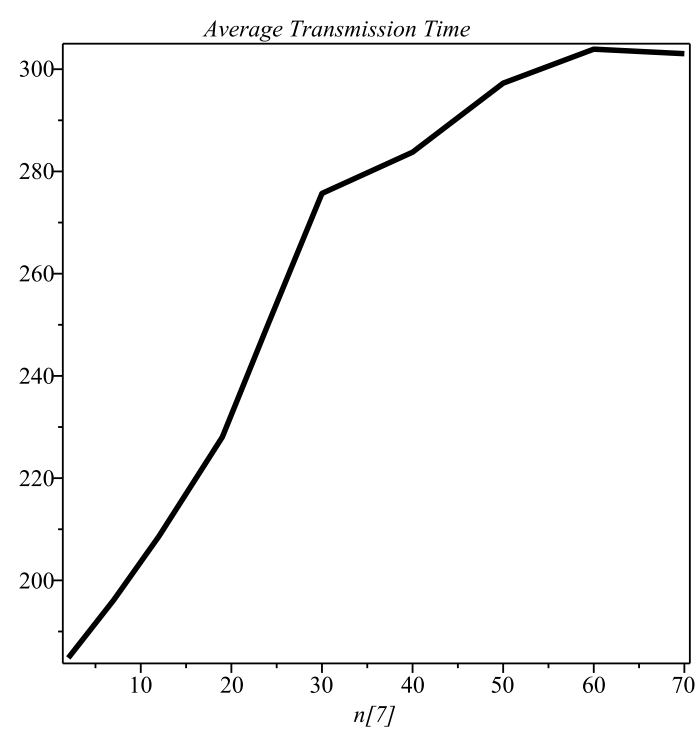

(b) Average Transmission Time

Figure 4.6: Average Waiting Time and Average Transmission Time for different arrival rates and fixed number of devices equal to 7 .

The other experiment that we have performed had a fixed arrival rate and different number of devices. We specifically chose high arrival rates upto 70 frames per second to observe how the network behaves in the saturation zone. With this high arrival rate, the network is bound to get into saturation. We started with this arrival rate for 7 devices and later increased the number of devices to study the trend. 


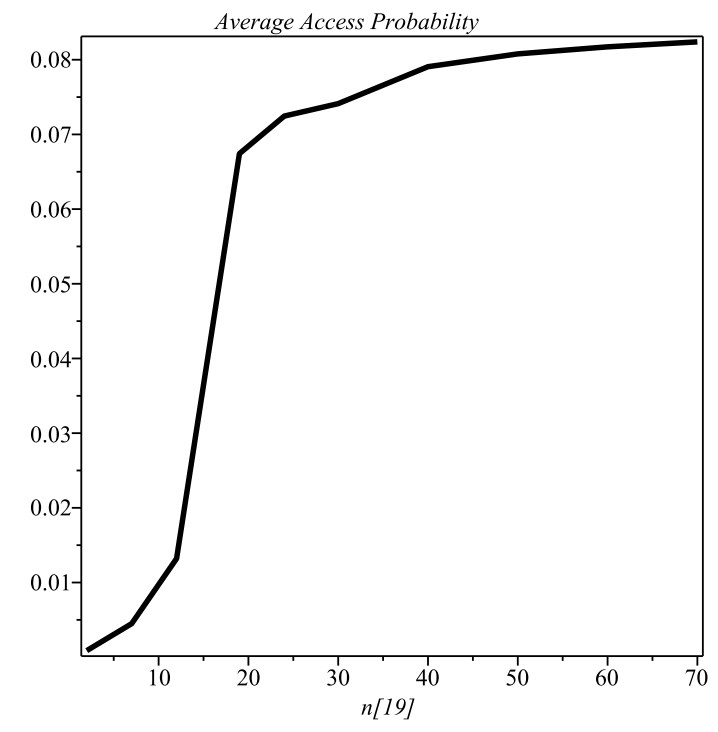

(a) Average Access Probability

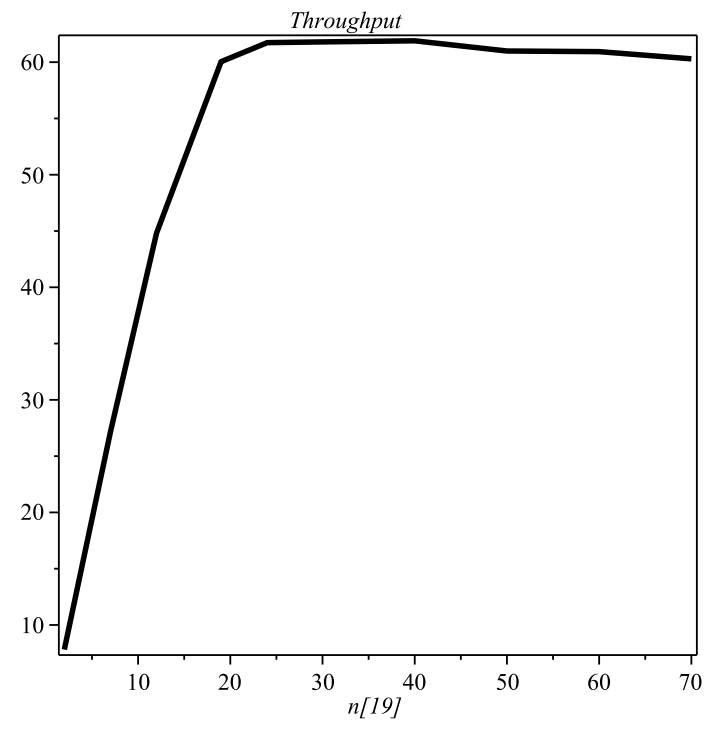

(b) Throughput

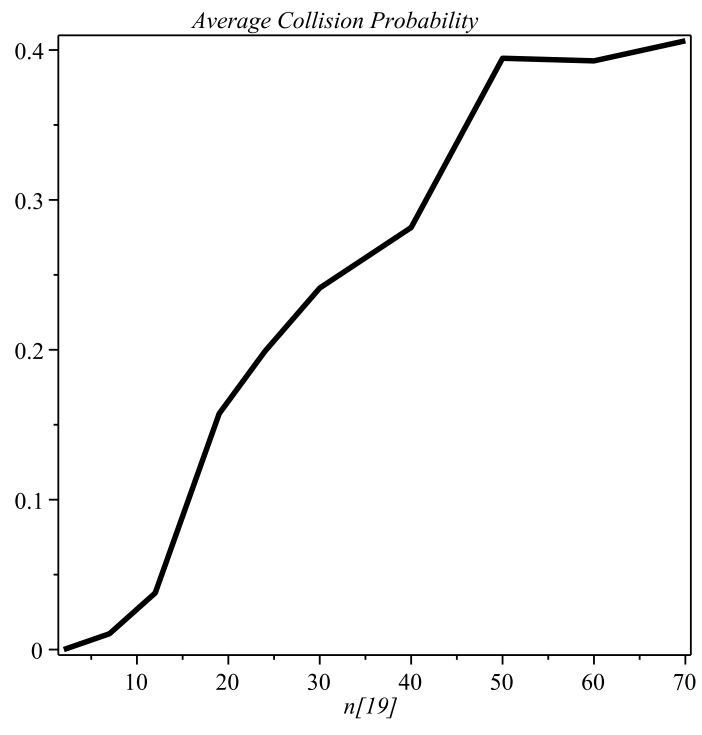

(c) Average Collision Probability

Figure 4.7: Average Access probability, Throughput, Average Collision Probability for different arrival rates and fixed number of devices equal to 19 . 


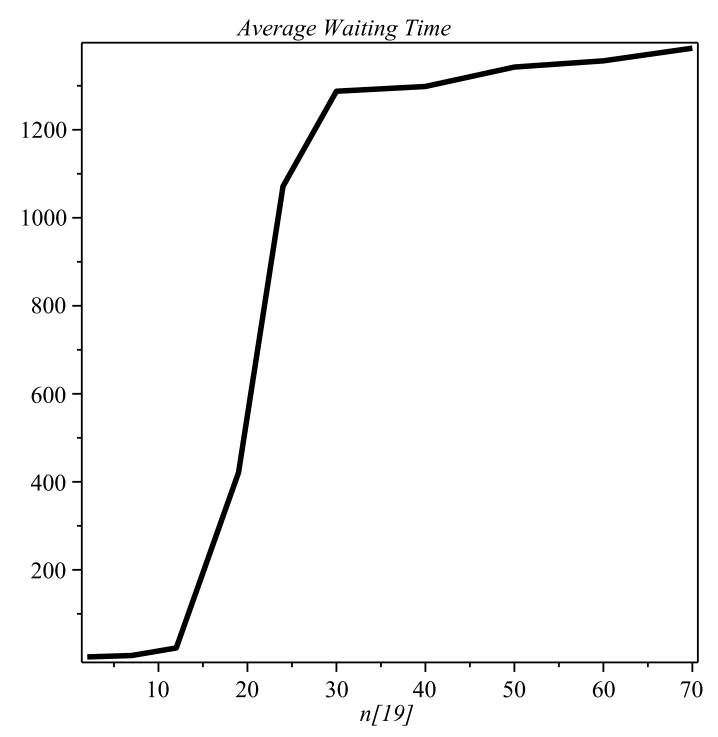

(a) Average Waiting Time

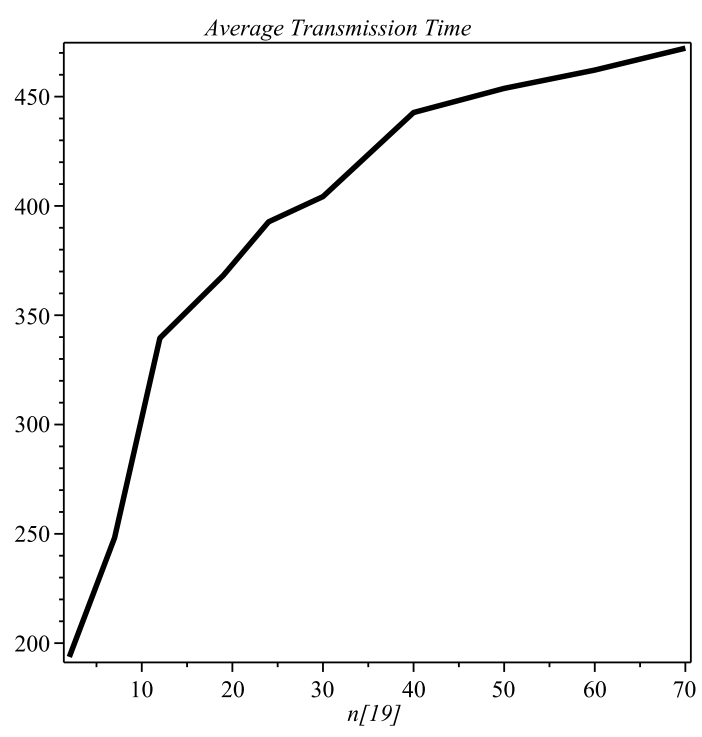

(b) Average Transmission Time

Figure 4.8: Average Waiting Time and Average Transmission Time for different arrival rates and fixed number of devices equal to 19 .

For both experiments we observe that the throughput instead of increasing begins to flatten as the network enters into the saturation i.e it starts to drop more packets than it can transmit, resulting in this type of behaviour. On increasing the number of devices to 19 , this behaviour is seen more clearly as the network condition deteriorates further with the increase in the number of devices. As expected, the average access probability will also begin to flatten as the devices would not be able to access the medium more often as more packets would be dropped than transmitted for both the cases. The flattening of the average collision probability confirms the fact that the network has entered into the saturation zone since the packets that are transmitted has been reduced. The average waiting time and transmission time show some flattening but still they are increasing in small values. 


\subsection{Performance Evaluation of 802.11e EDCA}

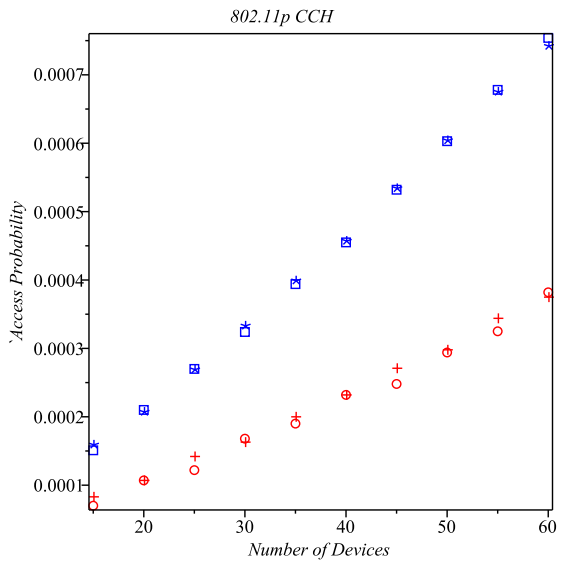

(a) Access Probability

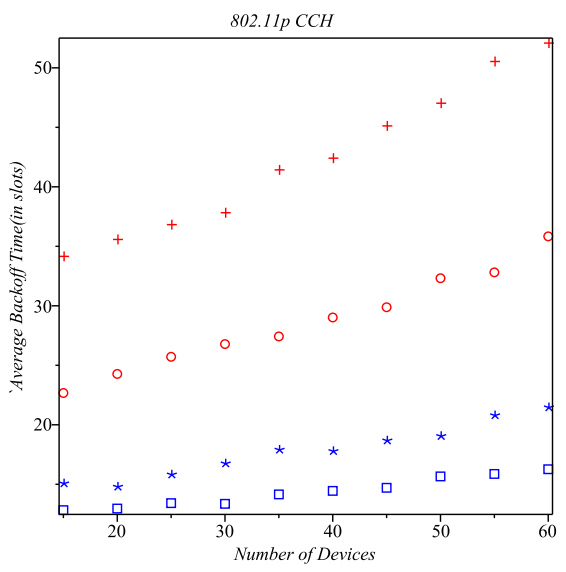

(c) Average Backoff Time

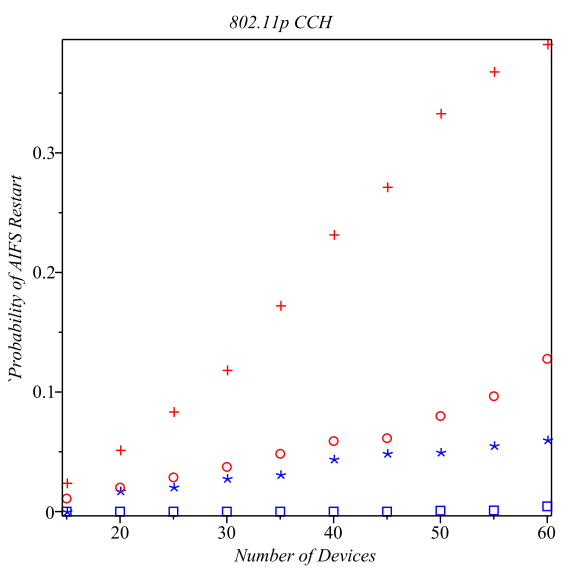

(e) AIFSN Restart

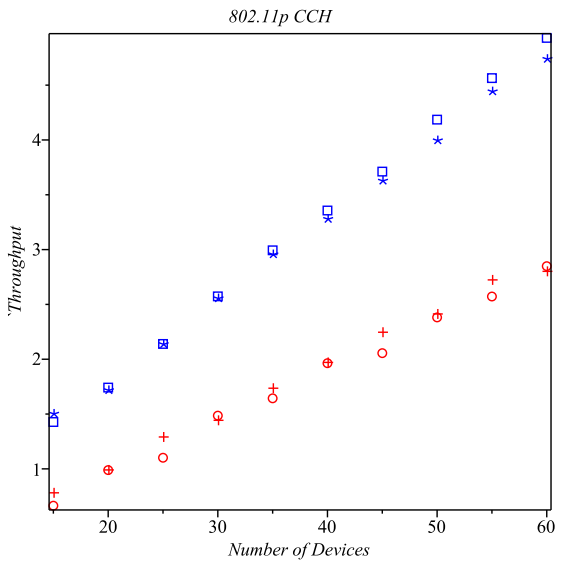

(b) Throughput

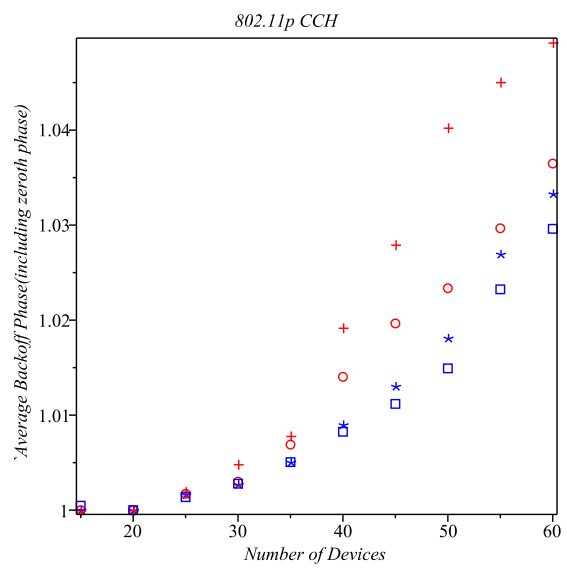

(d) Average Backoff Phase

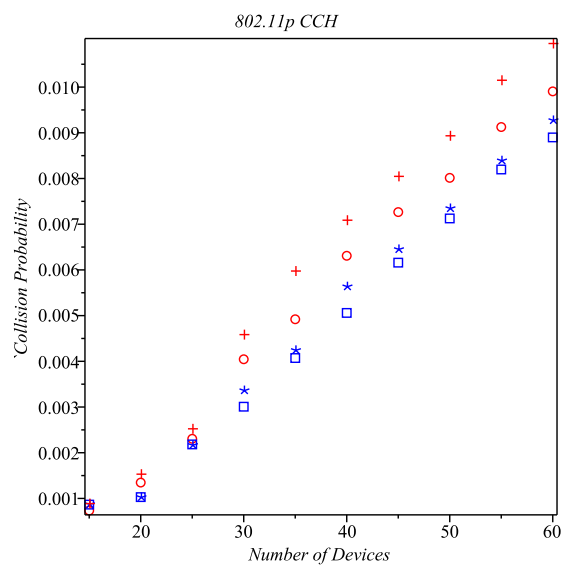

(f) Collision Probability

Figure 4.9: Performance Parameters for 802.11e EDCA. 
The parameters that we have used for this model are the default EDCA parameters mentioned in Table 2.2 and the data rates as shown in Table4.2. The parameters are similar to the parameters that are used in 802.11p CCH. Due to these parameters and QoS that could be used in 802.11p, we initially designed 802.11e. The results verify the fact that the difference in the graphs of different traffic classes is due to the AIFSN values and the contention windows i.e EDCA parameters. The throughput is maximum for the Voice (VO) and lowest for the Background (BK). VO is expected to have the highest throughput because it has the highest priority along with high data rate and the messages should be transmitted with maximum ease along with VI as compared to other ACs whose throughput is less than the higher priorities. This fact is further confirmed by the collision probabililty and AIFSN counter restart probability. As the BK class traffic is colliding the maximum, the throughput should be minimum for BK as we have obtained. The access probability for $\mathrm{VO}$ is the greatest along with VI because of the short AIFSN and contention window value as obtained in the above graph. The average backoff time is highest for the BK class because of the AIFSN and CW values. Moreover, as the AIFSN restarts most for it, the average backoff value is maximum for BK which takes the maximum time to complete the transmissions and lowest for $\mathrm{VO}$ as it has the highest priority, making transmissions with maximum ease and minimum time. Similarly because of this reason, the average backoff phase value also follows the same trend. BK has the worst performance because of the EDCA parameters providing it with lowest priority. VO has the shortest AIFSN value so it is never interrupted and because all the transmissions are synchronized the AIFSN counter never restarts for VO. The collision probability is maximum for the BK as expected because it is interrupted most often and its EDCA parameters gives it less chance to transmit whereas VO performs the best even in terms of collision. 


\subsection{Performance Evaluation and Analysis of Results for 802.11p With Different Duty Cycles}

In this work we performed experiments with different duty cycles to see the performance of $\mathrm{CCH}$ and $\mathrm{SCH}$ under varying factors like number of devices and different data rates for different ACs under different duty cycles. The duty cycle is varied with the help of SO which is regulated according to the $\mathrm{CCH}$. The duty cycle value for $\mathrm{SCH}$ is (1-SO). So the duty cycle for $\mathrm{CCH}$ of 0.6 has the maximum bandwidth with 0.3 having the least bandwidth and for SCH 0.3 has the highest bandwidth and 0.6 has the least bandwidth as we have total bandwidth of $3 \mathrm{Mbps}$ as per the standard.

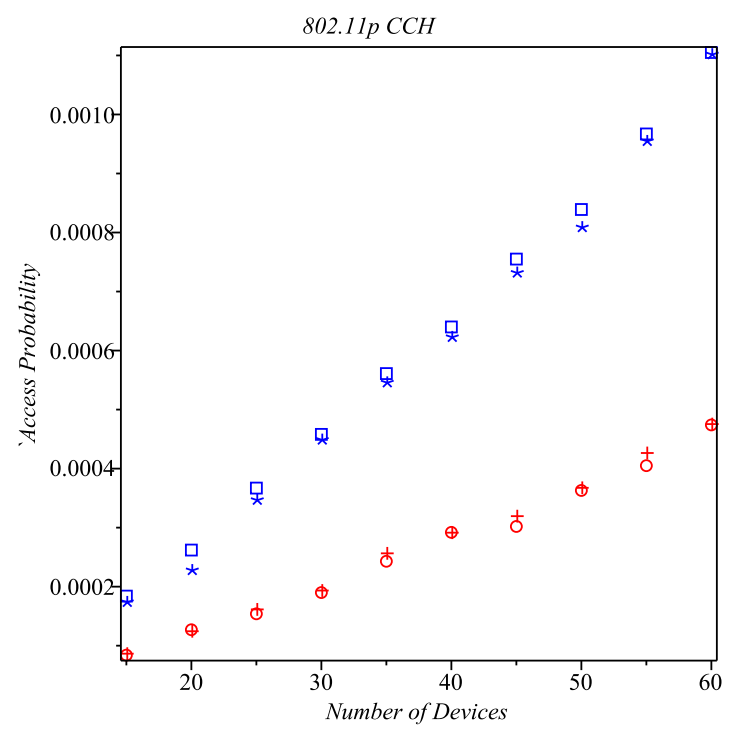

(a) Access Probability

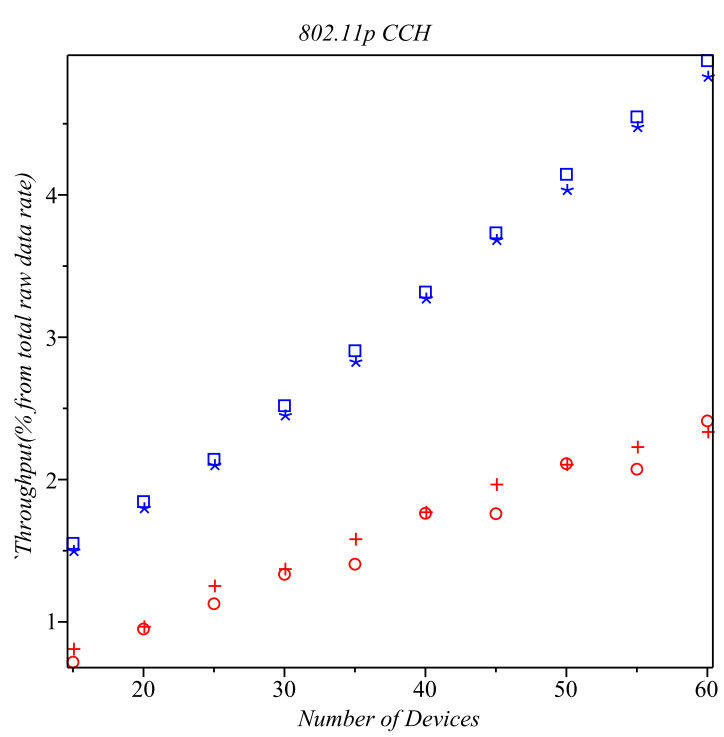

(b) Throughput

Figure 4.10: Access Probability and Throughput for 802.11p CCH for Duty Cycle equal to 0.6. 


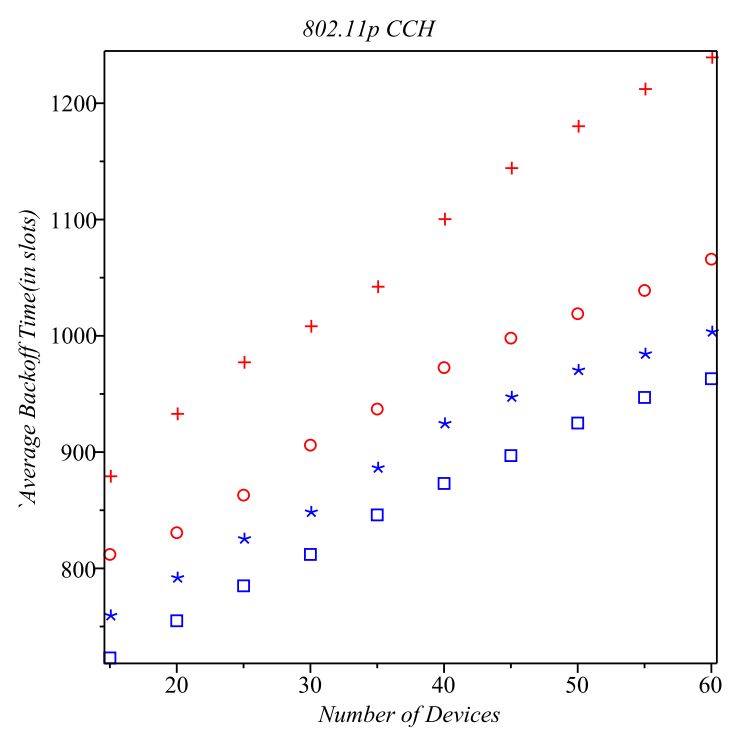

(a) Average Backoff Time

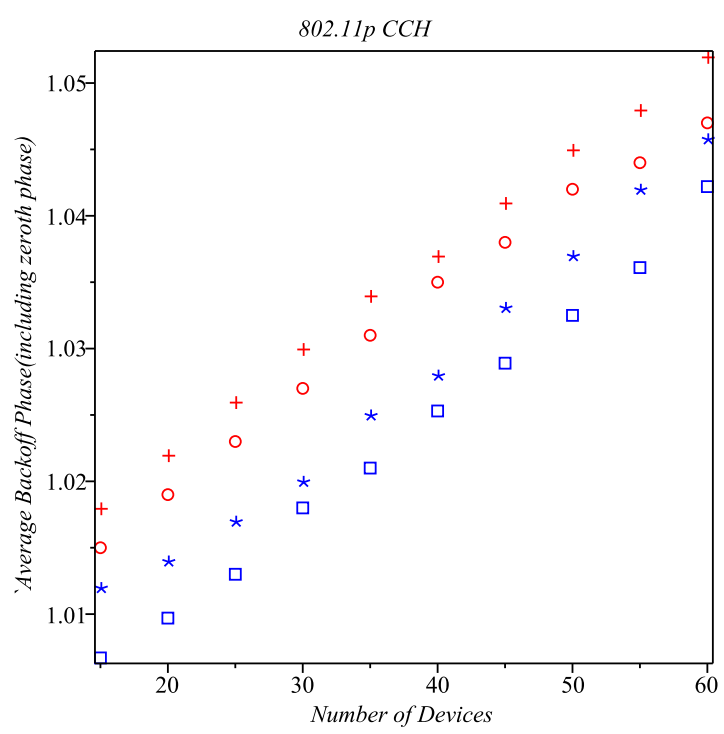

(b) Average Backoff Phase

Figure 4.11: Average Backoff Time and Average Backoff Phase for 802.11p CCH for Duty Cycle equal to 0.6 .

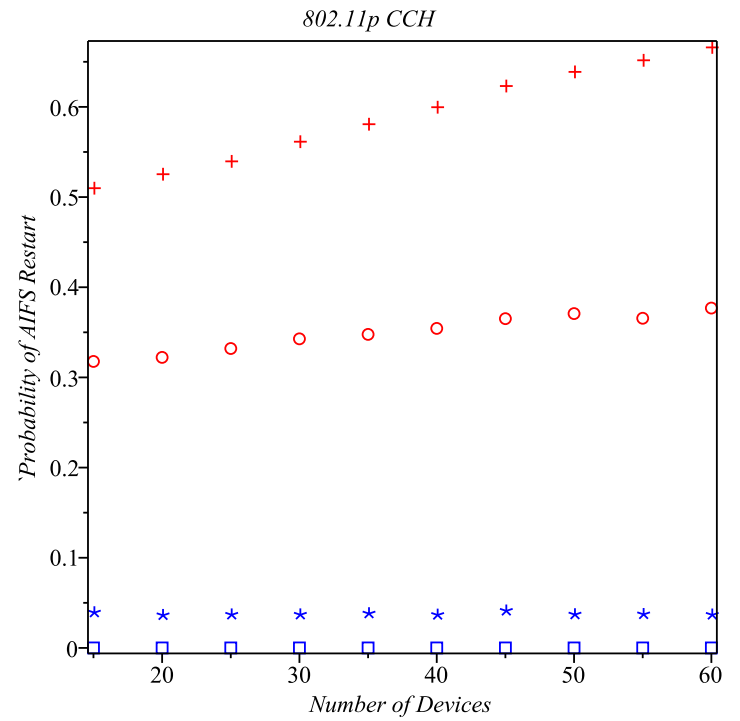

(a) AIFSN Restart

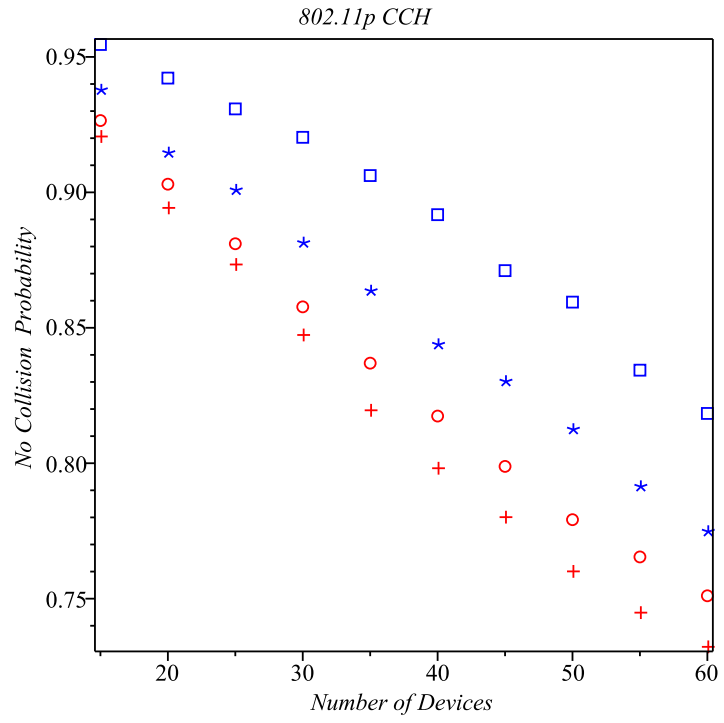

(b) No Collision Probability

Figure 4.12: AIFSN Restart Probability and No Collision Probability for 802.11p CCH for Duty Cycle equal to 0.6 .

DUTY CYCLE EQUAL TO 0.6 CCH: The throughput and access probability obtained is of 
linear trend which is expected as the number of devices increases. As VO and VI are the highest priority ACs they are expected to have the best throughput and access probability as we have obtained. The higher data rate plays a further role to minimize the effect of lower priority ACs. The average backoff time is highest for the $\mathrm{BK}$ as it is the lowest priority $\mathrm{AC}$ and it is interrupted maximum number of times during its countdown both in aifs and random count. This is proved by the fact when we see the aifsn restart probability value for these ACs. The average backoff phase value is increasing with $\mathrm{BK}$ having the highest value. $\mathrm{VO}$ would collide the least as it is expected to transmit easily because of the EDCA parameters giving it high priority along with higher data rate with $\mathrm{BK}$ being the worst performer as it has the lowest priority and also has a low data rate as compared to higher AC.

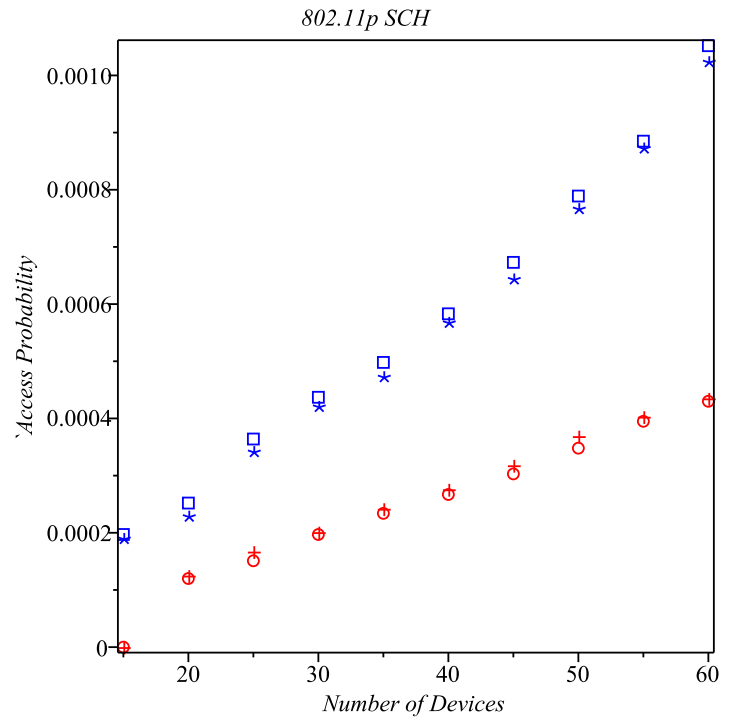

(a) Access Probability

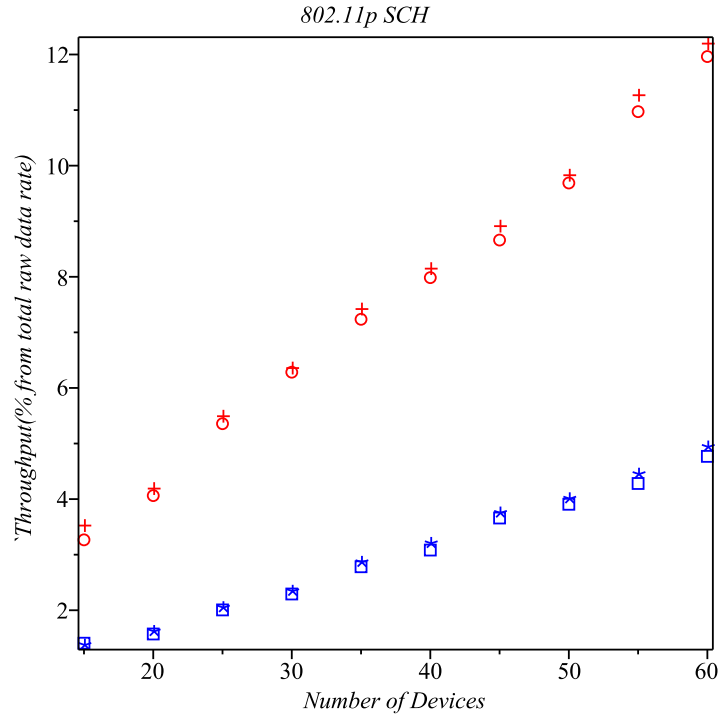

(b) Throughput

Figure 4.13: Access Probability and Throughput for $802.11 \mathrm{p} \mathrm{SCH}$ for Duty Cycle equal to 0.6. 


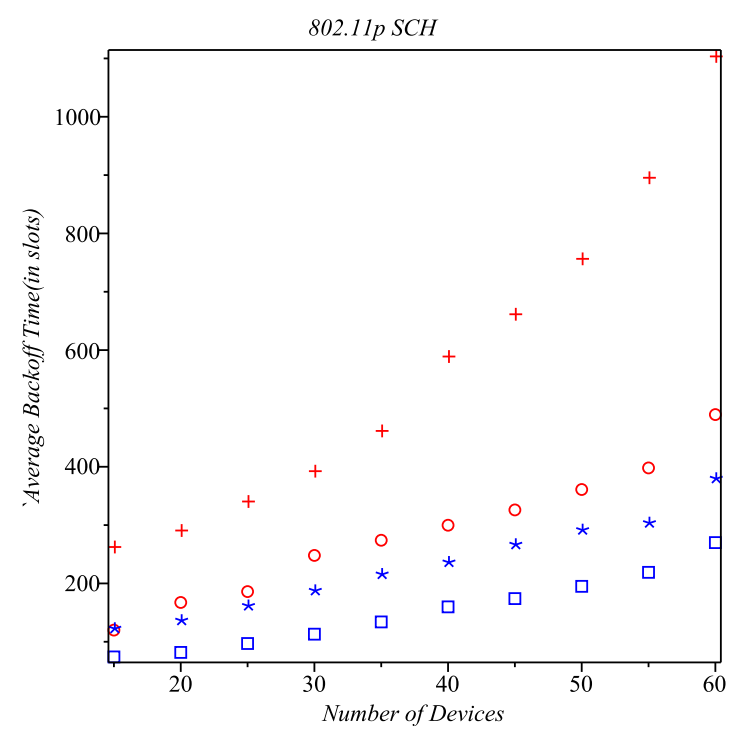

(a) Average Backoff Time

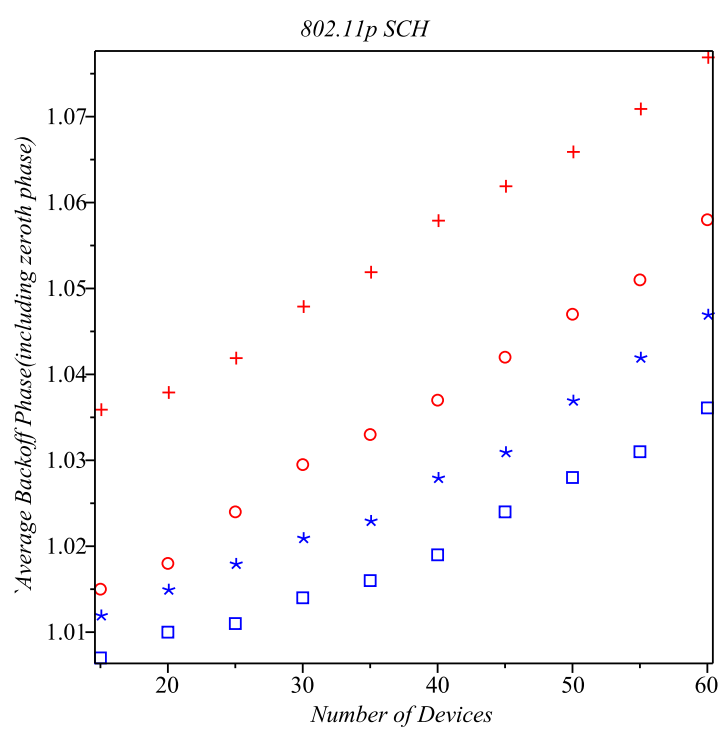

(b) Average Backoff Phase

Figure 4.14: Average Backoff Time and Average Backoff Phase for 802.11p SCH for Duty Cycle equal to 0.6 .

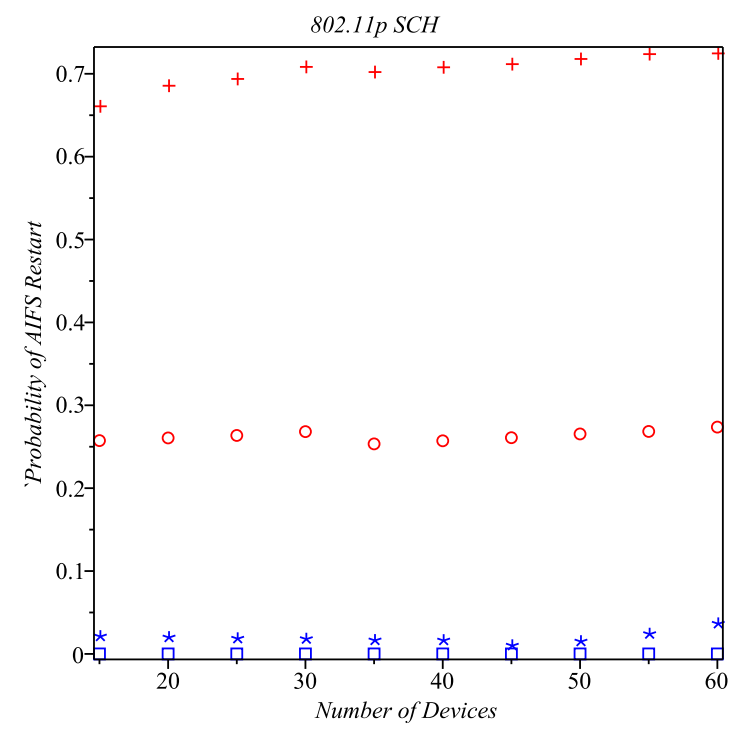

(a) AIFSN Restart

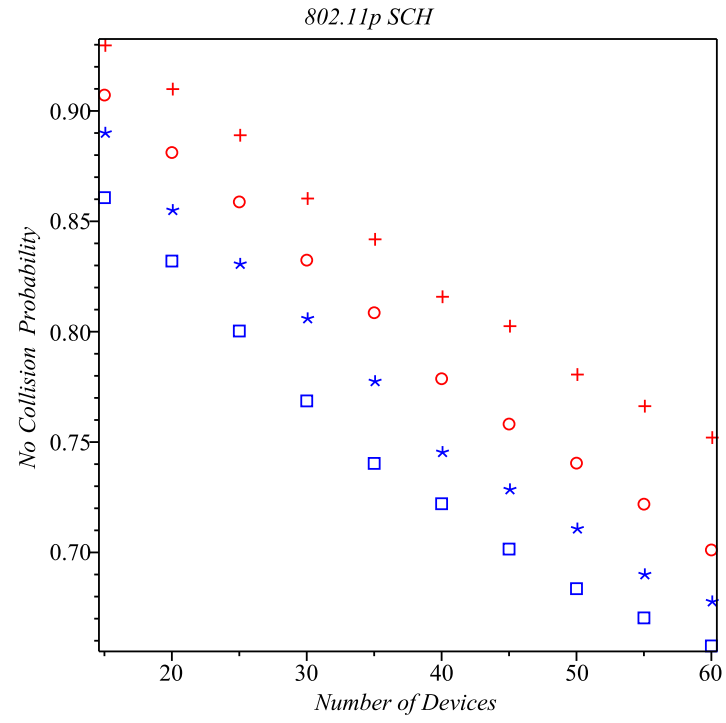

(b) No Collision Probability

Figure 4.15: AIFSN Restart Probability and No Collision Probability for $802.11 \mathrm{p} \mathrm{SCH}$ for Duty Cycle equal to 0.6 .

DUTY CYCLE EQUAL TO 0.6 SCH: The throughput and access probability obtained are 
linear in nature. Due to the high data rate assigned to the BK and BE and milder EDCA parameters they have higher values than VO and VI. The average backoff value is higher for BK as compared to others because of the higher AIFS value and bigger $\mathrm{CW}$ range which also affects the average backoff phase and the behaviour obtained. The aifsn restart value is highest for BK as expected whereas VO never restarts due to the synchronization with the previous transmission as it has the shortest AIFS value and no other AC can interrupt it. The no collision probability is the best for $\mathrm{BK}$ as the high data rate allows it to transmit without too many collision and VO collides maximum in this channel.

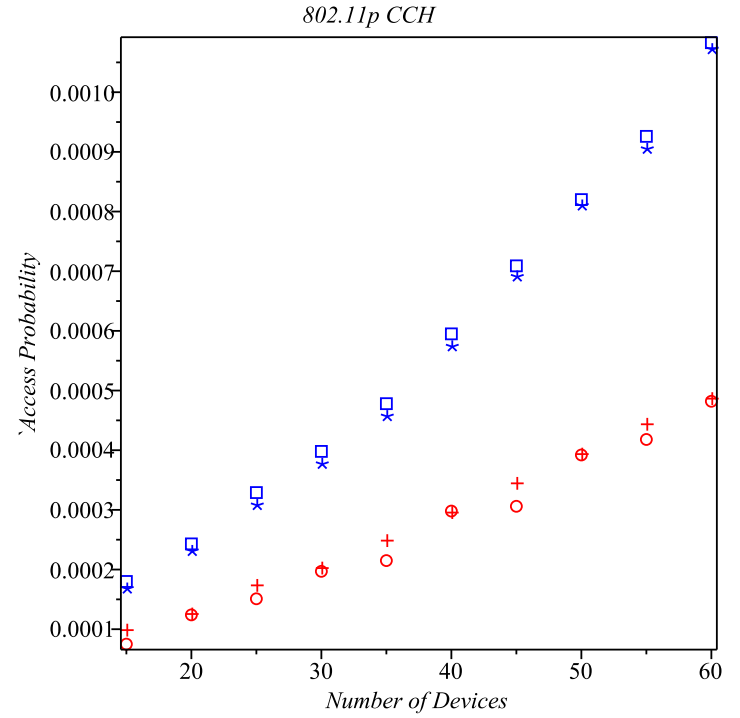

(a) Access Probability

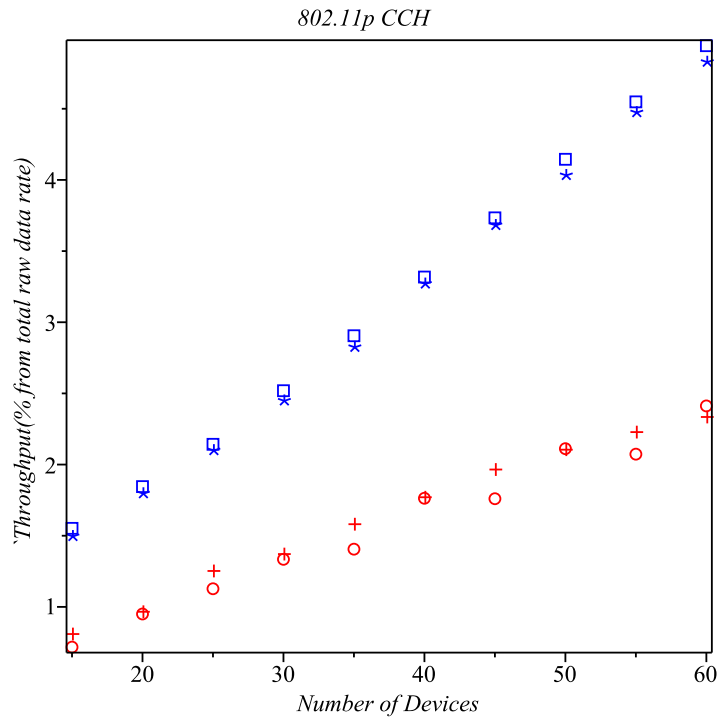

(b) Throughput

Figure 4.16: Access Probability and Throughput for 802.11p CCH for Duty Cycle equal to 0.5. 


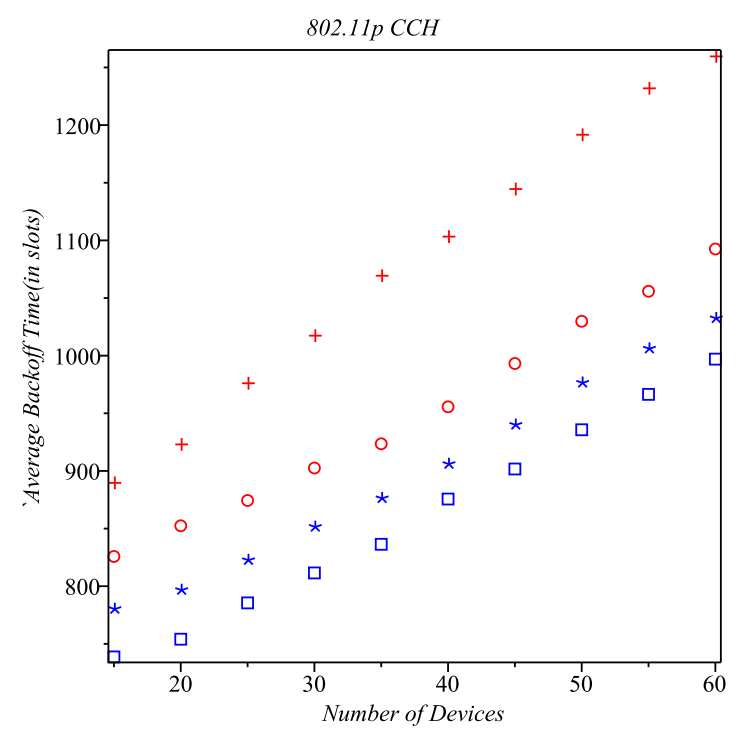

(a) Average Backoff Time

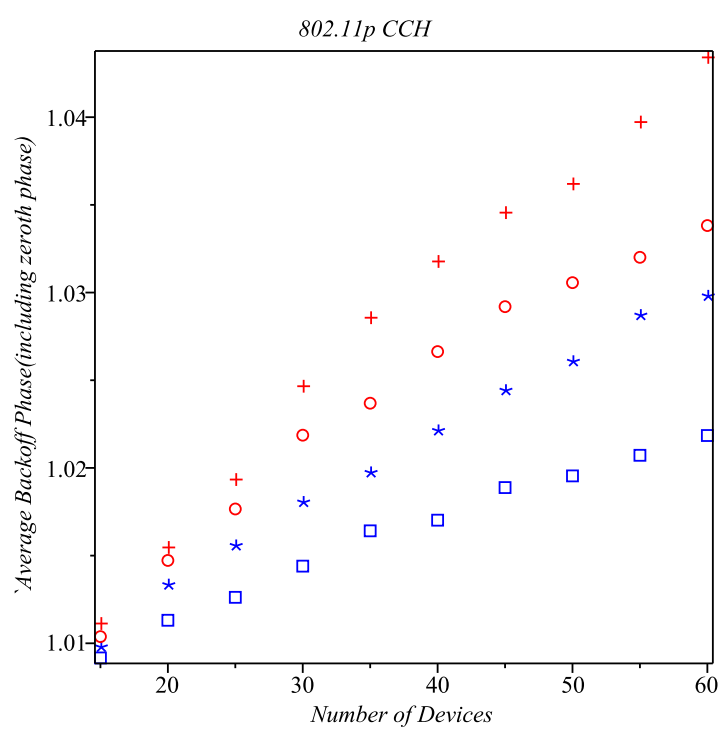

(b) Average Backoff Phase

Figure 4.17: Average Backoff Time and Average Backoff Phase for $802.11 \mathrm{p} \mathrm{CCH}$ for Duty Cycle equal to 0.5 .

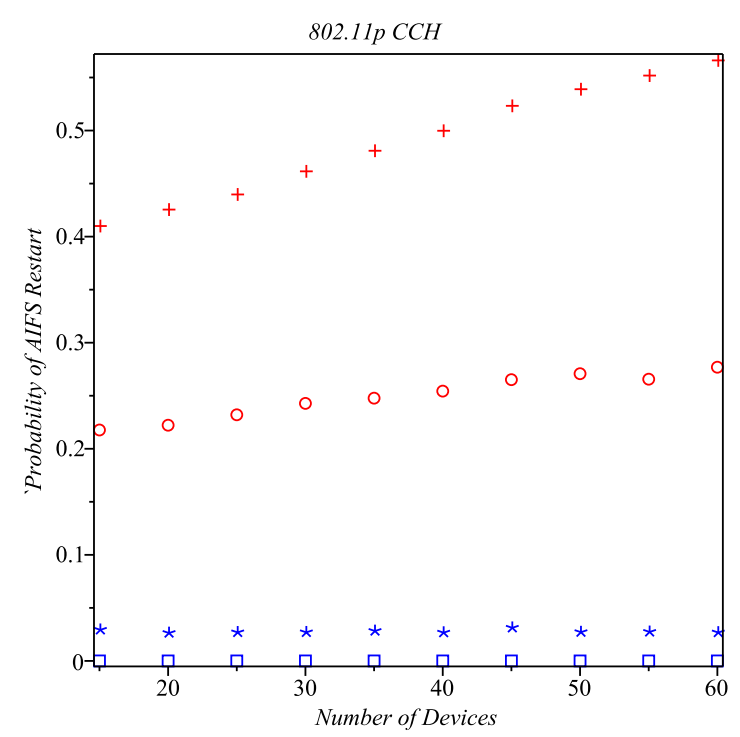

(a) AIFSN Restart

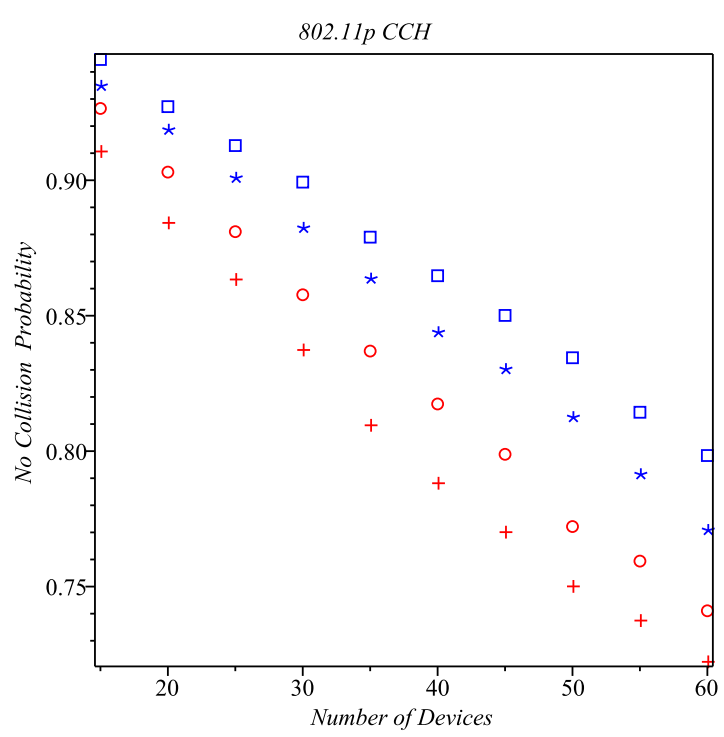

(b) No Collision Probability

Figure 4.18: AIFSN Restart Probability and No Collision Probability for 802.11p CCH for Duty Cycle equal to 0.5 .

DUTY CYCLE EQUAL TO $0.5 \mathrm{CCH}$ : The graphs above shows the performance parameters 
for the network at duty cycle 0.5 . In $\mathrm{CCH}$ duty cycle 0.5 the pattern for access probability and throughput are the same as obtained for 0.6. As on $\mathrm{CCH}$, voice(VO) and video(VI) should perform better because of shorter AIFSN and CW and higher data rate, the throughput and access probability is higher for them as compared to best effort(BE) and background(BK). The difference between the $\mathrm{BE}$ and $\mathrm{BK}$ throughput in the odd number of devices is due to the population of the nodes. When there are odd number of devices an extra device is assigned to BK causing a little difference in the throughput between $\mathrm{BE}$ and $\mathrm{BK}$ as we have more number of devices working for BK. The average backoff value would be higher than the one obtained in duty cycle $0.6 \mathrm{CCH}$ because in 0.6 it had the maximum bandwidth to complete the backoff however here the bandwidth has reduced and the transmissions which could not be completed in this active channel have to wait for the other channel i.e SCH to complete, so the waiting time increases which also increases the average backoff time. The average backoff phase value would show linear trend. The probability of aifsn getting restarted is maximum for the BK as it is most likely to be interrupted during its backoff because of its highest AIFSN value and high CW followed by BE and VI. VO would never be restarted because of the shortest AIFSN value and no other AC can interrupt it. The no collision probability decreases as compared to the $0.6 \mathrm{CCH}$ duty cycle because during the inactive period i.e SCH activity the packets would arrive in the buffer and thus when the channel becomes active more collisions occur. The no collision probability shows the trend that VO would be colliding the least as it has shortest AIFS value and CW and high data rate, so it would be transmitted most easily as compared to other type of data followed by VI, BE and BK would be the worst performer because of the parameters assigned. 


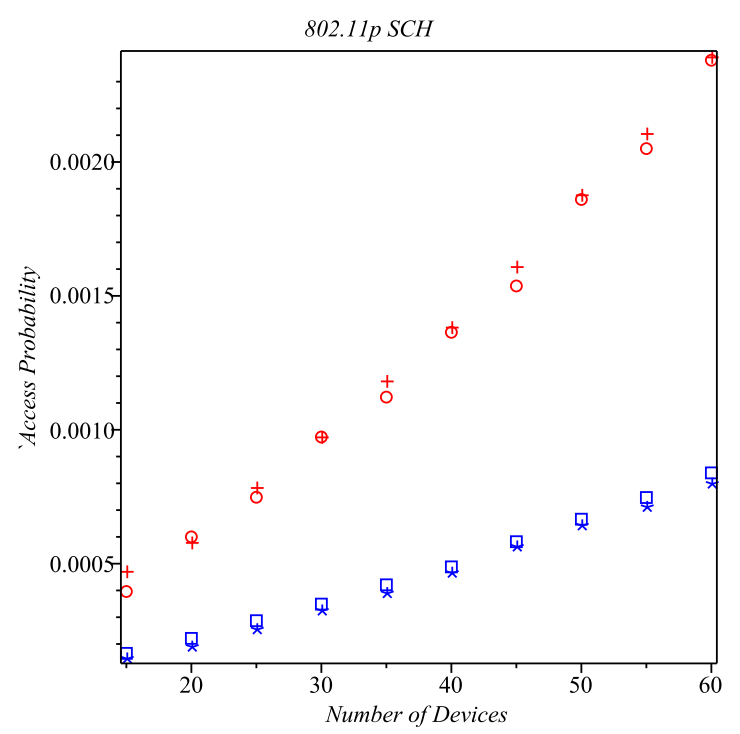

(a) Access Probability

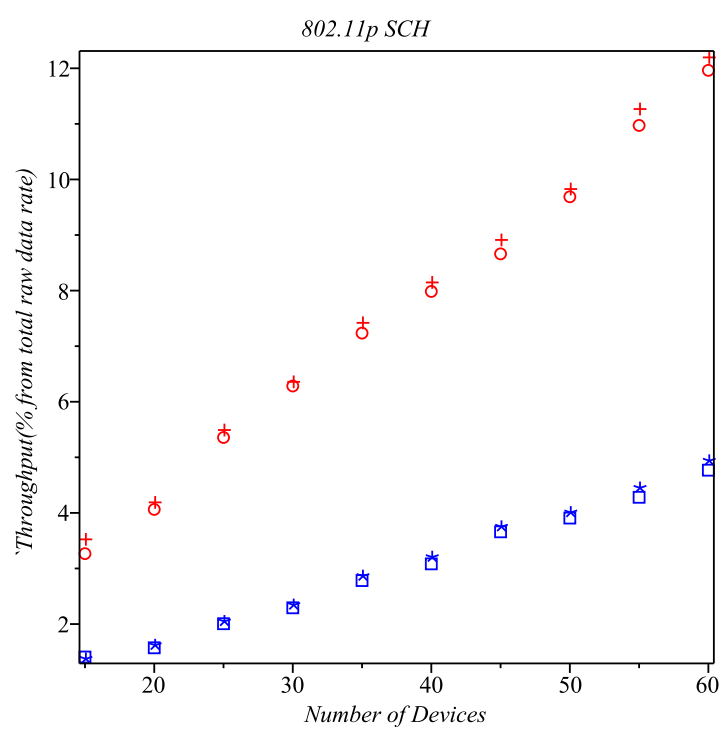

(b) Throughput

Figure 4.19: Access Probability and Throughput for $802.11 \mathrm{p} \mathrm{SCH}$ for Duty Cycle equal to 0.5 .

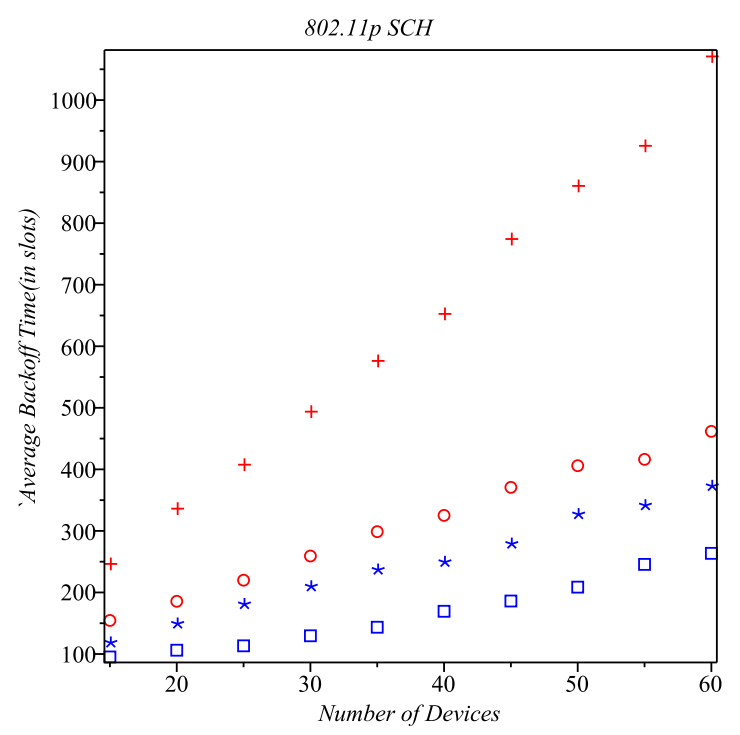

(a) Average Backoff Time

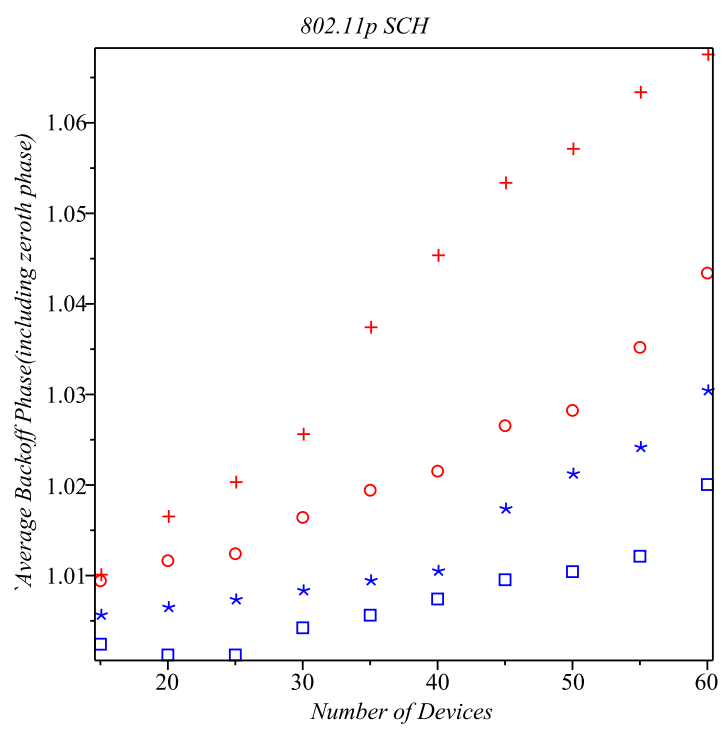

(b) Average Backoff Phase

Figure 4.20: Average Backoff Time and Average Backoff Phase for 802.11p SCH for Duty Cycle equal to 0.5 . 


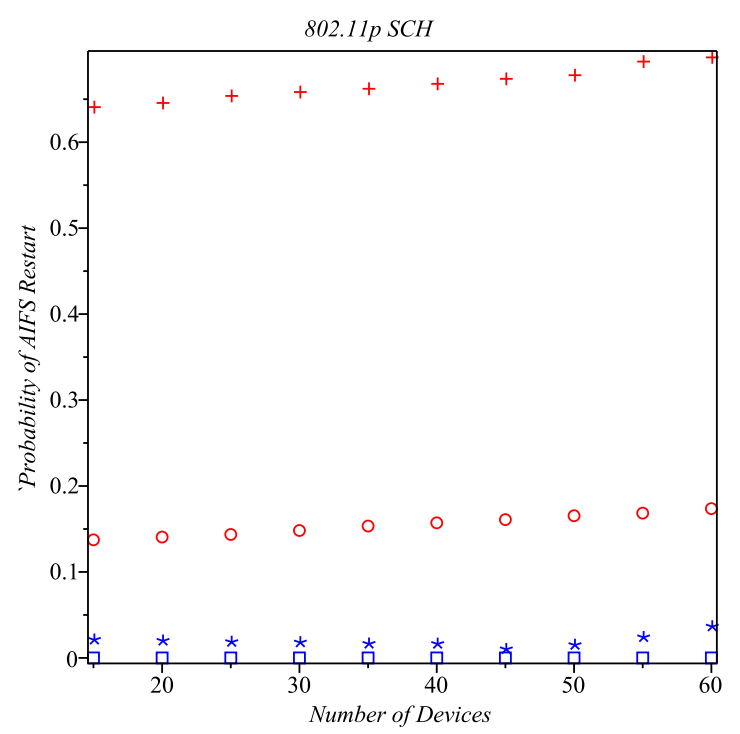

(a) AIFSN Restart

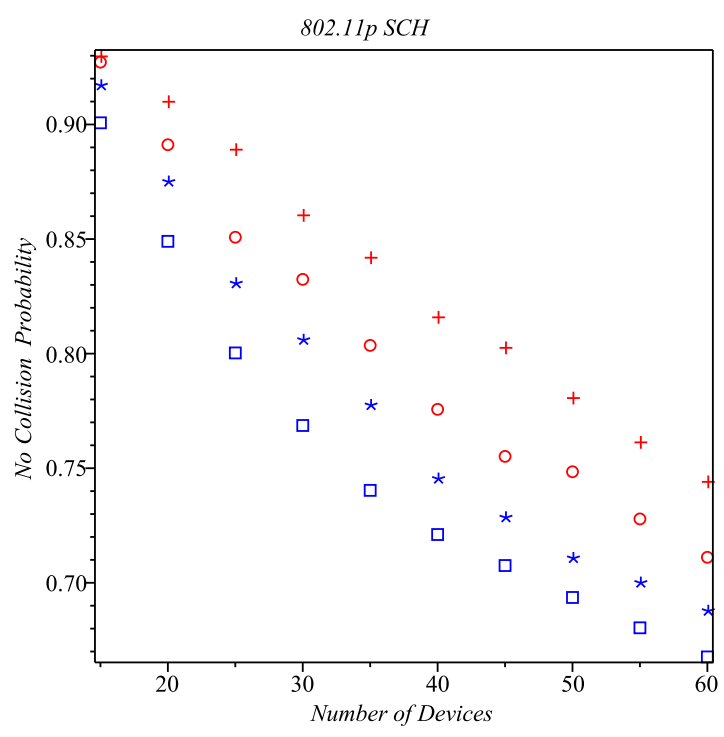

(b) No Collision Probability

Figure 4.21: AIFSN Restart Probability and No Collision Probability for 802.11p SCH for Duty Cycle equal to 0.5 .

DUTY CYCLE EQUAL TO 0.5 SCH: The graph above shows the trend of access probability and throughput increasing linearly. The important thing to notice here is that the BK would be performing the best here because of the high data rate and greater node population which diminishing the effect of milder EDCA parameters as compared to the VO and VI. The throughput is the highest for the $\mathrm{BK}$ and $\mathrm{BE}$ as compared to $\mathrm{VI}$ and VO. As the $\mathrm{CW}$ for $\mathrm{BK}$ and $\mathrm{BE}$ are similar they show similar behaviour and outperform the VI and VO. The average backoff time is highest for the BK as expected along with the average backoff phase. The probability of aifs restart is the highest for the BK as it would be interrupted the most. The no collision probability shows the BK would be performing best with lowest collisions on $\mathrm{SCH}$ and the high data rate would reduce the effect of AIFS and CW on its performance. The no collision probability would be increasing with the increase in bandwidth as the bandwidth for inactive channel would be less and less packets would arrive during the inactive period causing less collisions when the channel becomes active again for the next cycle. 


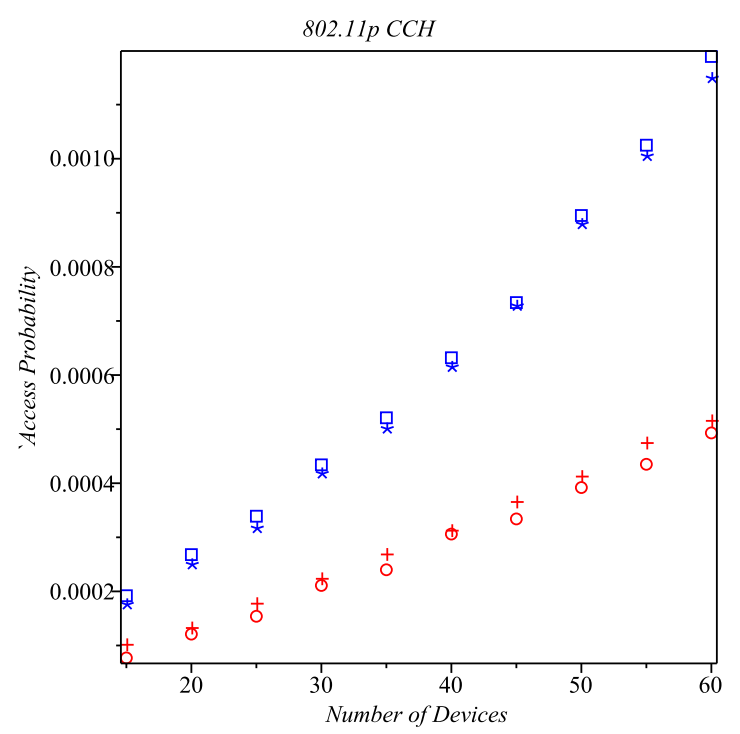

(a) Access Probability

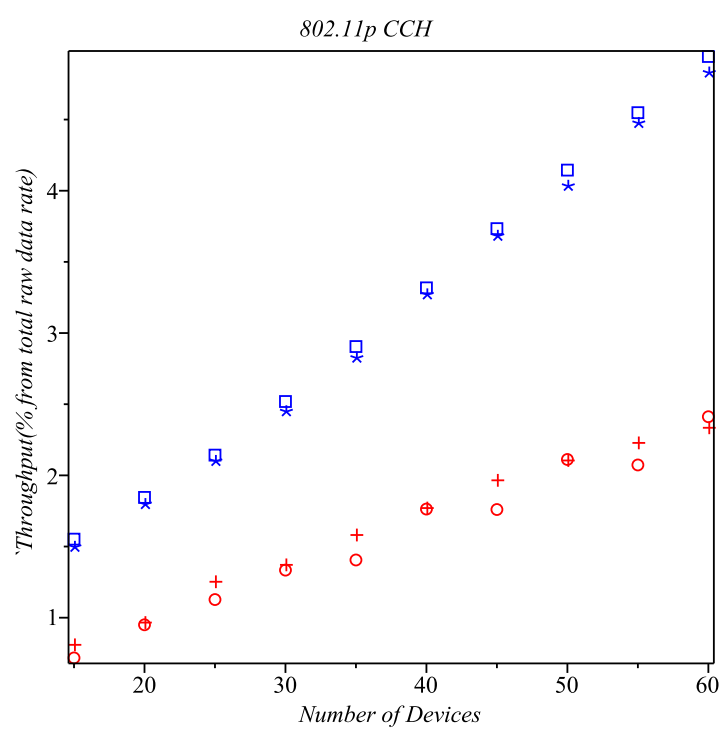

(b) Throughput

Figure 4.22: Access Probability and Throughput for $802.11 \mathrm{p} \mathrm{CCH}$ for Duty Cycle equal to 0.4 .

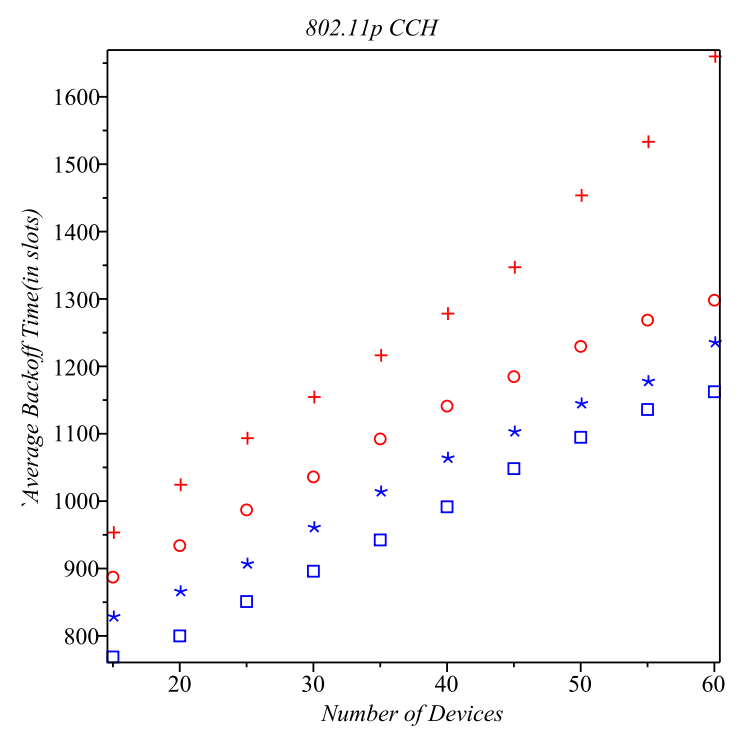

(a) Average Backoff Time

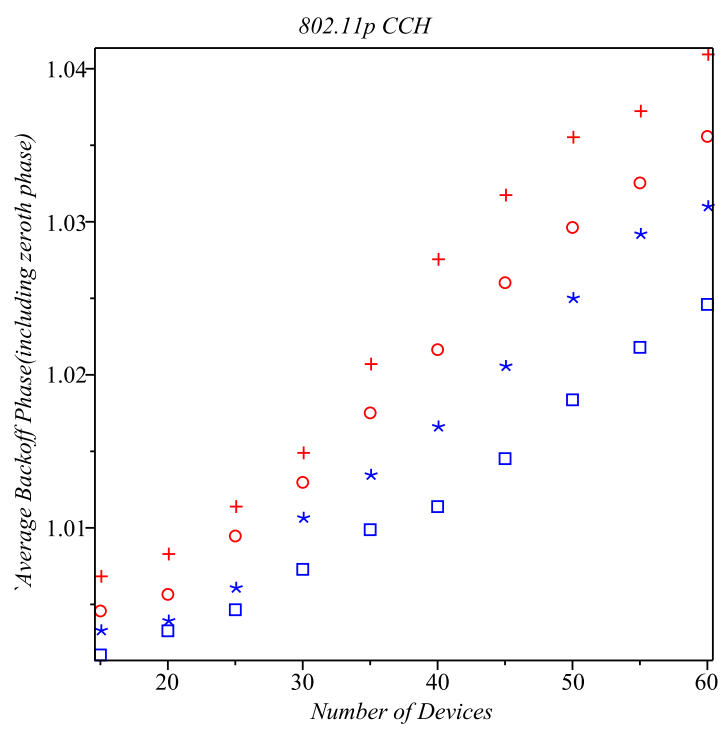

(b) Average Backoff Phase

Figure 4.23: Average Backoff Time and Average Backoff Phase for $802.11 \mathrm{p} \mathrm{CCH}$ for Duty Cycle equal to 0.4 . 


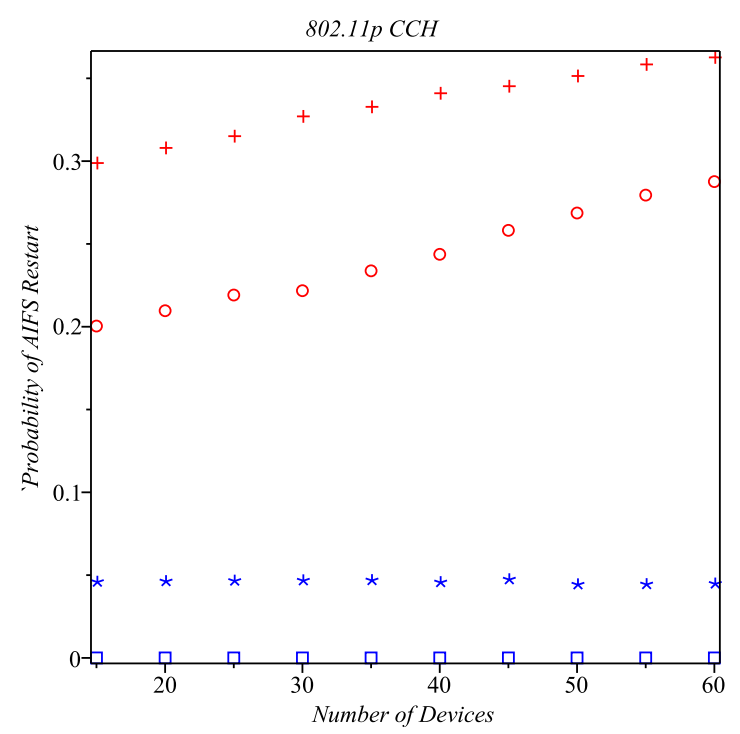

(a) AIFSN Restart

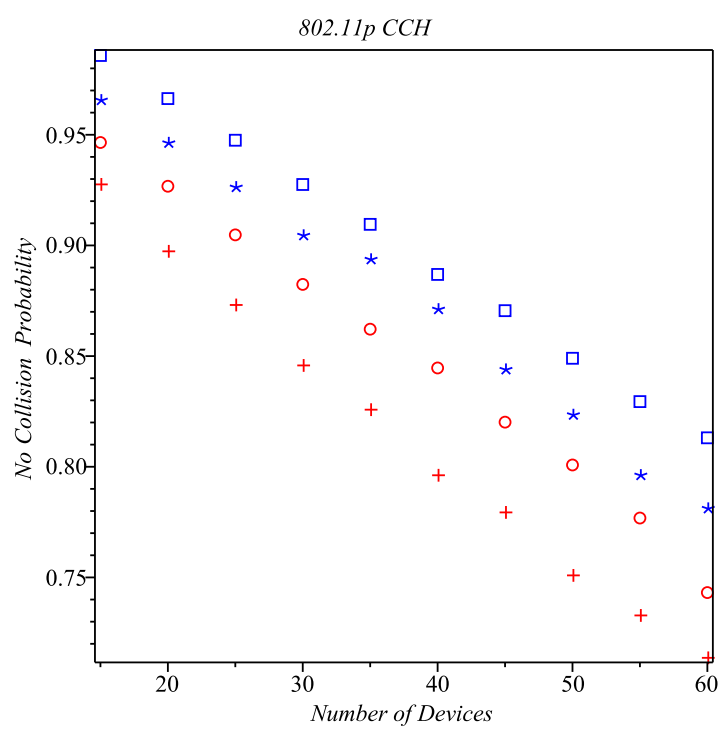

(b) No Collision Probability

Figure 4.24: AIFSN Restart Probability and No Collision Probability for 802.11p CCH for Duty Cycle equal to 0.4 .

DUTY CYCLE EQUAL TO 0.4 CCH: During this the access probability and throughput shows the same linear trend. The average backoff time increases as compared to the one obtained with the duty cycle of $0.5 \mathrm{CCH}$ as bandwidth of $\mathrm{CCH}$ decreases and $\mathrm{SCH}$ increases so the time taken to complete the backoff which could not complete in the same channel has to wait for the SCH period (which is inactive period for $\mathrm{CCH}$ ) to complete the backoff. The average backoff phase shows the linear trend and is highest for the BK with VO being the lowest. The AIFS value as compared to the duty cycle of 0.5 is less but still it shows the increasing trend with the increase in the number of devices. The no collision probability decreases further as compared to the duty cycle of 0.5 with the same reasoning for the one in 0.5 duty cycle of $\mathrm{CCH}$. 


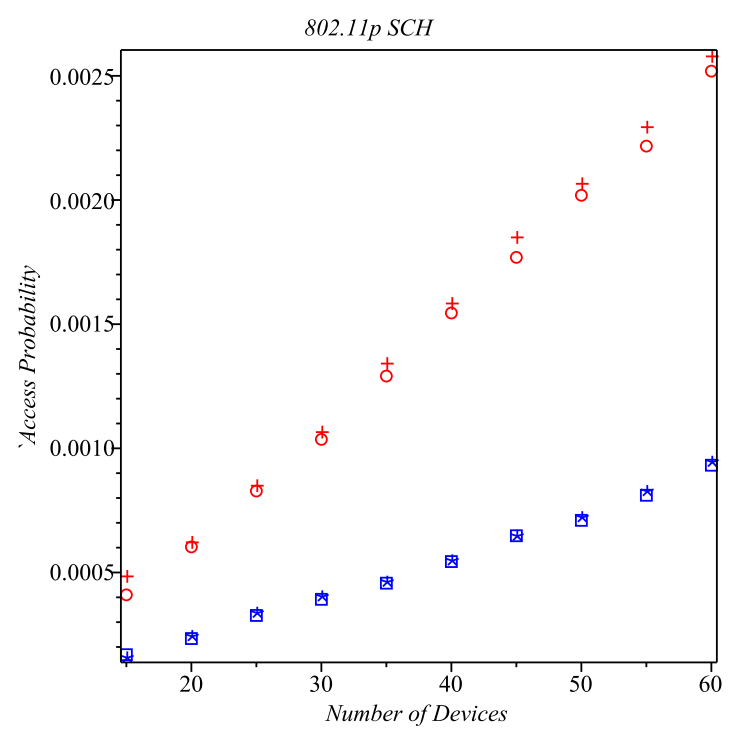

(a) Access Probability

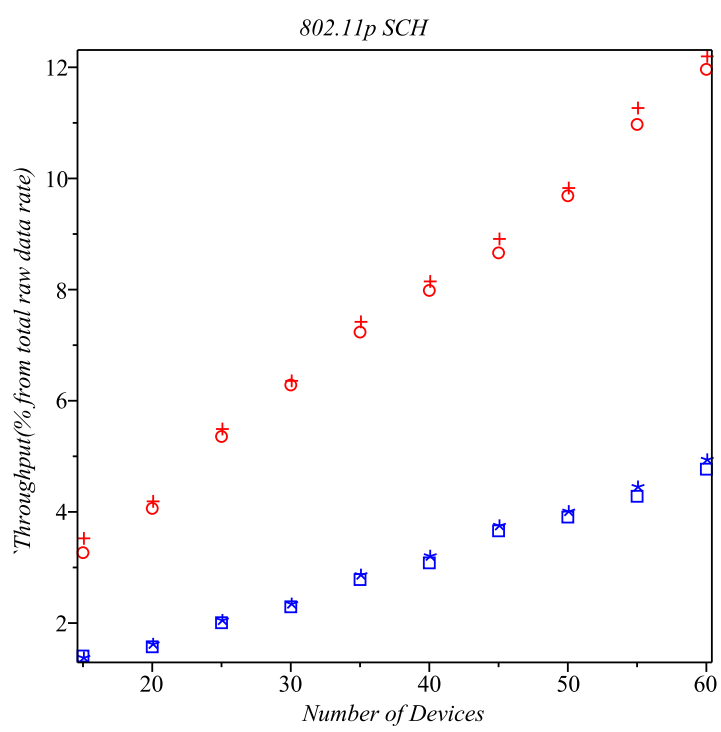

(b) Throughput

Figure 4.25: Access Probability and Throughput for $802.11 \mathrm{p} \mathrm{SCH}$ for Duty Cycle equal to 0.4.

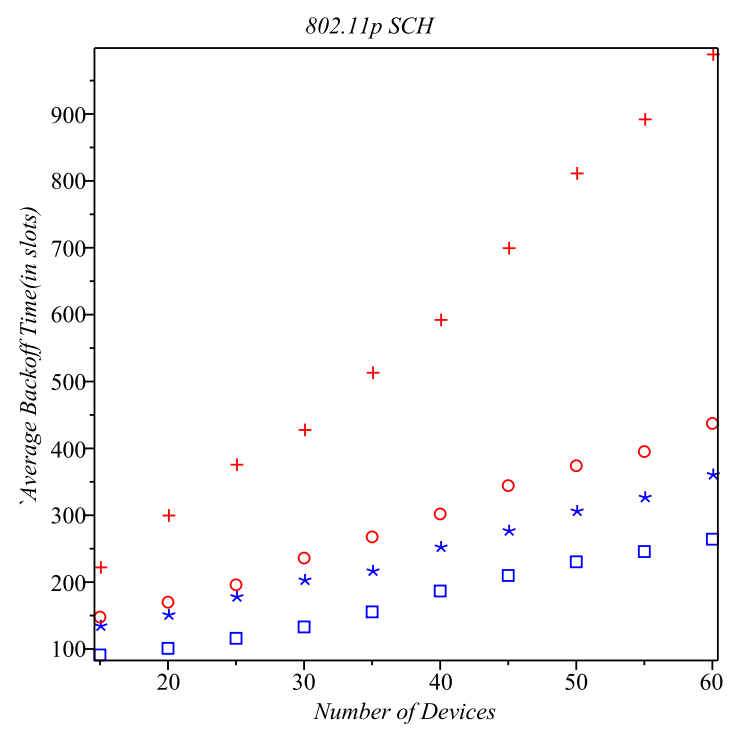

(a) Average Backoff Time

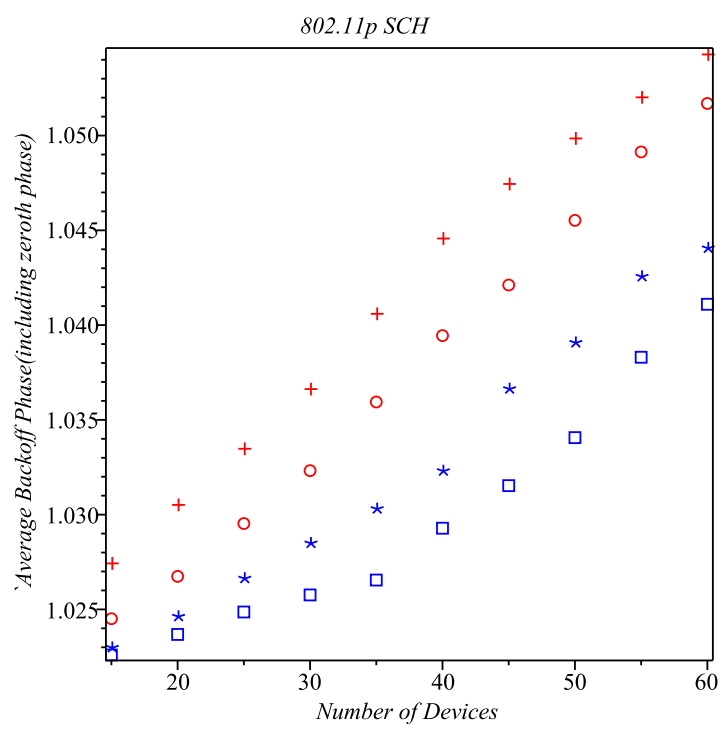

(b) Average Backoff Phase

Figure 4.26: Average Backoff Time and Average Backoff Phase for 802.11p SCH for Duty Cycle equal to 0.4 . 


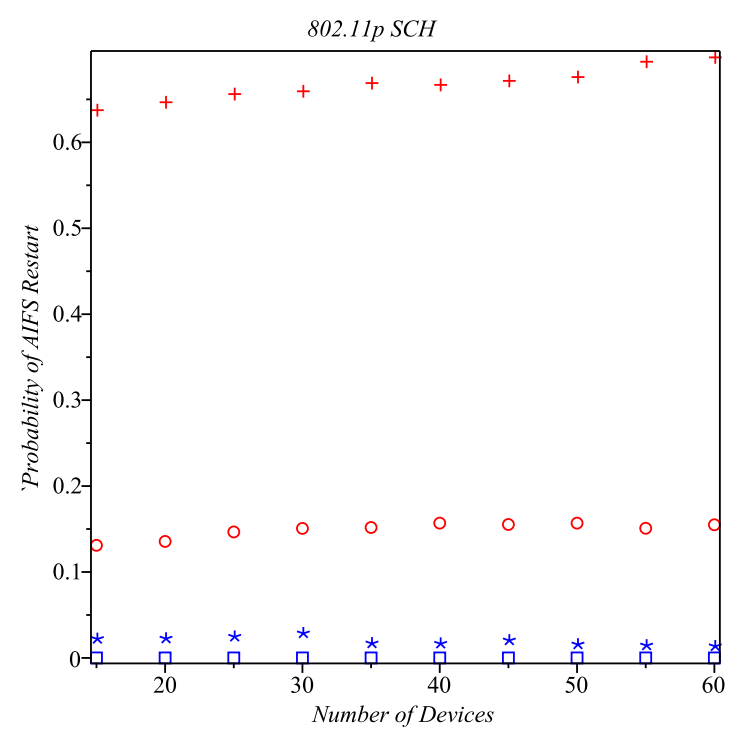

(a) AIFSN Restart

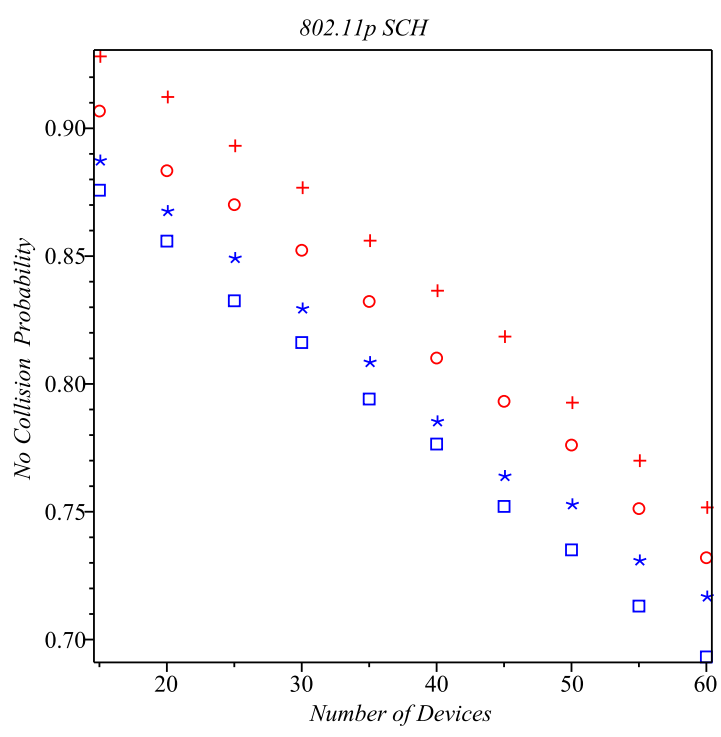

(b) No Collision Probability

Figure 4.27: AIFSN Restart Probability ann No Collision Probability for 802.11p SCH for Duty Cycle equal to 0.4 .

DUTY CYCLE EQUAL TO 0.4 SCH: The access probability and throughput shows the same trend as in the duty cycle of 0.5 for $\mathrm{SCH}$. The average backoff time shows the value which is lower than the value obtained in the duty cycle of 0.5 for $\mathrm{SCH}$. The average backoff value is decreasing as more bandwidth is available for the transmission to be completed and less waiting time(the other channel activity). The average backoff phase value shows the same linear trend. The AIFS restart value decreased slightly but it has the general trend of increasing with the increase in the number of devices. As compared to the duty cycle of 0.5 the no collision probability increases for all the different $\mathrm{AC}$ in this case due to greater bandwidth available for transmissions and the reason explained above in the duty cycle $0.5 \mathrm{SCH}$. 


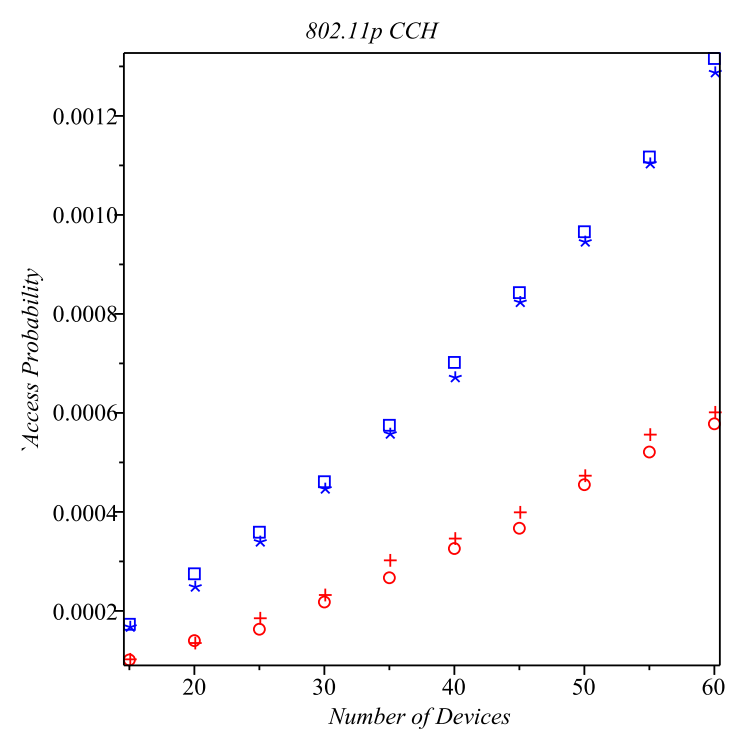

(a) Access Probability

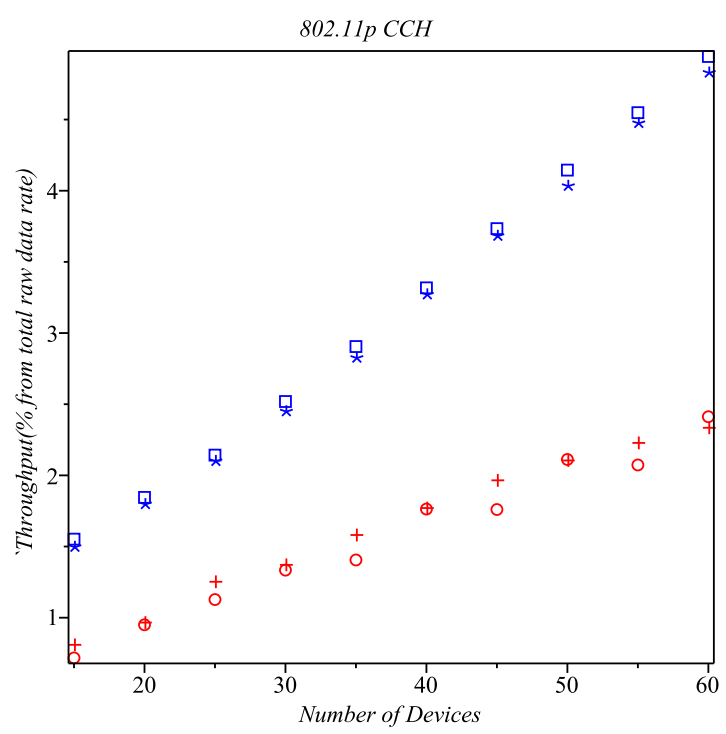

(b) Throughput

Figure 4.28: Access Probability and Throughput for $802.11 \mathrm{p} \mathrm{CCH}$ for Duty Cycle equal to 0.3.

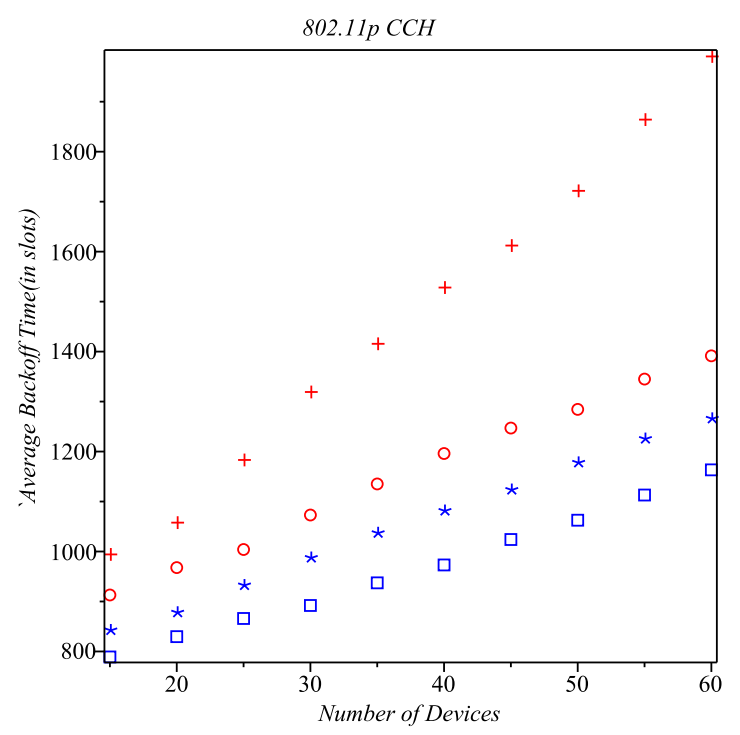

(a) Average Backoff Time

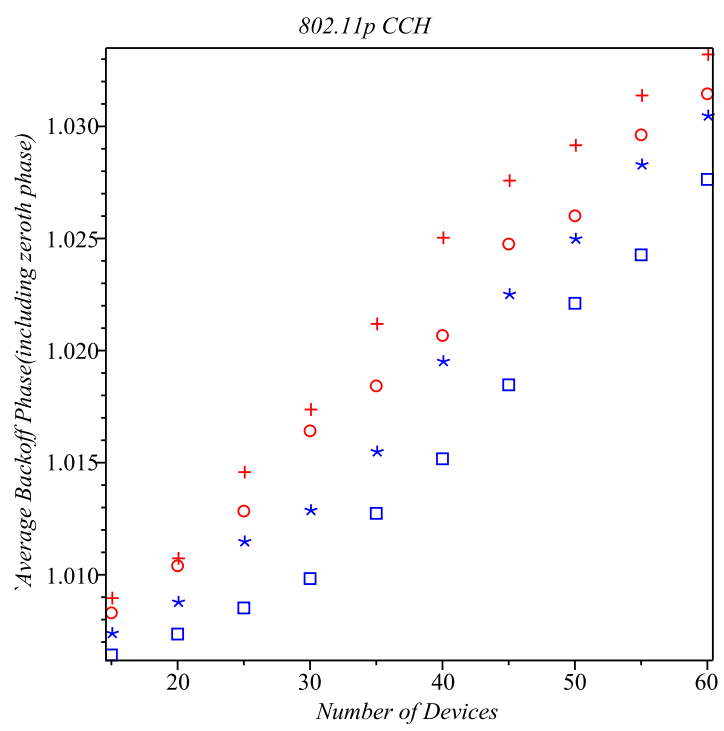

(b) Average Backoff Phase

Figure 4.29: Average Backoff Time and Average Backoff Phase for $802.11 \mathrm{p} \mathrm{CCH}$ for Duty Cycle equal to 0.3 . 


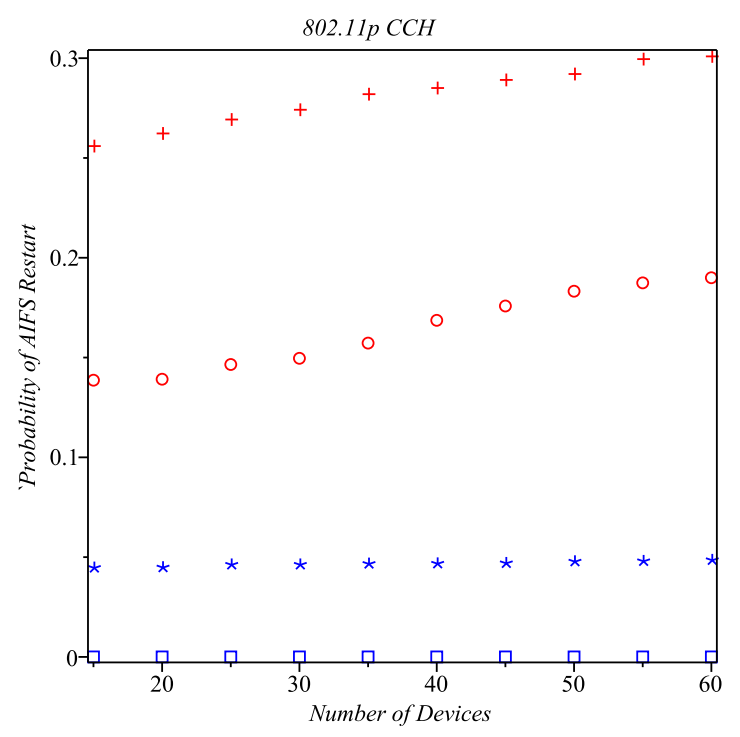

(a) AIFSN Restart

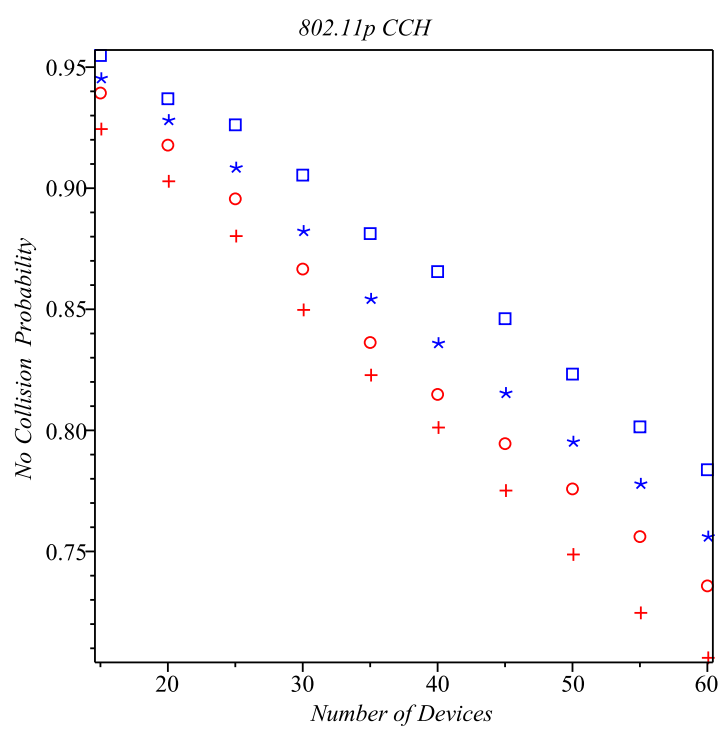

(b) No Collision Probability

Figure 4.30: AIFSN Restart Probability and No Collision Probability for 802.11p CCH for Duty Cycle equal to 0.3 .

DUTY CYCLE EQUAL TO 0.3 CCH: The access probability and throughput shows the same trend as observed in the previous duty cycles. The average backoff time increases further in the $\mathrm{CCH}$ for this duty cycle for the same reason as bandwidth reduces further for the $\mathrm{CCH}$ and increases for the SCH thereby increasing the average backoff time as for the incomplete transmission it has to wait for the $\mathrm{SCH}$ to complete. Due to this reason the average backoff phase value shows the same trend but its value is less as compared to the one obtained the other duty cycles. The AIFS restart value increases with the increase in the number of devices but as compared to the other duty cycles the value is lower. The no collision probability decreases further as compared to the other duty cycles as the bandwidth available is least in this case and once the SCH completes its cycle the packets that would have queued in the buffer would be transmitted causing most collisions. 


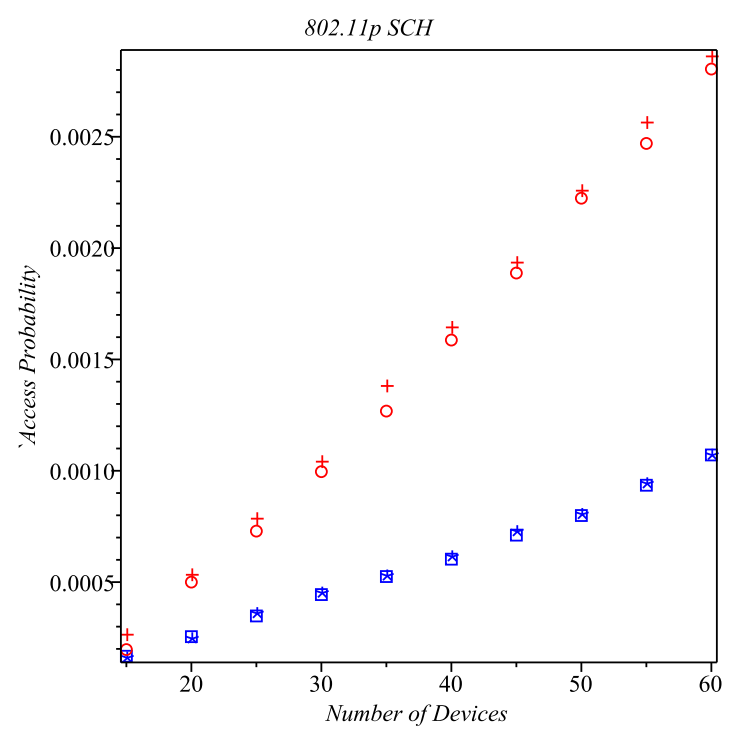

(a) Access Probability

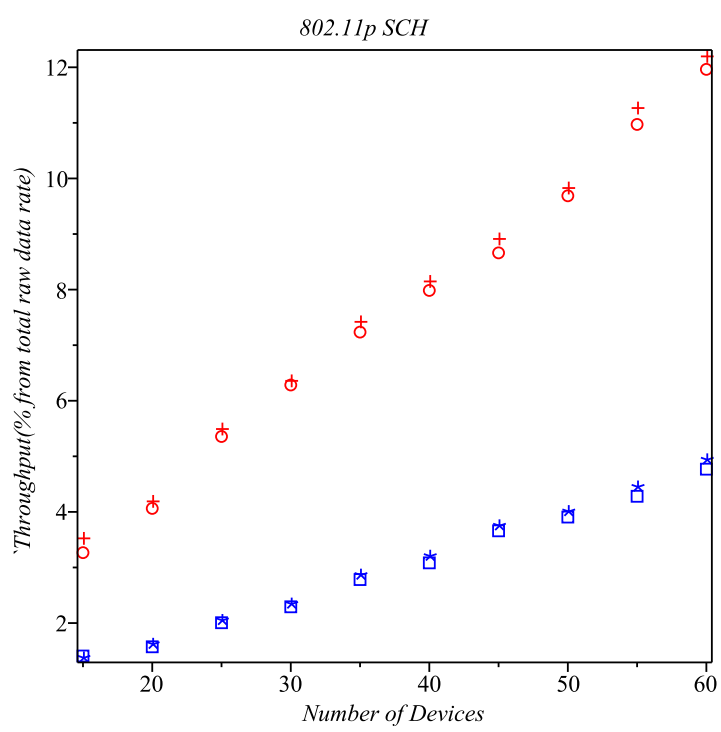

(b) Throughput

Figure 4.31: Access Probability and Throughput for $802.11 \mathrm{p} \mathrm{SCH}$ for Duty Cycle equal to 0.3 .

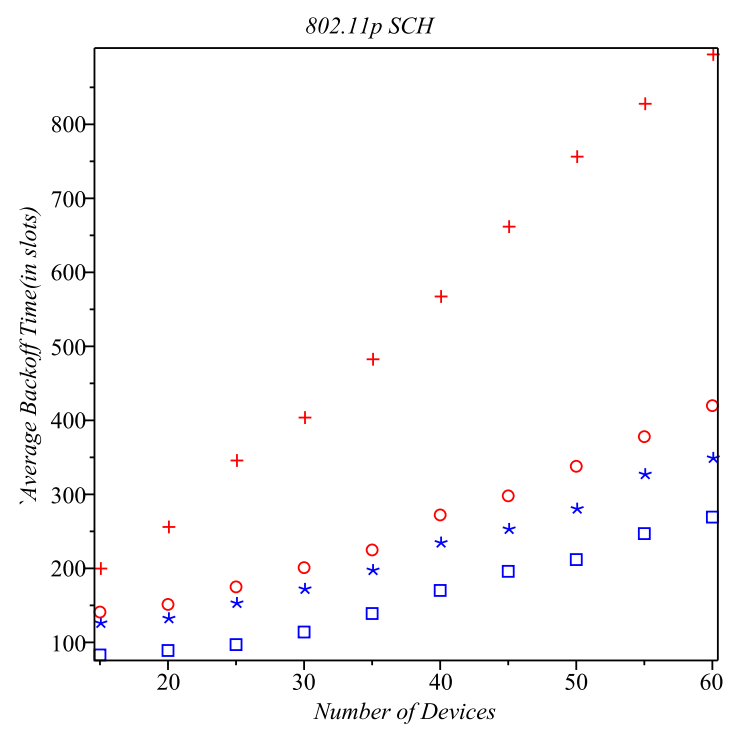

(a) Average Backoff Time

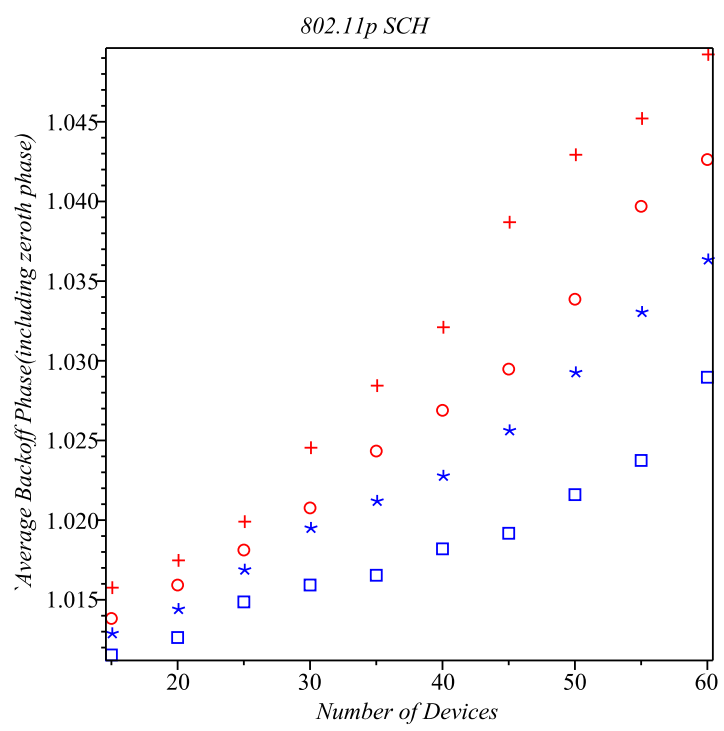

(b) Average Backoff Phase

Figure 4.32: Average Backoff Time and Average Backoff Phase for 802.11p SCH for Duty Cycle equal to 0.3 . 


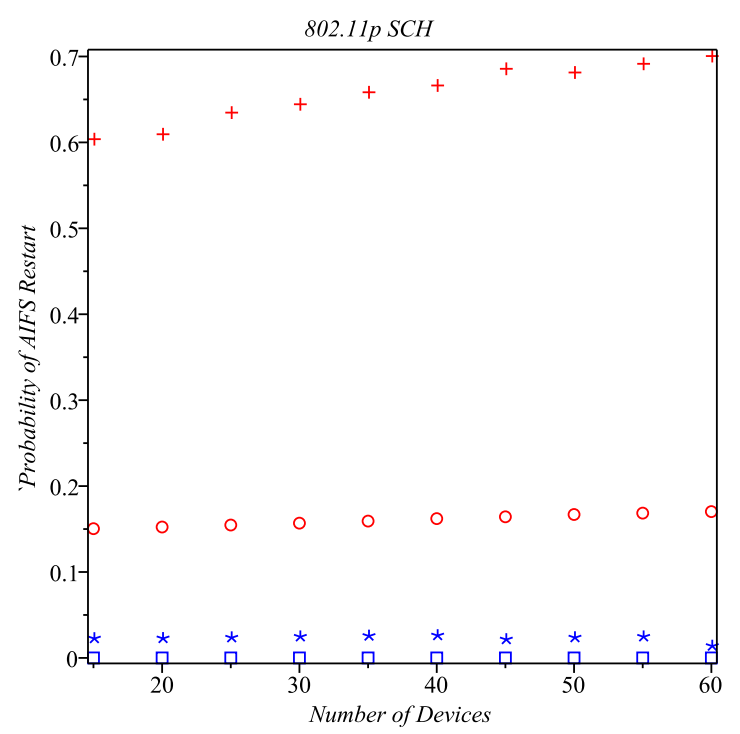

(a) AIFSN Restart

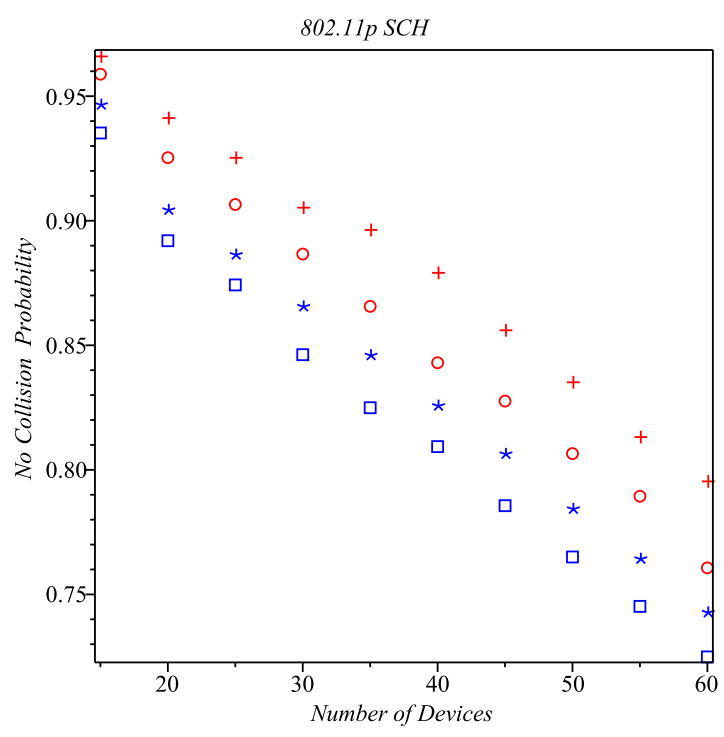

(b) No Collision Probability

Figure 4.33: AIFSN Restart Probability and No Collision Probability for 802.11p SCH for Duty Cycle equal to 0.3 .

DUTY CYCLE EQUAL TO 0.3 SCH: The access probability and throughput has the same trend. The average backoff value would decrease and would be lowest as compared to the other duty cycles with average backoff phase value showing the linear trend. The AIFS restart value is increasing with the number of devices. The no collision probability is increasing and is maximum as compared to other duty cycle for $\mathrm{SCH}$ as most transmissions would be completed in this channel and less packets would be accumulated when the other channel $\mathrm{CCH}$ is working and when it switch backs to the SCH less packets would collide.

\subsection{High Data Rate Experiment}

We performed another experiment by increasing the data rate for $\mathrm{BE}$ and $\mathrm{BK}$ on $\mathrm{SCH}$ and keeping all the other data rates the same as in previous experiments. The new data rates used in this experiment are mentioned in table 4.4.

Inorder to see the performance of these ACs with a higher data rate on $\mathrm{SCH}$ we did this exper- 
Table 4.4: New Data Rate Used

ACCESS CATEGORY SCH DATA RATE

\begin{tabular}{cl}
\hline Voice(VO) & $12 \mathrm{Kbps}$ \\
Video(VI) & $12 \mathrm{Kbps}$ \\
Best Effort(BE) & $40 \mathrm{Kbps}$ \\
Background(BK) & $40 \mathrm{Kbps}$
\end{tabular}

iment. We chose these higher data rates so as to see how the performance is affected when high data rate is used. Specifically our aim was to see if these ACs get into saturation and if they do how do they behave under these high load conditions. As we have not changed the data rates for $\mathrm{CCH}$ it won't be affected by this change in the $\mathrm{SCH}$ and so we would just analyse the performance of the $\mathrm{SCH}$ in this experiment.

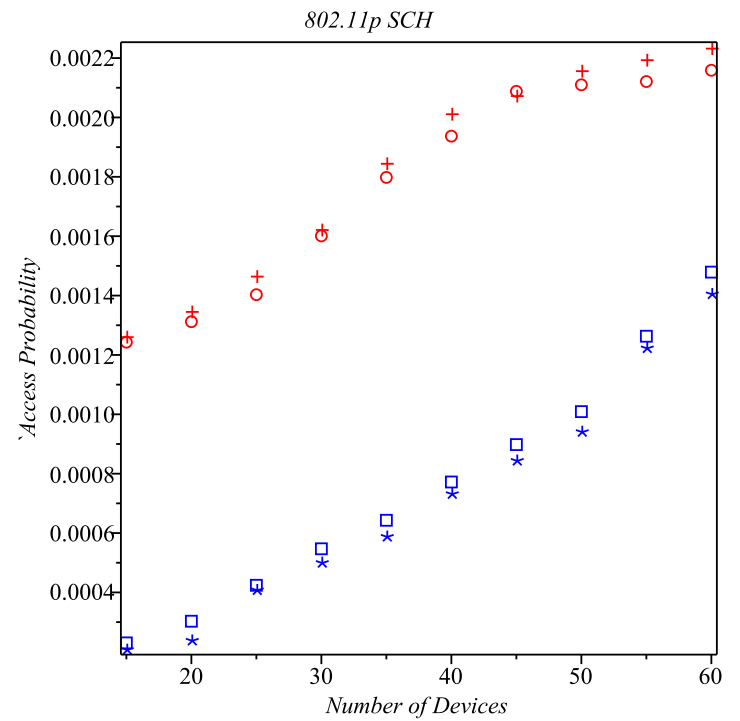

(a) Access Probability

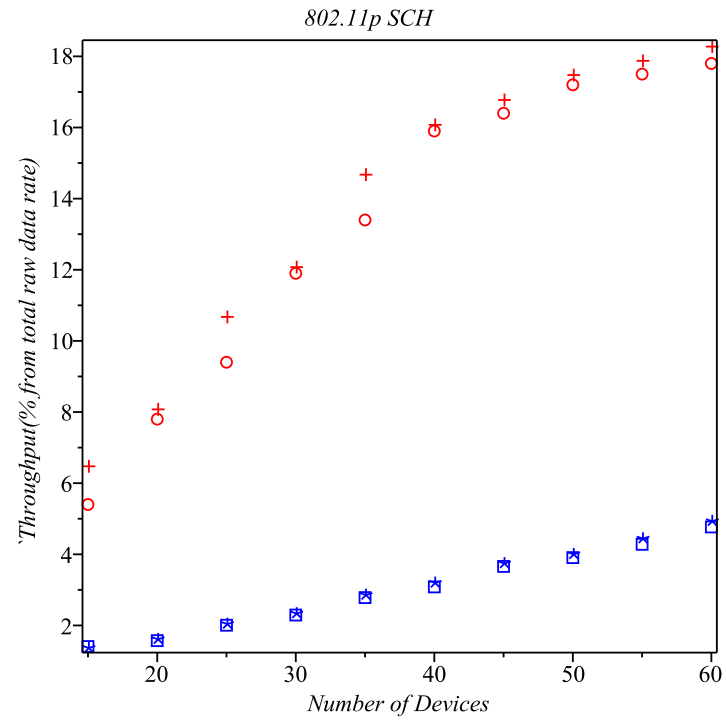

(b) Throughput

Figure 4.34: Access Probability and Throughput for 802.11p SCH for Duty Cycle equal to 0.6. 


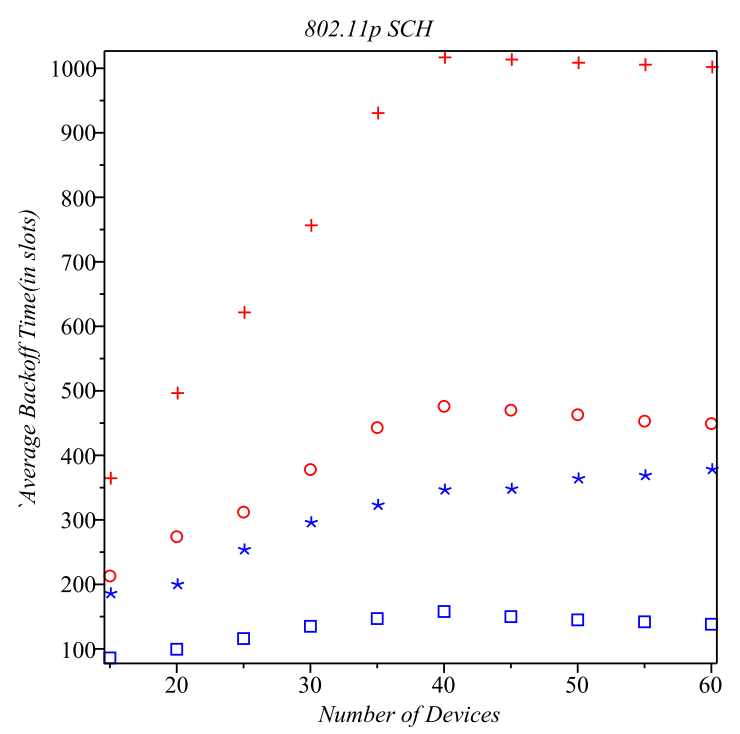

(a) Average Backoff Time

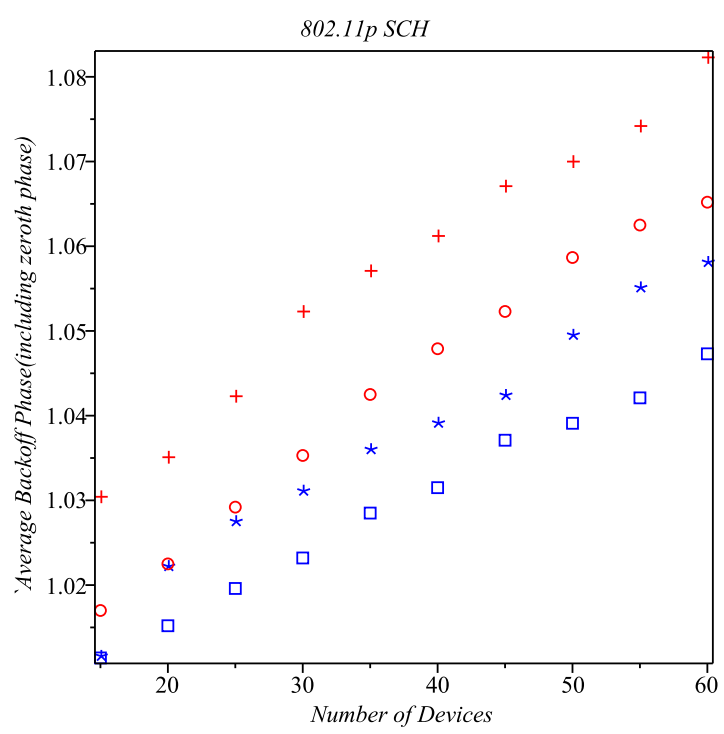

(b) Average Backoff Phase

Figure 4.35: Average Backoff Time and Average Backoff Phase for 802.11p SCH for Duty Cycle equal to 0.6 .

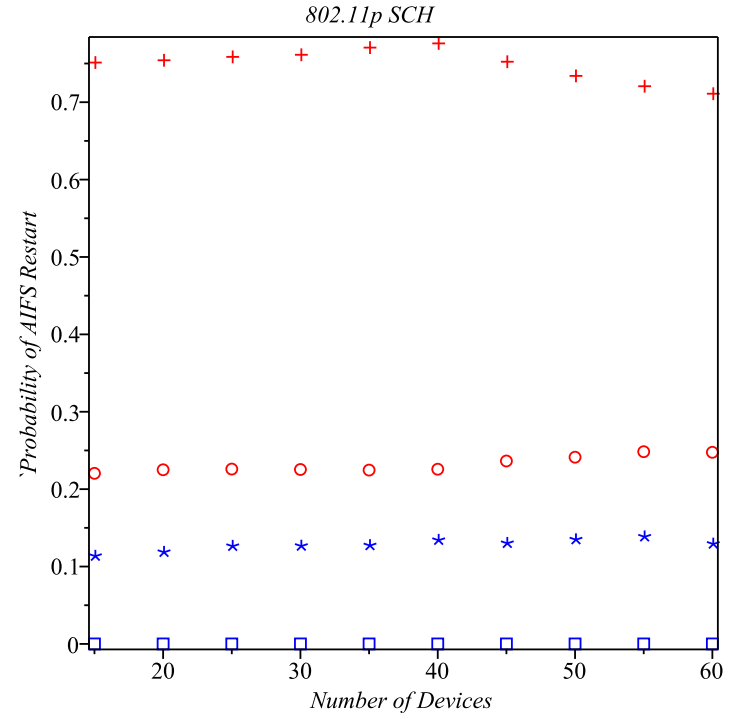

(a) AIFSN Restart

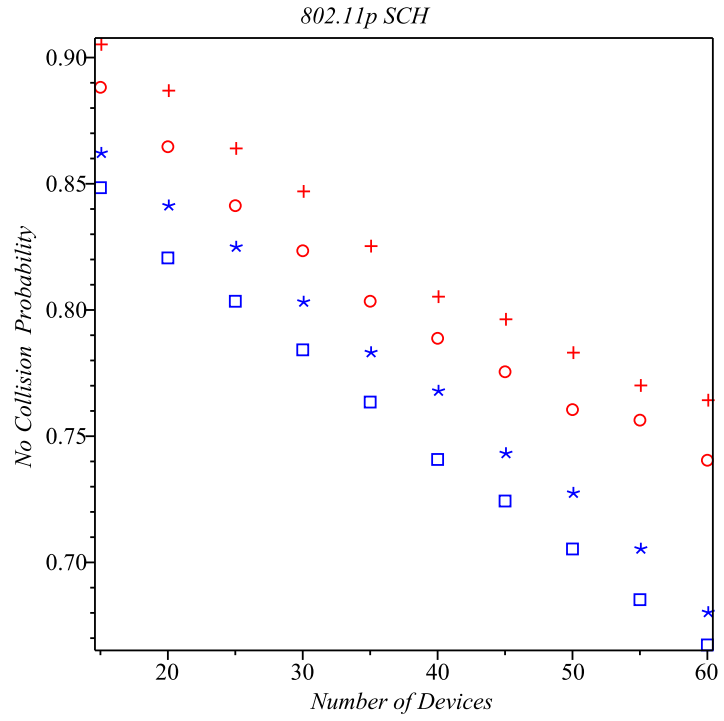

(b) No Collision Probability

Figure 4.36: AIFSN Restart Probability and No Collision Probability for 802.11p New Data Rate $\mathrm{SCH}$ for Duty Cycle equal to 0.6.

DUTY CYCLE EQUAL TO 0.6: We perform this experiment with new data rate for this duty 
cycle. The access probability increases linearly. However interesting thing to observe here is that the access probability for $\mathrm{BK}$ and $\mathrm{BE}$ flattens after there are 40 devices in the network. This is due to the saturation condition that is achieved as it is clear from the graph obtained as the high data rate for $\mathrm{BK}$ and $\mathrm{BE}$ causes more packets to be dropped than transmitted. Also the data rate for $\mathrm{VO}$ and VI was not high enough to put them in the saturation condition and they behave normally as expected. The throughput will also follow the same trend as shown by the access probability and we see that the BK and BE flattens after 40 devices. Since throughput is not increasing linearly, it affects the average backoff time and as expected it also flattens after 40 devices because less packets are transmitted than expected so average backoff time also flattens. The average backoff phase is not affected by this and it shows a linear trend. The AIFS restart probability shows that BK restarts the maximum time because of its EDCA parameters. The no collision probability for $\mathrm{BK}$ and BE starts to flatten as the number of packets that are transmitted is becoming less which means less collision for these ACs. The no collision probability would be least for this duty cycle as compared to the other ones as $\mathrm{SCH}$ has the least bandwidth in this case and highest bandwidth is given to the $\mathrm{CCH}$ so the packets that arrive in $\mathrm{SCH}$ channel when $\mathrm{CCH}$ is active queues up in the buffer and when SCH becomes active again causes packets to collide when transmitted. The VO and VI would collide the maximum in this case although they won't show the flattening which suggests that they did not go into saturation. 


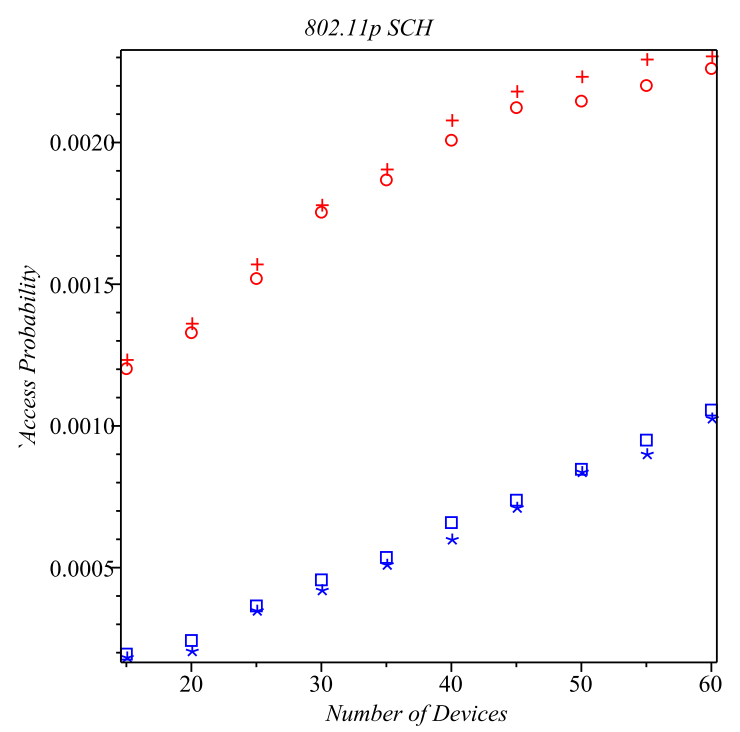

(a) Access Probability

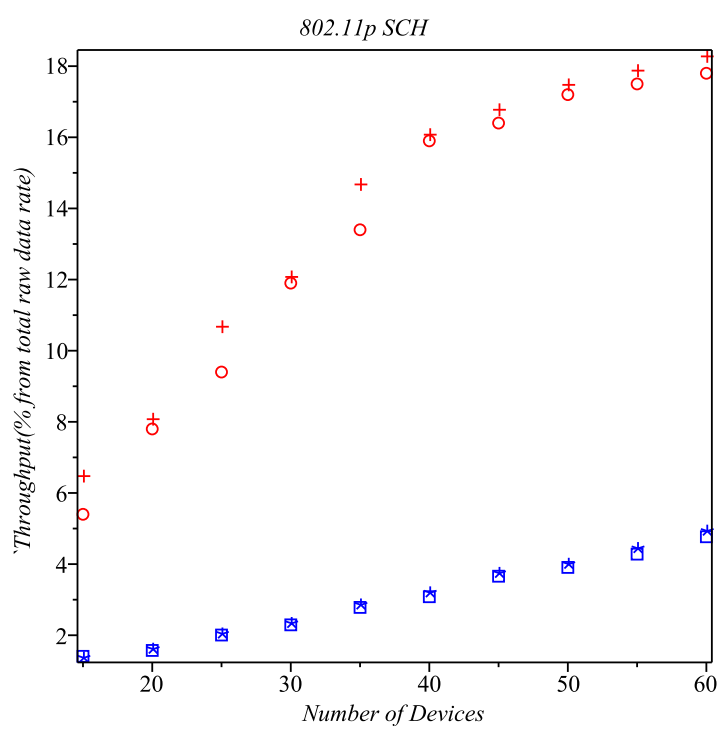

(b) Throughput

Figure 4.37: Access Probability and Throughput for $802.11 \mathrm{p} \mathrm{SCH}$ for Duty Cycle equal to 0.5 .

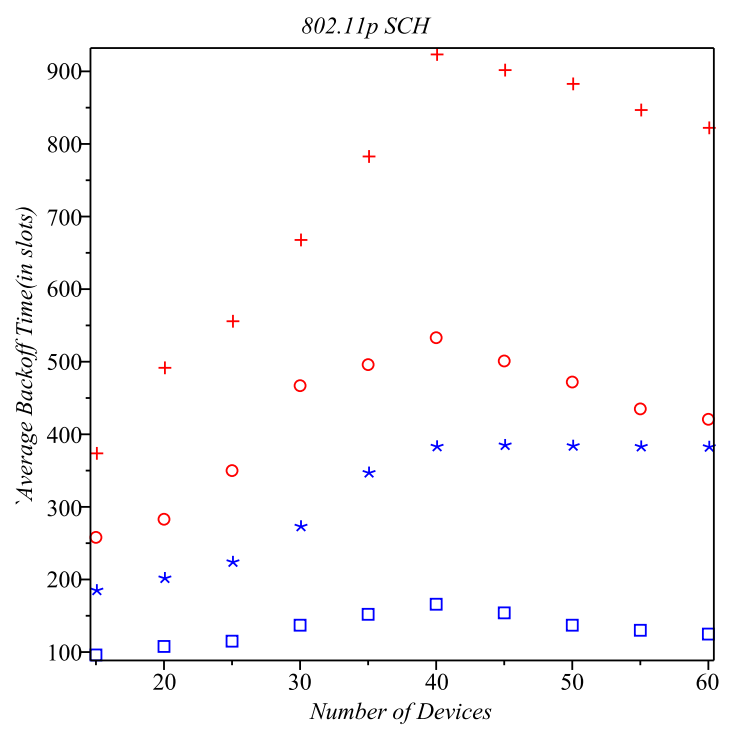

(a) Average Backoff Time

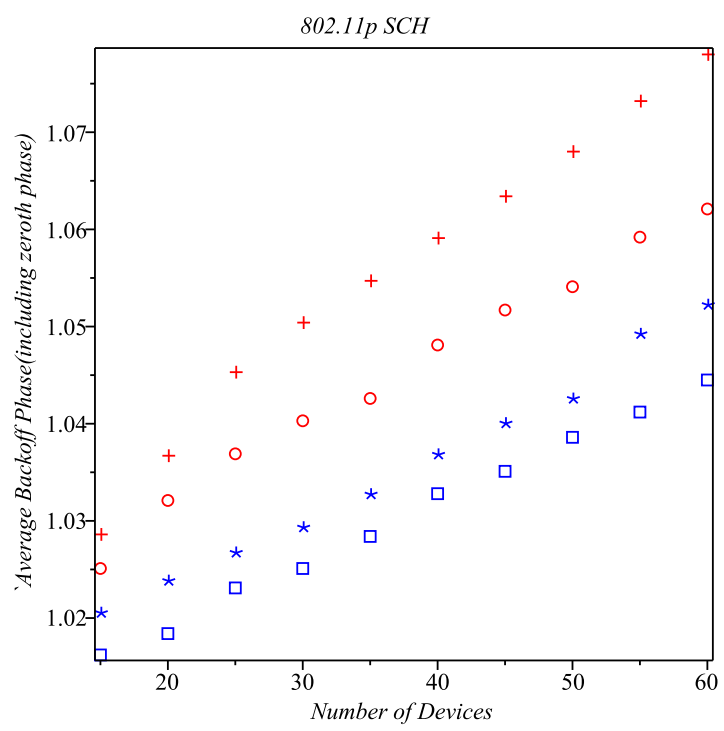

(b) Average Backoff Phase

Figure 4.38: Average Backoff Phase and Average Backoff Phase for 802.11p SCH for Duty Cycle equal to 0.5 . 


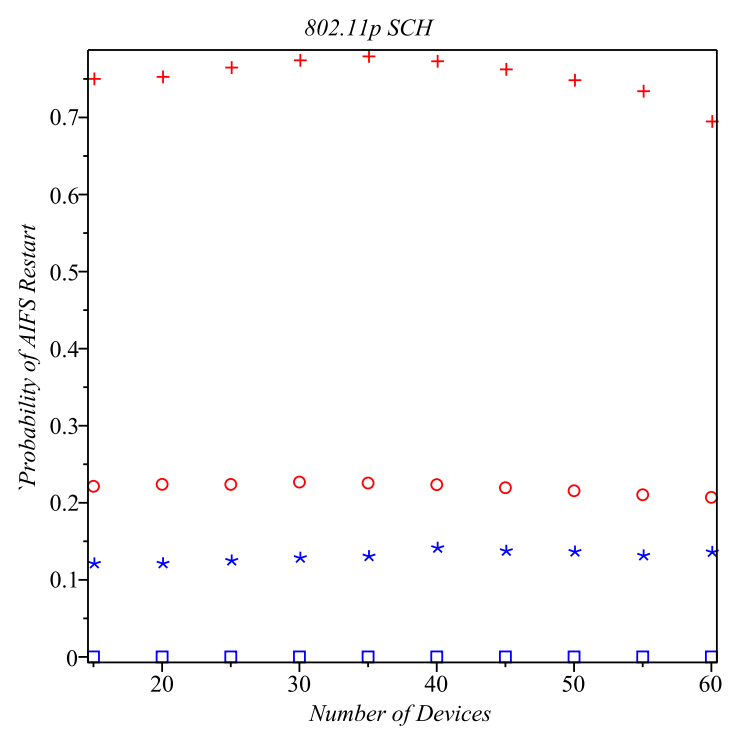

(a) AIFSN Restart

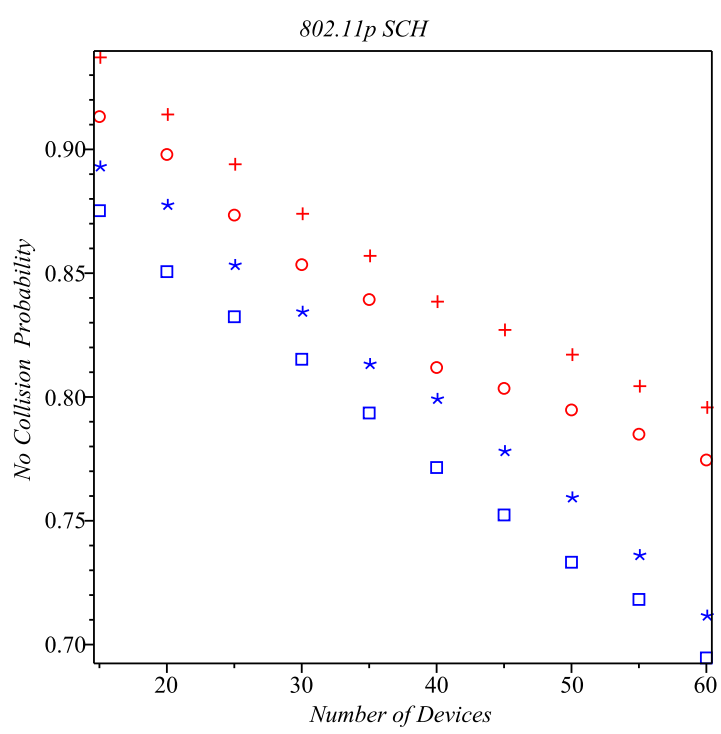

(b) No Collision Probability

Figure 4.39: AIFSN Restart Probability and No Collision Probability for 802.11p New Data Rate SCH for Duty Cycle equal to 0.5 .

DUTY CYCLE EQUAL TO 0.5: The access probability shows the linear trend before flattening when it goes into saturation similar to the 0.6 duty cycle. This in turn affects the throughput which shows the similar behaviour as obtained for the earlier duty cycle of 0.6 . The average backoff time with the increase in bandwidth decreases as compared to the duty cycle of 0.6 as ACs would complete the transmission in less time due to greater bandwidth available to SCH as compared to 0.6 but once the saturation condition is reached it starts to flatten. The average backoff phase maintains its linear trend. The AIFS restart probability is not showing any significant change and its almost in the same range as the one obtained in the previous duty cycle of 0.6 . The no collision probability increases as compared to the duty cycle of $0.6, \mathrm{BK}$ and $\mathrm{BE}$ due to saturation starts to flatten after 40 devices as compared to VO and VI which does not show this type of flattening in this scenario. 


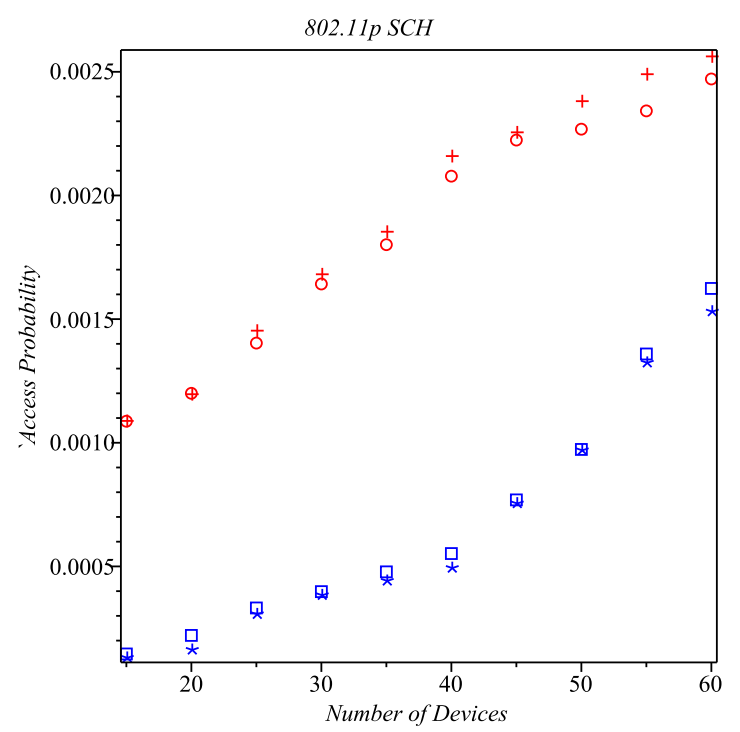

(a) Access Probability

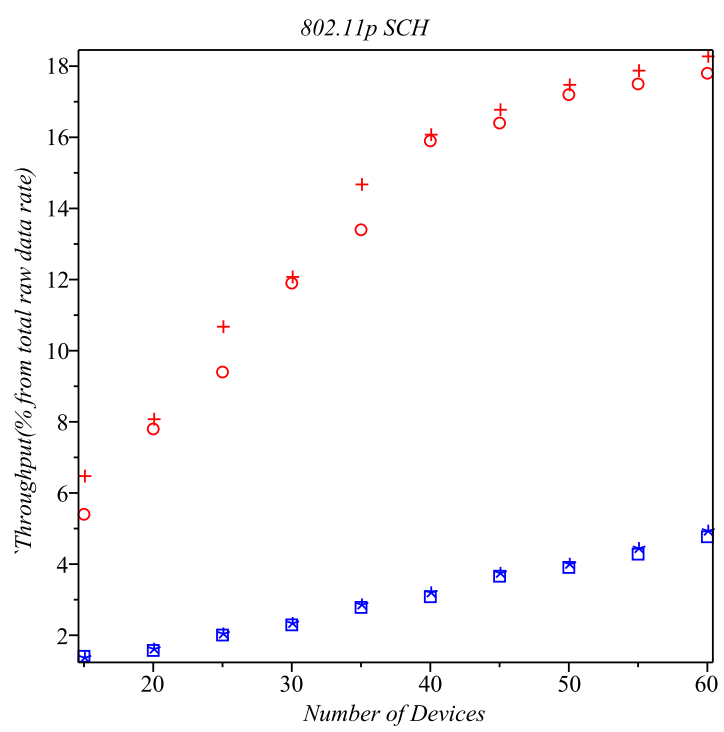

(b) Throughput

Figure 4.40: Access Probability and Throughput for $802.11 \mathrm{p} \mathrm{SCH}$ for Duty Cycle equal to 0.4.

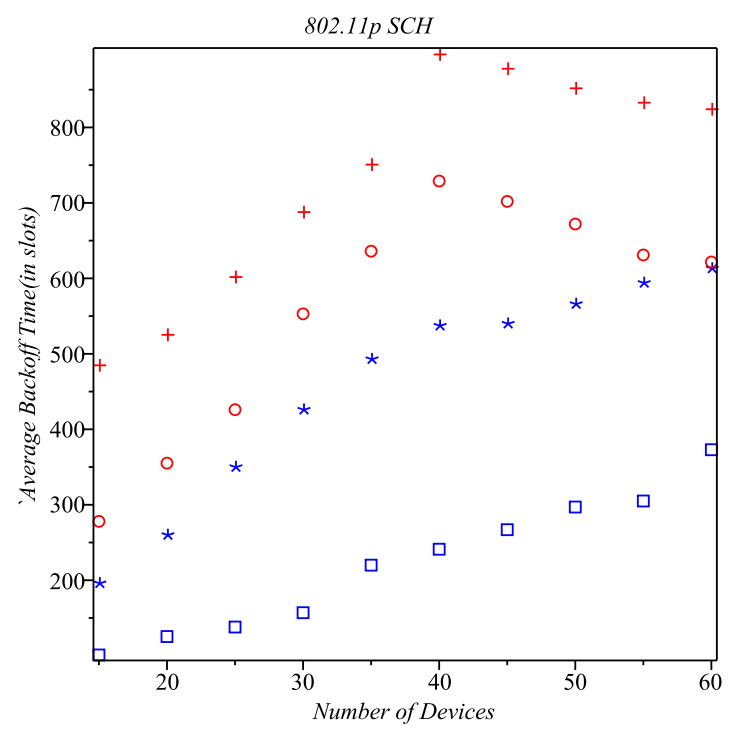

(a) Average Backoff Time

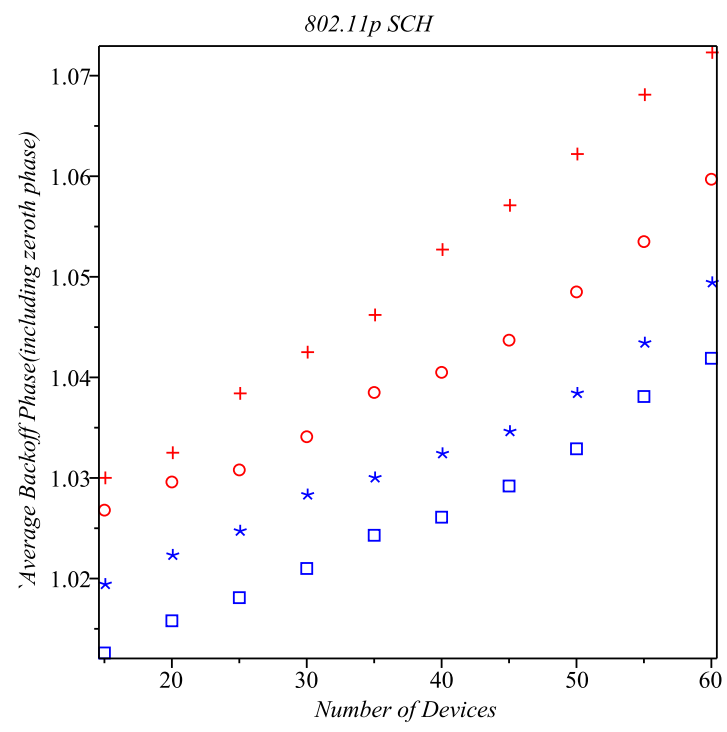

(b) Average Backoff Phase

Figure 4.41: Average Backoff Time and Average Backoff Phase for 802.11p SCH for Duty Cycle equal to 0.4 . 


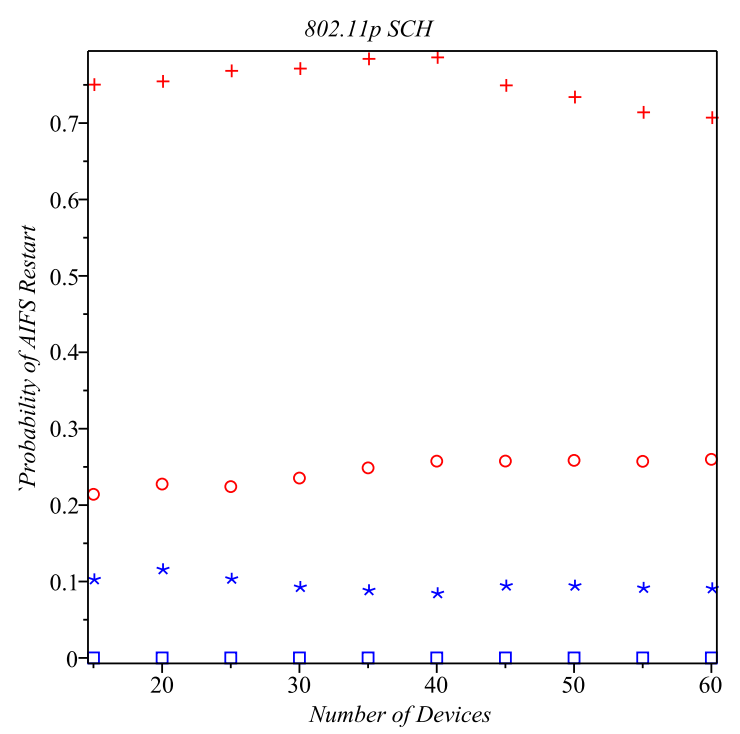

(a) AIFSN Restart

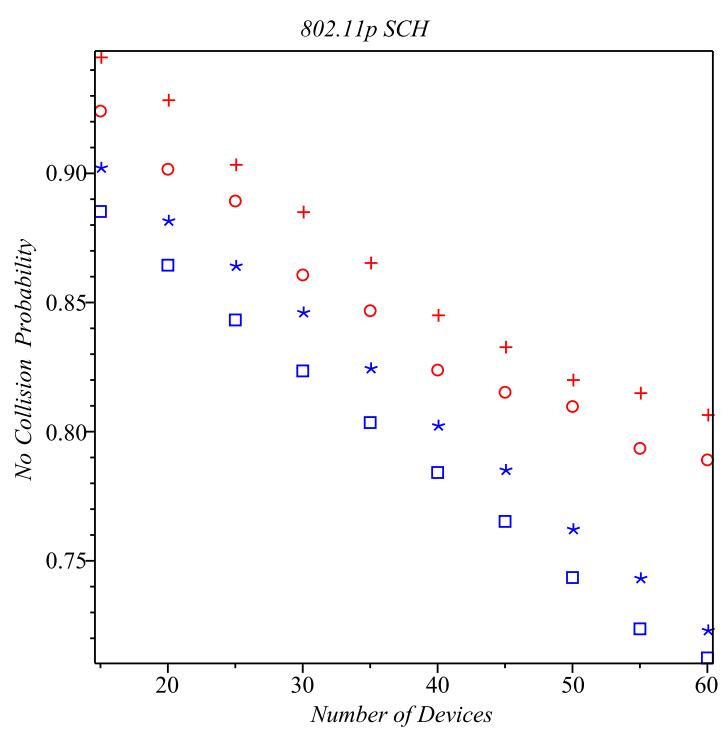

(b) No Collision Probability

Figure 4.42: AIFSN Restart Probability and No Collision Probability for 802.11p New Data Rate SCH for Duty Cycle equal to 0.4.

DUTY CYCLE EQUAL TO 0.4: The access probability shows the same behaviour of flattening as obtained in the previous duty cycles. The throughput also shows the same trend as obtained in the previous duty cycles. The average backoff value decreases further as more transmissions are completed due to increase in bandwidth with this duty cycle but BK and BE flattens once saturation condition is obtained. The average backoff phase value shows the linear trend. The AIFS value shows the same trend with only a little change in the values. The no collision probability further increases as more bandwidth is available in this duty cycle and less packets would arrive in the inactive channel period resulting in less collisions but due to saturation the $\mathrm{BK}$ and $\mathrm{BE}$ flattens whereas VO and VI behave normally as expected because they are not saturated with their no collision probability also increasing. 


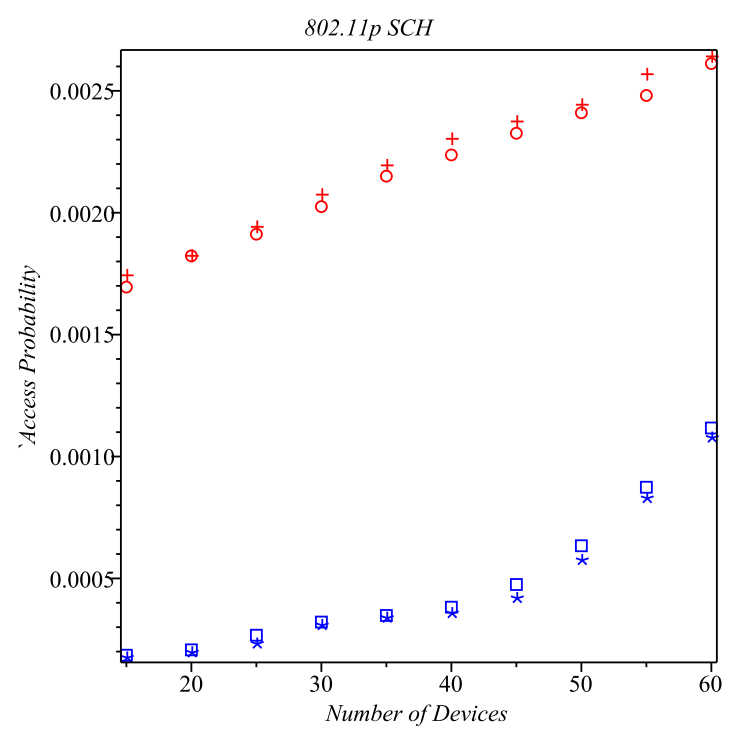

(a) Access Probability

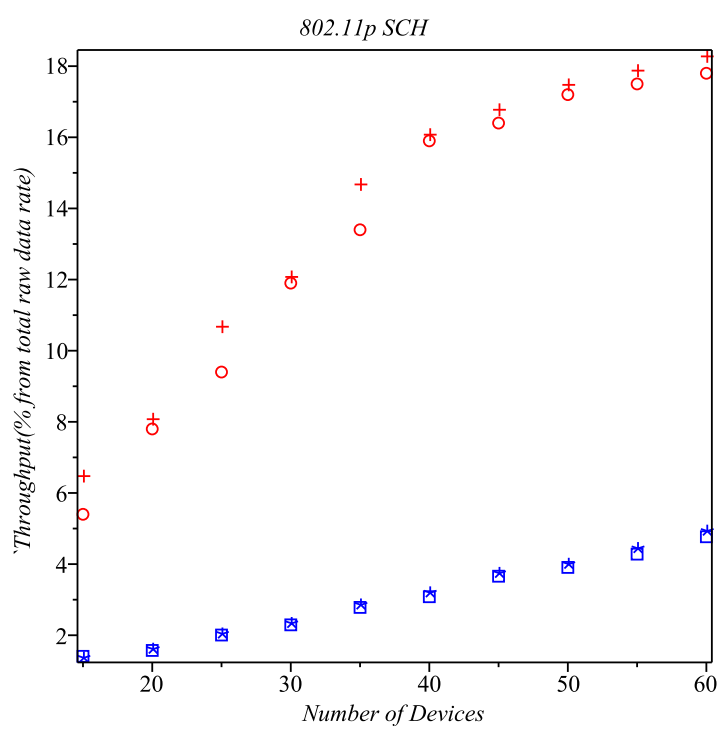

(b) Throughput

Figure 4.43: Access Probability and Throughput for $802.11 \mathrm{p} \mathrm{SCH}$ for Duty Cycle equal to 0.3 .

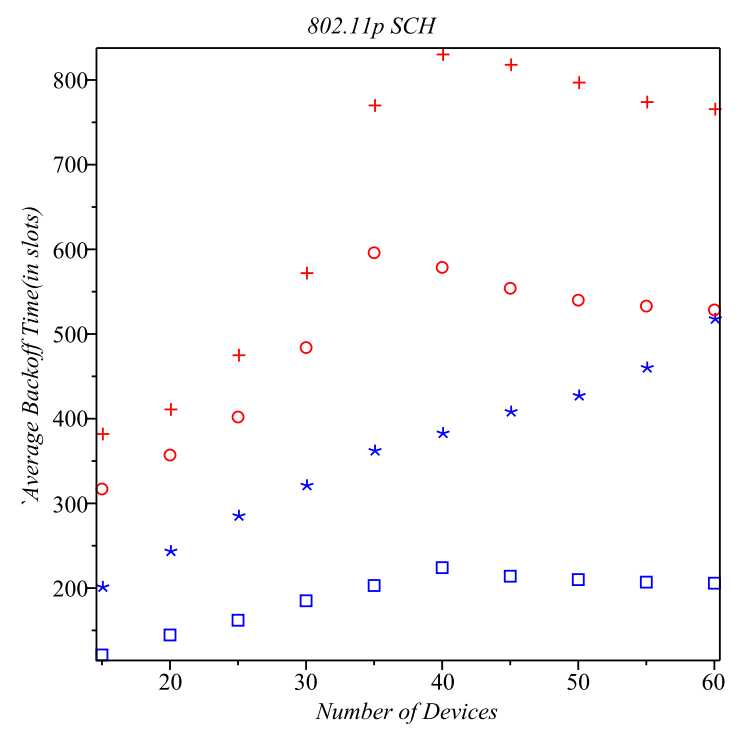

(a) Average Backoff Time

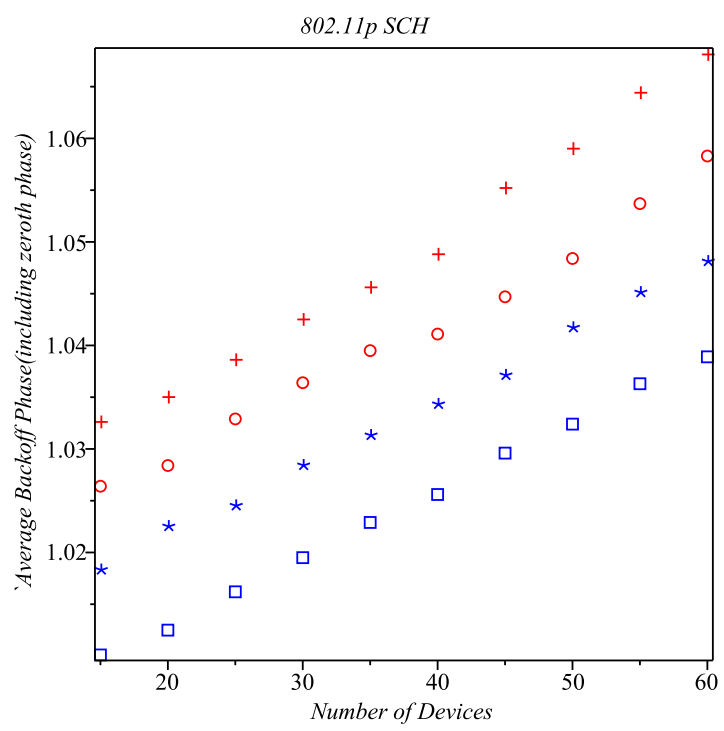

(b) Average Backoff Phase

Figure 4.44: Average Backoff Time and Average Backoff Phase for 802.11p SCH for Duty Cycle equal to 0.3 . 


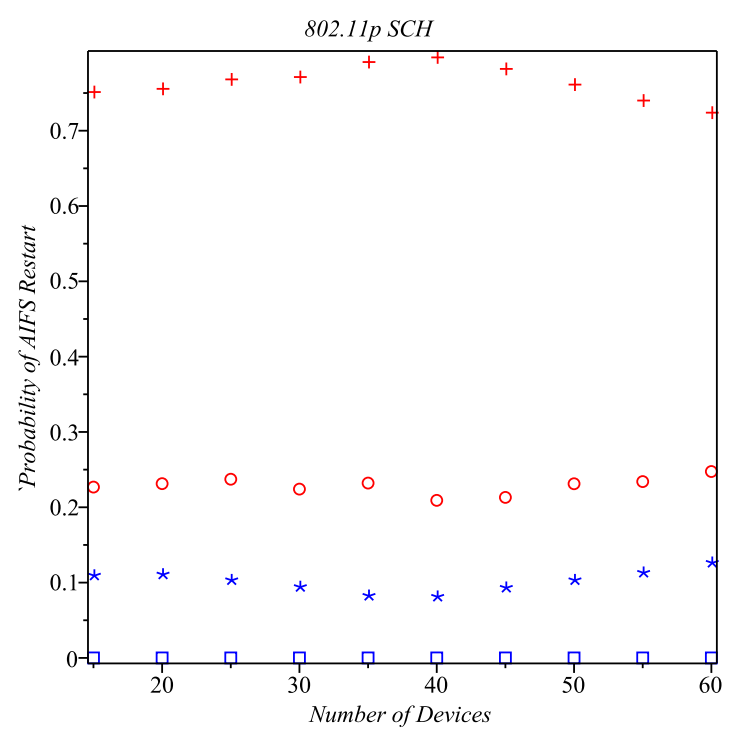

(a) AIFSN Restart

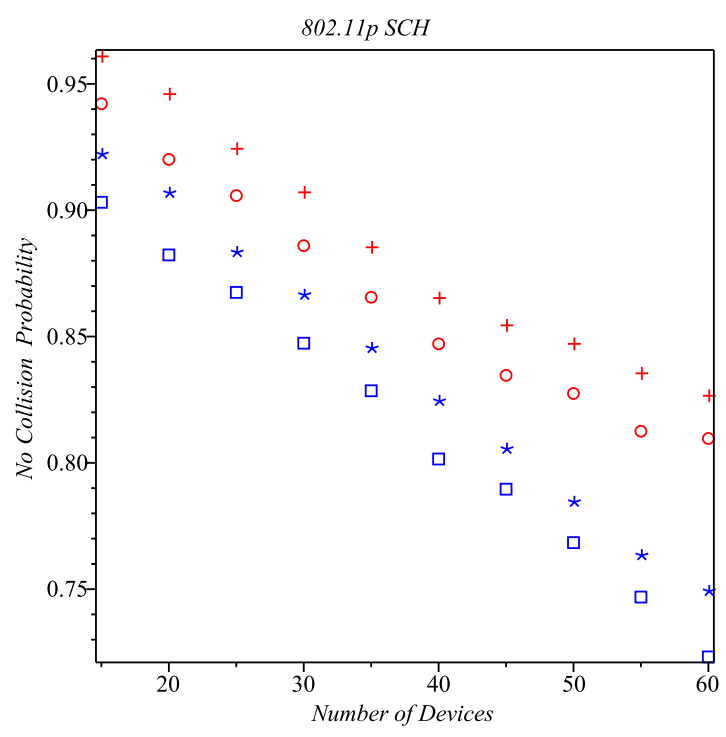

(b) No Collision Probability

Figure 4.45: AIFSN Restart Probability and No Collision Probability for 802.11p SCH for Duty Cycle equal to 0.3 .

DUTY CYCLE EQUAL TO 0.3: This duty cycle has the maximum bandwidth available as compared to all the above duty cycle that we have observed. The access probability should show linear behaviour. The throughput would be the same as obtained earlier with flattening showing the saturation. The average backoff value should be lowest as transmissions would be completed in less time but once the saturation condition is reached the backoff time should start to flatten. The average backoff phase value shows the linear trend as expected. The AIFS value does not show any significant change in the value. The no collision probability in this case is the highest as compared to all the other duty cycles observed till now with flattening of BK and BE suggesting the saturation condition. 


\section{Chapter 5}

\section{Conclusion}

We performed the experiments to evaluate the performance of the network under 802.11 DCF both in non-saturated and saturated zone. The performance of the network is optimum in the nonsaturated zone but when we have the high data rate the measured parameters are severely affected as more packets are being dropped than being transmitted which is evident from the fact that the increasing linear trend of all the parameters starts to flatten. Moreover due to the limitations of DCF that we explained earlier it is not feasible to use this technology for VANETs.

The experiments done for EDCA shows that QoS can be achieved by using 802.11e and the results were as expected with VO and VI performing the best among all the access categories. The results obtained encouraged us to perform the experiments for $802.11 \mathrm{p}$ which has the multi channel capability of data transmission.

The results obtained for $802.11 \mathrm{p}$ shows the effect of EDCA parameters (AIFSN and CW) on the performance parameters. Due to AIFSN and CW the Voice (VO) and Video (VI) perform better than Best Effort (BE) and Background (BK) on control channel. This is in accordance with the standard as VO and VI should perform the best as these are the critical and safety messages which gets priority on $\mathrm{CCH}$. However on analysis of the $\mathrm{SCH}$ it can be seen that Background (BK) and Best Effort (BE) perform better than the Voice (VO) and Video (VI) as high data rate diminishes the effect of EDCA parameters on SCH. The duty cycle along with EDCA parameters shows us that for $\mathrm{CCH}$ duty cycle of 0.6 is the best as the performance of the network is best in this duty cycle as maximum bandwidth is available to the channel during this duty cycle to complete the transmission. If we need low delay and do not care much about the performance of data on SCH 
then duty cycle of 0.6 is the best for $\mathrm{CCH}$. For $\mathrm{SCH}$ the duty cycle of 0.3 is the best as it gets the maximum bandwidth to perform the transmissions and it can also use $\mathrm{CCH}$ to receive the safety messages without its performance being affected too much. However with high loads the performance starts to degrade on $\mathrm{SCH}$ so a very careful selection of the data rate has to be done so that the performance of the network remains optimum. 


\section{Abbreviations}

\begin{tabular}{|c|c|}
\hline $\mathrm{AC}$ & Access Categories \\
\hline $\mathrm{ACK}$ & Acknowledgement \\
\hline AIFS & Arbitary Interframe Space \\
\hline $\mathrm{BE}$ & Best Effort \\
\hline BK & Background \\
\hline BSS & Basic Service Set \\
\hline $\mathrm{CCH}$ & Control Channel \\
\hline CSMA/CA & Carrier Sense Multiple Access with Collision Avoidance \\
\hline CTS & Clear-To-Send \\
\hline CWmin & Contention Window Minimum \\
\hline CWmax & Contention Window Maximum \\
\hline $\mathrm{DCF}$ & Distributed Coordination Function \\
\hline DSRC & Dedicated Short Range Communication \\
\hline EDCA & Enhanced Distributed Channel Access \\
\hline ESS & Extended Service Set \\
\hline HCCA & Hybrid Controlled Channel Access \\
\hline IEEE & Institute of Electrical and Electronics Engineers \\
\hline IFS & Interframe Spaces \\
\hline ISM & Industrial, Scientific and Medical \\
\hline MAC & Medium Access Control \\
\hline MANETs & Mobile Adhoc Networks \\
\hline
\end{tabular}




\begin{tabular}{ll} 
NAV & Network Allocation Vector \\
PCF & Point Coordinated Function \\
RBC & Quality of Service \\
RTS & Random Backoff Countdown \\
SCH & Service Channel \\
SIFS & Short Interframe Space \\
SO & Superframe Order \\
STA & Station \\
TXOP & Transmission Opportunity \\
UTC & Coordinated Universal Time \\
VANETs & Vehicular Adhoc Networks \\
VO & Voice \\
VI & Video \\
WLAN & Wireless LAN \\
\hline
\end{tabular}




\section{Bibliography}

[1] Draft Amendment for Wireless Access in Vehicular Environments(WAVE), IEEE Std.802.11p D3.0, 2007

[2] Rsoft Design Group. San Jose, CA, 2003. Artifex v.4.4.2.

[3] Maple 13, Maplesoft, Inc, Waterloo, ON, Canada, 2009

[4] http://www.globalissues.org/news/2011/05/31/9877

[5] L. Kleinrock and F. Tobagi, Packet switching in radio channels, Part II, The hidden terminal problem in carrier sense multiple access and the busy tone solution, IEEE Trans. Commun., vol. COM-23, no. 12, pp. 1417-1433, Dec. 1975

[6] H. S. Chhaya and S. Gupta, Performance modeling of asynchronous data transfer methods of IEEE 802.11 MAC protocol, Wireless Networks, vol. 3, pp. 217-234, 1997

[7] K. C. Huang and K. C. Chen, Interference analysis of nonpersistent CSMA with hidden terminals in multicell wireless data networks, in Proc. IEEE PIMRC, Toronto, Canada, Sept. 1995, pp. 907-91

[8] G.Bianchi, Performance Analysis of the IEEE 802.11 Distributed Coordination Function, IEEE Journal on Selected Area in Communications, vol.18, no.3, pp. 535-547, 2000

[9] H. Wu, Y. Peng, K. Long, J. Ma, Performance of Reliable Transport Protocol over IEEE 802.11 Wireless LAN: Analysis and Enhancement, Proc. of IEEE INFOCOM, vol.2, pp. 599-607, 2002

[10] G. Bianchi, IEEE 802.11Saturation throughput analysis, IEEE Commun. Lett., vol. 2, pp. 318-320, Dec. 1998. 
[11] B. P. Crow, Performance evaluation of the IEEE 802.11 Wireless Local Area Network Protocol, M.S. thesis, Dept. Electrical and Computer Eng., Univ. Arizona, Tucson, AZ, 1996.

[12] J. Weinmiller, M. Schlager, A. Festag, and A. Wolisz, Performance study of access control in wireless LANs IEEE 802.11 DFWMAC and ETSI RES 10 HIPERLAN, Mobile Networks and Applicat., vol. 2, pp. 55-67, 1997.

[13] K.P.Manikandan, Dr.R.Satyaprasad, Dr.Rajasekhararao, "A Comprehensive Review on Ameliorate the Quality of Services over IEEE Standard 802.11 Based MAC Layer in Mobile Ad hoc Network",Vol. 2, No. 2, April 2011

[14] IEEE. Standard for part 11: Wireless lan medium access control (MAC) and physical layer (PHY) specifications. IEEE Std 802.11, IEEE, New York, NY, 2007

[15] IEEE 802.11e/D13.0, Draft Supplement to Part 11: Wireless LAN Medium Access Control (MAC) and Physical Layer (PHY) Specifications: Medium Access Control (MAC) Quality of Service (QoS) Enhancements. January 2005.

[16] P. E. Engelstad and O. N. sterb, Analysis of the total delay of IEEE 802.11e EDCA and 802.11 DCF, in Proceedings of the IEEE International Conference on Communications (ICC '06), pp. 552-559, Istanbul, Turkey, July 2006.

[17] W. Zhang, J. Sun, J. Liu, and H. B. Zhang, Performance analysis of IEEE 802.11e EDCA in wireless LANs, Journal of Zhejiang University, vol. 8, no. 1, pp. 18-23, 2007.

[18] Y. Xiao, Performance analysis of IEEE 802.11e EDCF under saturation condition, in Proceedings of the IEEE International Conference on Communications (ICC '04), pp. 170-174, Chicago, Ill, USA, June 2004.

[19] J. Hui and M. Devetsikiotis, A unified model for the performance analysis of IEEE 802.11e EDCA, IEEE Transactions on Communications, vol. 53, no. 9, pp. 1498-1510, 2005. 
[20] Z. N. Kong, D. H. K. Tsang, B. Bensaou, and D. Gao, Performance analysis of IEEE 802.11e contention-based channel access, IEEE Journal on Selected Areas in Communications, vol. 22, no. 10 , pp. $2095-2106,2004$

[21] Y. Xiao, Performance analysis of priority schemes for IEEE 802.11 and IEEE 802.1 le wireless LANs, IEEE Transactions on Wireless Communications, vol. 4, no. 4, pp. 1506-1515, 2005.

[22] Y. Lin and V. W. S. Wong, Saturation throughput of IEEE 802.11e EDCA based on mean value analysis, in Proceedings of the IEEE Wireless Communications and Networking Conference (WCNC '06), pp. 475-480, Las Vegas, Nev, USA, April 2006.

[23] I. Inan, F. Keceli, and E. Ayanoglu, Modeling the 802.11e enhanced distributed channel access function, in Proceedings of the 50th Annual IEEE Global Telecommunications Conference (GLOBECOM ’07), pp. 2546-2551, Washington, DC, USA, Nov 2007.

[24] A. Vinel, Y. Koucheryavy, S. Andreev, and D. Staehle, Estimation of a successful beacon reception probability in vehicular ad-hoc networks, in Proc. IWCMC: Connecting the World Wirelessly, Jun. 2009, pp. 416-420

[25] L. Cheng, B. E. Henty, D. D. Stancil, F. Bai, and P. Mudalige, Mobile vehicle-to-vehicle narrow-band channel measurement and characterization of the $5.9 \mathrm{GHz}$ dedicated short range communication (DSRC) frequency band, IEEE J. Sel. Areas Commun., vol. 25, no. 8, pp. 1501-1516, Oct. 2007

[26] Q. Xu, T. Mak, J. Ko, and R. Sengupta, Medium access control protocol design for vehiclevehicle safety messages, IEEE Trans. Veh. Technol., vol. 56, no. 2, pp. 499-518, Mar. 2007

[27] Y. Zang et al., Towards Broadband Vehicular Ad-Hoc Networks the Vehicular Mesh Network (VMESH) MAC Protocol, IEEE WCNC, 2007. 
[28] S.Y. Wang et al., Improving the Channel Utilization of IEEE 802.11p/ 1609 Networks, IEEE WCNC, 2009.

[29] K. Liu, J. Guo, N. Lu, F. Lu, RAMC: A RSU-assisted multi-channel coordination MAC protocol for VANET, in: NiVi Workshop co-located with IEEE, Globecom, 2009.

[30] S. Ou, K. Yang, H. Chen, A. Galis, A selective downlink scheduling algorithm to enhance quality of VOD services for WAVE networks, EURASIP Journal on Wireless Communications and Networking (2009), 112.

[31] Jelena Misic, Ghada Badawy,and Vojislav Misic, "Trade Off Issues for CCH/SCH Duty Cycle for IEEE 802.11p Single Channel Devices"”, Proceedings of 2010 IEEE Globecom conference (Globecom’10), December 6-10, 2010.

[32] N. Balan and J. Guo, Increasing broadcast reliability in vehicular ad hoc Networks, in Proc. ACM VANET, Los Angeles, CA, USA, 2006

[33] IEEE Trial-Use Standard for Wireless Access in Vehicular Environments (WAVE)MultiChannel Operation, IEEE Std. 1609.4-2007, 2007.

[34] William Stallings. Data and computer communications. Prentice Hall.5th Edition. 1996

[35] S Gillani, I Khan, S Qureshi, A Qayyum- Vehicular Ad Hoc Network (VANET): Enabling Secure and Efficient Transportation, 2009

[36] IEEE Trial-Use Standard for Wireless Access in Vehicular Environments (WAVE)MultiChannel Operation, IEEE Std. 1609.1-2007, 2007.

[37] IEEE Trial-Use Standard for Wireless Access in Vehicular Environments (WAVE)Security Services for Applications and Management Messages, IEEE Std. 1609.2-2007, 2007. 
[38] IEEE Trial-Use Standard for Wireless Access in Vehicular Environments (WAVE)Networking Services, IEEE Std. 1609.3-2007, 2007.

[39] IEEE Trial-Use Standard for Wireless Access in Vehicular Environments (WAVE)MultiChannel Operation, IEEE Std. 1609.4-2007, 2007.

[40] P. Brenner. A Technical Tutorial on the IEEE 802.11 Protocol. BreezeCom Wireless Communications, 1992. 